\title{
ON SUFFICIENT CONDITIONS FOR HAMILTONICITY IN DENSE GRAPHS
}

\author{
RICHARD LANG AND NICOLÁS SANHUEZA-MATAMALA
}

\begin{abstract}
We study structural conditions in dense graphs that guarantee the existence of vertex-spanning substructures such as Hamilton cycles. It is easy to see that every Hamiltonian graph is connected, has a perfect fractional matching and, excluding the bipartite case, contains an odd cycle. Our main result in turn states that any large enough graph that robustly satisfies these properties must already be Hamiltonian. Moreover, the same holds for embedding powers of cycles and graphs of sublinear bandwidth subject to natural generalisations of connectivity, matchings and odd cycles.

This solves the embedding problem that underlies multiple lines of research on sufficient conditions for Hamiltonicity in dense graphs. As applications, we recover and establish Bandwidth Theorems in a variety of settings including Ore-type degree conditions, Pósatype degree conditions, deficiency-type conditions, locally dense and inseparable graphs, multipartite graphs as well as robust expanders.
\end{abstract}

\section{INTRODUCTION}

An old question in discrete mathematics is whether a given graph contains certain vertexspanning substructures, such as a Hamilton cycle. Since the corresponding decision problems are often computationally intractable, we do not expect to find 'simple' characterisations of the graphs that contain a particular spanning structure. The extremal approach to these questions has therefore focused on easily-verifiable sufficient conditions. A classic example in this direction is Dirac's theorem [20], which states that every graph on $n \geqslant 3$ vertices and minimum degree at least $n / 2$ contains a Hamilton cycle. Since its inception, Dirac's theorem has been extended in numerous many ways by replacing the assumptions on the host graph and the conditions on the guest graph [31, 49]. Here, we propose a general framework to approach these problems in dense graphs, and apply it to prove several new results.

One natural way to extend Dirac's theorem is to weaken the assumptions on the host graph. Simple constructions show that a minimum degree of $n / 2$ is best-possible. However, as it turns out, not all of the vertices need to have this degree in order to ensure that the graph is Hamiltonian. A well-known theorem of Ore [63] states that a graph on $n$ vertices contains a Hamilton cycle if $\operatorname{deg}(u)+\operatorname{deg}(v) \geqslant n$ for all pairs of non-adjacent vertices $u$ and $v$. Pósa [64] extended this by showing that a graph on $n$ vertices contains a Hamilton cycle provided that its degree sequence $d_{1} \leqslant \ldots \leqslant d_{n}$ satisfies $d_{i} \geqslant i+1$ for all $i \leqslant n / 2 .{ }^{1}$ A decade later, Chvátal [16] gave a complete characterisation of the degree sequences that guarantee Hamiltonicity. Similar results have been obtained in the setting of bipartite host graphs [61]. More recently, structural assumptions such as local density together with inseparability, deficiency conditions and robust expansion have been investigated, which we will discuss in more detail below.

Another possibility to generalise Dirac's theorem consists in embedding structurally more complex guest graphs, such as clique factors and powers of cycles. A $k$-clique factor in a graph $G$ consists of pairwise disjoint $k$-cliques (complete graphs on $k$ vertices) that cover all vertices of $G$. It was conjectured by Erdős and proved by Hajnal and Szemerédi [32] that graphs on $n$ vertices and minimum degree at least $(k-1) n / k$ have a $k$-clique factor,

Date: 29th October 2021.

The research leading to these results was partly supported by the Czech Science Foundation, grant number GA19-08740S with institutional support RVO: 67985807 (N. Sanhueza-Matamala) and also by the Deutsche Forschungsgemeinschaft (DFG, German Research Foundation) - 450397222 (R. Lang).

${ }^{1}$ Indeed, a quick exercise shows that every graph satisfying Ore's conditions also satisfies Pósa's conditions. 
assuming the obvious necessary condition that $k$ divides $n$. Similarly, the notion of cycles can be generalised in terms of their powers. The $k$ th power (or square when $k=2$ ) of a graph $G$ is obtained from $G$ by joining any two vertices of distance at most $k$. Pósa (for $k=3$ ) and Seymour (for $k \geqslant 3$ ) conjectured that any graph on $n$ vertices with minimum degree at least $(k-1) n / k$ contains the $(k-1)$ th power of a Hamilton cycle. This was confirmed by Komlós, Sárközy and Szemerédi [44] for sufficiently large $n$. While powers of cycles might appear to be a somewhat particular class of graphs, their embedding turned out to be an important milestone with regards to embedding the much richer class of $k$-colourable graphs with bounded degree and sublinear bandwidth (as defined below) under essentially the same minimum degree conditions necessary for $k$-clique factors. This last result is known as the Bandwidth Theorem and was proved by Böttcher, Schacht and Taraz [8].

In the recent years there has been a surge of activity in the study of whether and when the above described assumptions on the host graph allow to embed increasingly complex guest graphs (as surveyed below). Many of these results are proved using similar embedding techniques, but differ in their structural analysis. One might therefore wonder whether there is a common structural base that 'sits between' all of these assumptions and (variations of) Hamiltonicity. For the (ordinary) Hamilton cycles, this was done by Kühn, Osthus and Treglown [52] by means of the notion of robust expanders. Ebsen, Maesaka, Reiher, Schacht and Schülke [22] raised the question of generalising the concept of robust expanders to handle powers of cycles and other $k$-colourable graphs.

In this paper, we introduce the notion of Hamilton frameworks (Definition 3.1), which comprises the characteristic properties of powers of Hamilton cycles, while remaining computationally tractable. Our main results (Theorems 3.4 and 3.5) state that graphs that have a robust Hamilton framework are (in a strong sense) Hamiltonian and admit the corresponding powers of cycles. As an application we can easily recover the above mentioned contributions and also prove several new results, including multiple conjectures.

\section{Applications}

To formulate extensions of the Bandwidth Theorem, we introduce some further notation. A graph $H$ admits an ordering with bandwidth at most $b$ if the vertices of $H$ can be labelled with $1, \ldots, n$ such that $|i-j| \leqslant b$ for all edges $i j$. We abbreviate this by $\operatorname{bw}(H) \leqslant b$. The chromatic number and maximum degree of $H$ are denoted by $\chi(H)$ and $\Delta(H)$, respectively.

Definition $2.1((\beta, \Delta, k)$-Hamiltonian). A graph $G$ on $n$ vertices is $(\beta, \Delta, k)$-Hamiltonian if $G$ contains every graph $H$ on $n$ vertices with $\chi(H) \leqslant k, \Delta(H) \leqslant \Delta$ and $\operatorname{bw}(H) \leqslant \beta n$.

Note that, for $\beta>0$ and $n$ large enough, this includes $(k-1)$ th powers of cycles $H$ on $n$ vertices when $n$ is divisible by $k$, since $H$ is $k$-colourable in this case.

Following Allen, Böttcher, Ehrenmüller and Taraz [1], we also consider $(k+1)$-coloured graphs where one colour is used only sparingly as follows. A $\beta$-block of $\{1, \ldots, n\}$ is an interval of the type $\{(i-1)\lceil\beta n\rceil+1, \ldots, i\lceil\beta n\rceil\}$ for some $1 \leqslant i \leqslant \beta^{-1}$. A $(k+1)$-colouring $\chi:\{1, \ldots, n\} \rightarrow\{0,1, \ldots, k\}$ is said to be $(z, \beta)$-zero-free if, among every $z$ consecutive $\beta$-blocks at most one of them uses colour 0 .

Definition $2.2((z, \beta, \Delta, k)$-Hamiltonian). A graph $G$ on $n$ vertices is $(z, \beta, \Delta, k)$-Hamiltonian if $G$ contains all graphs $H$ on $n$ vertices with $\Delta(H) \leqslant \Delta$ which admit an ordering with bandwidth at most $\beta n$ and a $(z, \beta)$-zero-free $(k+1)$-colouring.

We remark that this last definition covers $(k-1)$ th powers of cycles $H$ (of any order), when $\beta>0, z \geqslant 1 / \beta$ and $n$ is large enough. The aforementioned Bandwidth Theorem by Böttcher, Schacht and Taraz, in particular, implies that for any $\mu>0, k \geqslant 2, \Delta>0$, there exists $z, \beta>0$ such that every sufficiently large graph $G$ on $n$ vertices with $\delta(G) \geqslant((k-1) / k+\mu) n$ is $(z, \beta, \Delta, k)$-Hamiltonian.

In the following, we present a series of consequences of our main results (Theorems 3.4 and 3.5). We remark that each of these applications benefits from already established insights on clique factors and, in case of uniformly dense and inseparable graphs, the connectivity 
structure, which are used as a black boxes. The proofs of these results can be found in Section 5 .

2.1. Ore-type conditions. Motivated by the Hajnal-Szemerédi Theorem, Kierstead and Kostochka [40] investigated optimal Ore-type conditions which ensure the existence of clique factors and proved the following result.

Theorem 2.3 (Clique factors under Ore-type conditions). For $n$ divisible by $k$, let $G$ be $a$ graph on $n$ vertices with $\operatorname{deg}(x)+\operatorname{deg}(y) \geqslant 2 \frac{k-1}{k} n-1$ for all $x y \notin E(G)$. Then $G$ contains a $k$-clique factor.

Note that the result is tight, as witnessed, for instance, by slightly imbalanced complete $k$ partite graphs. As an extension of this, Kühn, Osthus and Treglown [51] showed an Ore-type result for general factors (not just cliques). For sufficiently large graphs, Châu [13] proved a generalisation of Ore's theorem for squares of Hamilton cycles, and also conjectured generalisations of this for all $k \geqslant 3$. For $k=2$, Knox and Treglown [41] were able to strengthen Ore's theorem to $(\beta, \Delta, 2)$-Hamiltonian graphs. They also conjectured corresponding extensions for all $k \geqslant 3$. This was confirmed for $k=3$ by Böttcher and Müller [6]. The following result proves these conjectures in a strong sense for all $k \geqslant 2$.

Theorem 2.4 (Bandwidth theorem under Ore-type conditions). For $k, \Delta \in \mathbb{N}$ and $\mu>0$, there are $z, \beta>0$ and $n_{0} \in \mathbb{N}$ with the following property. Let $G$ be a graph on $n \geqslant n_{0}$ vertices with $\operatorname{deg}(x)+\operatorname{deg}(y) \geqslant 2 \frac{k-1}{k} n+\mu n$ for all $x y \notin E(G)$. Then $G$ is $(z, \beta, \Delta, k)$-Hamiltonian.

2.2. Pósa-type conditions. Balogh, Kostochka and Treglown [3, 4] studied degree conditions that guarantee the existence of clique factors and powers of Hamilton cycles. Treglown [71] proved the following Pósa-type result for clique factors.

Theorem 2.5 (Clique factors under Pósa-type conditions). For $k \in \mathbb{N}$ and $\mu>0$, there is an $n_{0} \in \mathbb{N}$ with the following property. Let $G$ be a graph with degree sequence $d_{1}, \ldots, d_{n}$ where $n \geqslant n_{0}$ is divisible by $k$. Suppose that $d_{i} \geqslant \frac{k-2}{k} n+i+\mu n$ for every $i \leqslant n / k$. Then $G$ contains a $k$-clique factor.

We remark that the degree conditions in Theorem 2.5 are best possible apart from the term $\mu n$. This result further was extended by Hyde, Liu and Treglown [34] and Hyde and Treglown [35] to degree conditions which ensure factors and partial factors of arbitrary graphs (not just cliques).

Balogh, Kostochka and Treglown [3] asked whether one could improve the degree sequences which ensure the existence of $(k-1)$ th power of a Hamilton cycle by allowing a non-negligible number of vertices to have degree less than $(k-1) n / k$. Staden and Treglown [68] answered this question for $k=3$, showing that the conditions of Theorem 2.5 also imply the existence of a squared Hamilton cycle and conjectured extensions of this to all $k \geqslant 4$.

We prove this conjecture in the (more general) bandwidth setting. Here, Knox and Treglown [41] had previously proved a Bandwidth Theorem for degree sequences for bipartite graphs, that is when $k=2$. Staden and Treglown [68] conjectured such a result could be true for $k=3$, and Treglown [72] extended the conjecture to all $k \geqslant 4$, which we hence confirm as well.

Theorem 2.6 (Bandwidth theorem under Pósa-type conditions). For $k \geqslant 2, \Delta \in \mathbb{N}$ and $\mu>0$, there are $z, \beta>0$ and $n_{0} \in \mathbb{N}$ with the following property. Let $G$ be a graph with degree sequence $d_{1}, \ldots, d_{n}$ such that $d_{i} \geqslant \frac{k-2}{k} n+i+\mu n$ for every $i \leqslant n / k$. Then $G$ is $(z, \beta, \Delta, k)$-Hamiltonian.

We remark that the degree conditions are essentially tight: by adapting examples of Balogh, Kostochka and Treglown [3], we show Theorem 2.6 becomes false if $\mu n$ is replaced by $o\left(n^{1 / 2}\right)$ (see Appendix A.1). Moreover, unlike the case $k=2$, the conditions of Theorem 2.6 and of Theorem 2.4 do not imply each other whenever $k \geqslant 3$, as witnessed by further constructions (see Appendix A.2). 
2.3. Locally dense and inseparable graphs. Another type of sufficient conditions for Hamiltonicity involves the notion of locally dense graphs. For a set of vertices $U$ in a graph $G$, we denote by $e(U)$ the number of edges of $G$ which are contained in $U$. For $\varrho, d>0$, we say that a graph $G$ on $n$ vertices is $(\varrho, d)$-dense if $e(U) \geqslant d|U|^{2} / 2-\varrho n^{2}$ for every $U \subseteq V(G)$. It was shown by Staden and Treglown [69] that for all $k, \Delta$, and a suitable choice of parameters, locally dense graphs of minimum degree $(1 / 2+\mu)$ are $(\beta, \Delta, k)$-Hamiltonian. Note that in particular, the degree condition does not any longer depend on $k$.

This result was further generalised by Ebsen, Maesaka, Reiher, Schacht and Schülke [22] by replacing the minimum degree with a condition of inseparability. For $\mu>0$, we say a graph $G$ is $\mu$-inseparable if $e(X, Y) \geqslant \mu|X||Y|$ for every partition $\{X, Y\}$ of the vertex set of $G$. Ebsen et al. proved Hamiltonicity for powers of cycles in locally dense and inseparable graphs, and extended this to the bandwidth setting. Our framework allows us to recover this result.

Theorem 2.7 (Bandwidth Theorem for uniformly dense and inseparable graphs, [22]). For $k, \Delta \in \mathbb{N}$ and $\mu>0$, there are $\beta, \varrho, d>0$ and $n_{0} \in \mathbb{N}$ with the following property. Let $G$ be $a$ $(\varrho, d)$-dense and $\mu$-inseparable graph on $n \geqslant n_{0}$ vertices. Then $G$ is $(\beta, \Delta, k)$-Hamiltonian.

2.4. Deficiency-type problems. Nenadov, Sudakov and Wagner [62] proposed the study of 'deficiency' problems for global spanning properties. The join $G * H$ of graph $G$ and $H$ is the graph obtained from taking vertex-disjoint copies of $G$ and $H$ and adding every edge between the copies. Now suppose that $G * K_{t}$ does not satisfy a particular graph property (such as being Hamiltonian). How many edges can $G$ have in terms of its order and $t$ ? Nenadov, Sudakov and Wagner [62] gave a complete answer whenever the property in question is Hamiltonicity, and gave a partial answer for the property of containing a triangle factor. Recently, Freschi, Hyde and Treglown [26] resolved the problem completely for the property of containing a $k$-clique factor, for any fixed $k \geqslant 3$.

Theorem 2.8 (Clique factors under deficiency conditions). Let $n, t, k \in \mathbb{N}$ such that $n \geqslant 2$, $t \geqslant 0$ and $k \geqslant 3$ such that $t<(k-1) n$ and $k$ divides $n+t$. Let $r=\lceil(t+1) /(k-1)\rceil$ and $q$ be the integer remainder when $t$ is divided by $k-1$. Let $G$ be a graph on $n$ vertices such that $G * K_{t}$ does not have a $K_{k}$-factor. Then $e(G) \leqslant g(n, t, k)$ where

$$
g(n, t, k)=\max \left\{\left(\begin{array}{l}
n \\
2
\end{array}\right)-\left(\begin{array}{c}
\frac{n+t}{k}+1 \\
2
\end{array}\right),\left(\begin{array}{l}
n \\
2
\end{array}\right)-\left(\begin{array}{l}
r \\
2
\end{array}\right)-r(n-r-(k-2-q))\right\} .
$$

These bounds are best possible when $k$ divides $n+t$, as shown by examples. Freschi, Hyde and Treglown [26] also showed an asymptotically optimal deficiency version of the Bandwidth Theorem for bipartite graphs, and asked for generalisations. The next results extends this to $k$-colourable graphs, for any $k \geqslant 3$.

Theorem 2.9 (Bandwidth Theorem under deficiency conditions). For $k, \Delta \in \mathbb{N}$ with $k \geqslant 2$ and $\mu>0$ there are $\beta, z>0$ and $n_{0} \in \mathbb{N}$ with the following property. Given $t \geqslant 0$, let $G$ be $a$ graph on $n \geqslant n_{0}$ vertices with $e(G) \geqslant g(n, k, t)+\mu n^{2}$, as defined in eq. (2.1). Then $G * K_{t}$ is $(z, \beta, \Delta, k)$-Hamiltonian.

2.5. Multipartite graphs. It is well known that Dirac's theorem also holds in bipartite graphs, where each part has size $n$ and each vertex has degree at least $n / 2$ [61]. To formulate extensions of this result, we introduce some further notation. Consider a balanced family $\mathcal{U}$ of $r$ disjoint sets each of size $n$. Let $G$ be a $\mathcal{U}$-partite graph, meaning that $G$ has no edges with both endpoints in the same part. The U-partite minimum degree of $G$, written $\delta(G ; \mathcal{U})$, is the least minimum degree of the bipartite subgraphs of $G$ induced by pairs of parts of $\mathcal{U}$. When $\mathcal{U}$ is clear from the context, we simply write $\delta_{r}(G)$.

Multipartite extensions of Dirac's theorem were first investigated in terms of clique factors. Fischer [25] conjectured a multipartite version of the Hajnal-Szemerédi theorem in balanced $r$-partite graphs. According to this, $\delta_{r}(G) \geqslant(k-1) n / k$ should be enough to find a $k$ clique factor in an $r$-partite graph $G$, of course assuming that the necessary condition that $k$ divides $r n$ holds. Examples by Catlin [12], later generalised by Keevash and Mycroft [39], 
show that if $r n / k$ is odd and $k$ divides $n$, then at least $\delta(G) \geqslant(k-1) n / k+1$ is necessary. The modified Fischer conjecture [47] posits that, nevertheless, those examples are the only cases where Fischer's original conjecture does not hold. This was confirmed for large $n$ and $k=r=3$ by Magyar and Martin [57], for $k=r=4$ by Martin and Szemerédi [58]. An approximate version was shown by Lo and Markström [55], and independently by Keevash and Mycroft [38]. Finally, Keevash and Mycroft [39] proved the full conjecture for all large $n$.

Theorem 2.10 (Multipartite Hajnal-Szemerédi Theorem). For $r, k \in \mathbb{N}$ with $r \geqslant k \geqslant 2$ and $\mu>0$, there exist $n_{0} \in \mathbb{N}$ such that for all $n \geqslant n_{0}$ where $k$ divides $r n$, the following holds. Let $G$ be a balanced $r$-partite graph on rn vertices with $\delta_{r}(G)>\frac{k-1}{k} n$. Then $G$ contains a $k$-clique factor.

Our next result extends this to powers of cycles and suitable $k$-colourable graphs. We add an extra piece of terminology to describe our results. A graph $H$ on $n k$ vertices admits an equitable $k$-colouring if it admits a $k$-colouring where each colour is used exactly $n$ times. A graph $G$ on $n k$ vertices is partite $(\beta, \Delta, k)$-Hamiltonian if $G$ contains every graph $H$ on $n k$ vertices with $\Delta(H) \leqslant \Delta$, bandwidth at most $\beta k n$ and an equitable $k$-colouring.

Theorem 2.11 (Multipartite Bandwidth Theorem). For $r, k, \Delta \in \mathbb{N}$ and $\mu>0$, there exist $\beta, z>0$ and $n_{0} \in \mathbb{N}$ such that the following holds. Let $G$ be a balanced $r$-partite graph on $r n$ vertices with $\delta_{r}(G) \geqslant\left(\frac{k-1}{k}+\mu\right) n$. It follows that

(i) if $r=k$, then $G$ is partite $(\beta, \Delta, k)$-Hamiltonian, and

(ii) if $r>k$, then $G$ is $(z, \beta, \Delta, k)$-Hamiltonian.

Note that $(k-1)$ th powers of tight cycles on $k n$ vertices admit equitable $k$-colourings, thus in particular Theorem 2.11 shows the existence of $(k-1)$ th powers of Hamilton cycles in $G$, in all cases. In the particular case of $(k-1)$ th powers of cycles, Theorem 2.11 was shown to be true very recently by DeBiasio, Martin and Molla [19], whose setting also allowed for suitably imbalanced partite graphs.

2.6. Robust expansion. Kühn, Osthus and Treglown [52] introduced the concept of robust expanders to study Hamilton cycles in dense graphs and digraphs. ${ }^{2}$ Given $0<\nu \leqslant \tau<1$, a graph $G$ on $n$ vertices and $S \subseteq V(G)$, the $\nu$-robust neighbourhood $\mathrm{RN}_{\nu, G}(S)$ of $S$ is the set of vertices $v \in V(G)$ with $\operatorname{deg}_{G}(v, S) \geqslant \nu n$. We say $G$ is a robust $(\nu, \tau)$-expander if for every $S \subseteq V(G)$ with $\tau n \leqslant|S| \leqslant(1-\tau) n$, we have $\left|\operatorname{RN}_{\nu, G}(S)\right| \geqslant|S|+\nu n$.

Robust expanders of sufficiently large minimum degree contain Hamilton cycles [52]. Knox and Treglown [41] extended this to the bandwidth setting.

Theorem 2.12 (Hamiltonicity under robust expansion). For all $\eta, \Delta>0$, there are $\tau, \nu, \beta>$ 0 with $\nu \leqslant \tau$ and $n_{0} \in \mathbb{N}$ such that the following holds. Let $G$ be a graph on $n \geqslant n_{0}$ vertices with $\delta(G) \geqslant \eta n$ which is a robust $(\nu, \tau)$-expander. Then $G$ is $(\beta, \Delta, 2)$-Hamiltonian.

We recover Theorem 2.12 by reducing it to our notion of Hamilton frameworks (Proposition 5.30). In fact, it can be observed that the notions of robust expansion (plus minimum degree) and robust Hamilton frameworks are essentially equivalent (Proposition 5.31).

In the next section, we introduce Hamilton frameworks which generalise the combination of robust expanders with linear minimum degree. Our main result then extends Theorem 2.12 to $(\beta, \Delta, k)$-Hamiltonicity for all $k \geqslant 2$.

\section{HAMilton FRAMEWORKS AND MAIN RESUlts}

In the following, we introduce and motivate the definition of our central notion, robust Hamilton frameworks, and then state our main results (Theorems 3.4 and 3.5).

\footnotetext{
${ }^{2}$ Similar concepts have been studied by Hefetz, Krivelevich and Szabó [33] and Brandt, Broersma, Diestel and Kriesell [10].
} 
3.1. Hamilton frameworks. To motivate the following definitions, consider a Hamiltonian graph $G$ that is (for sake of simplicity) non-bipartite. Then $G$ contains no isolated vertices, is connected, has a perfect fractional matching (as defined below) and contains an odd cycle. We call a graph that satisfies these properties a Hamilton framework. This property is not equivalent to Hamiltonicity as witnessed by two vertex-disjoint odd cycles connected by a single edge. Observe however that the framework properties of this construction are somewhat fragile. Deleting few vertices or few edges (at every vertex) may easily remove the properties of connectivity, having a perfect fractional perfect matching or an odd cycle.

To exclude examples like this, we could restrict our attention to Hamilton frameworks that are robust against such operations and ask again whether this already guarantees the existence of a Hamilton cycle. As it turns out, this the case. In fact, the same conditions even allow us to embed any bipartite graph $H$ of sublinear bandwidth and maximum degree into robust Hamilton frameworks $G$. If in addition $G$ robustly contains triangles, this can be extended to graphs $H$ with suitable zero-free 3-colourings. (Note that this condition is necessary, since such a $H$ might contain triangles.) This discussion corresponds to the case $k=2$. In the following we introduce the terminology to extend these ideas to powers of cycles and sublinear bandwidth graphs $H$ of higher chromatic number.

The general structural properties of Hamilton frameworks are formulated in terms of hypergraphs. In a $k$-uniform hypergraph (or $k$-graph for short) $\mathcal{H}$ every edge has exactly $k$ vertices. A tight (Hamilton) cycle $C \subseteq \mathcal{H}$ is a (spanning) subgraph whose vertices can be cyclically ordered such that its edges consist precisely of all sets of $k$ consecutive vertices in this ordering. For an (ordinary) graph $G$, we denote by $K_{k}(G)$ the $k$-clique (hyper)graph of $G$ which has vertex set $V(G)$ and a $k$-edge $X$ whenever $X$ induces a $k$-clique in $G$. We remark that $G$ contains the $(k-1)$ th power of a Hamilton cycle if and only if $K_{k}(G)$ contains a tight Hamilton cycle.

A (closed) tight walk in a $k$-graph $\mathcal{H}$ is a (cyclically) ordered sequence of vertices such that every set of $k$ consecutive vertices forms an edge of $\mathcal{H}$. Note that vertices and edges are allowed to be visited more than once in a closed tight walk. Moreover, a closed tight walk is a homomorphism of a tight cycle. The length of a tight walk is its number of vertices. We say that $\mathcal{H}$ is tightly connected if there is a closed tight walk that contains all its edges. Finally, a tight component of $\mathcal{H}$ is an edge-maximal tightly connected subgraph in $\mathcal{H}$.

A matching $M$ in a $k$-graph $G$ is a subgraph of vertex-disjoint edges. We use the following linear programming relaxation of matchings. A fractional matching is an edge weighting $\mathbf{w}: E(G) \rightarrow[0,1]$ such that $\sum_{e \ni v} \mathbf{w}(e) \leqslant 1$ for every vertex $v \in V(H)$. The size of $\mathbf{w}$ is the sum of its weights $\sum_{e \in E(G)} \mathbf{w}(e)$. A fractional matching is perfect if it attains the maximum possible size $v(G) / k$.

Given these preliminaries, we can now define general Hamilton frameworks.

Definition 3.1 (Hamilton framework). For $k \geqslant 2$, let $G$ be a graph and $\mathcal{H} \subseteq K_{k}(G)$ be a vertex-spanning subgraph. We say that $(G, \mathcal{H})$ is a $k$-uniform Hamilton framework if

(i) $\mathcal{H}$ is contained in a tight component of $K_{k}(G)$ and

(ii) $\mathcal{H}$ has a perfect fractional matching.

Moreover, the Hamilton framework $(G, \mathcal{H})$ is

(iii) aperiodic if $\mathcal{H}$ contains a closed tight walk of length coprime to $k$, and

(iv) zero-free if $\mathcal{H}$ contains a $(k+1)$-clique.

For convenience, we use the convention of writing '(aperiodic/zero-free) Hamilton framework' in the assumptions and outcomes of our results to mean that the Hamilton framework in question is aperiodic, zero-free or neither of the two.

3.2. Multipartite graphs. Our results concern graphs $G$, which may or may not be $k$ partite. To keep the presentation compact, we generally assume that $G$ is $\mathcal{U}$-partite with respect to some partition $\mathcal{U}$ consisting of $r$ parts where either $r=1$ or $r=k$, with the convention that $\mathcal{U}$-partiteness is vacuous when $r=1$. 
More precisely, consider a hypergraph $\mathcal{H}$ with an $(n, r)$-sized vertex partition $\mathcal{U}$, meaning that $\mathcal{U}$ has $r$ parts of size $n$ each. An edge $e \in E(\mathcal{H})$ is $\mathcal{U}$-partite if $e$ has at most one vertex in each part of $\mathcal{U}$, or if $r=1$. Similarly, $G$ is $\mathcal{U}$-partite if all edges of $G$ are $\mathcal{U}$-partite. We sometimes say that a Hamilton framework $(G, \mathcal{H})$ is $\mathcal{U}$-partite meaning that $G$ is $\mathcal{U}$-partite, note that in this case $\mathcal{H}$ must be $\mathcal{U}$-partite as well.

3.3. Robustness. As discussed before, we require our frameworks to be robust under small modifications of the original graph. We formalise this in terms of approximations.

Definition 3.2 (Approximation). Let $\varepsilon, d>0$ and $G$ be a $\mathcal{U}$-partite graph. A subgraph $G^{\prime} \subseteq G$ is a $\mathcal{U}$-partite $(\varepsilon, d)$-approximation of $G$ if for all $U, U^{\prime} \in \mathcal{U}$ and $v \in V\left(G^{\prime}\right)$, we have

(i) $\operatorname{deg}_{G^{\prime}}(v ; U) \geqslant \operatorname{deg}_{G}(v ; U)-d|U|$,

(ii) $\left|V\left(G^{\prime}\right) \cap U\right| \geqslant(1-\varepsilon)|U|$, and

(iii) $\left|V\left(G^{\prime}\right) \cap U\right|=\left|V\left(G^{\prime}\right) \cap U^{\prime}\right|$.

We also require that every vertex is appropriately connected to the hypergraph structure of frameworks. Consider an edge $e$ and a vertex $v \notin e$ in a $k$-graph. We say that $v$ is linked to $e$ if there is an edge $f$ with $v \in f$ and $|e \cap f|=k-1$. For two $k$-graphs $G_{1}=\left(V_{1}, E_{1}\right)$ and $G_{2}=\left(V_{2}, E_{2}\right)$, we write $G_{1} \cap G_{2}=\left(V_{1} \cap V_{2}, E_{1} \cap E_{2}\right)$.

With these definitions at hand, we can describe robust frameworks $(G, \mathcal{H})$ using subgraphs $\mathcal{H} \subseteq K_{k}(G)$ which preserve their properties after passing to any approximation; and which in addition also have many linked edges at every vertex.

Definition 3.3 (Robustness). Let $G$ be a $\mathcal{U}$-partite graph, with an $(n, r)$-sized partition $\mathcal{U}$, and let $\mathcal{H} \subseteq K_{k}(G)$. We say that $(G, \mathcal{H})$ is a $\mu$-proto-robust $\mathcal{U}$-partite (aperiodic/zero-free) Hamilton framework if

(R1) $\left(G^{\prime}, \mathcal{H}^{\prime}\right)$ is an (aperiodic/zero-free) Hamilton framework for each $\mathcal{U}$-partite $(\mu, \mu)$ approximation $G^{\prime}$ of $G$ where $\mathcal{H}^{\prime}=\mathcal{H} \cap K_{k}\left(G^{\prime}\right)$.

Moreover, $(G, \mathcal{H})$ is called $\mu$-robust if addition to this

(R2) each $v \in V(G)$ has at least $\mu n^{k}$ linked edges in $\mathcal{H}$.

As a convention, we omit the decorator ' $U$-partite' when $r=1$ in this definition.

3.4. Robust Hamilton frameworks are Hamiltonian. Now we formulate our main results from which the outcomes in Section 2 can be derived. The first theorem states that robust aperiodic Hamilton frameworks contain powers of Hamilton cycles.

Theorem 3.4 (Frameworks - cycles). For $k \in \mathbb{N}$ and $\mu>0$ there exists $n_{0} \in \mathbb{N}$ with the following property. Let $(G, \mathcal{H})$ be a k-uniform $\mu$-robust aperiodic Hamilton framework on $n \geqslant n_{0}$ vertices. Then $G$ contains the $(k-1)$ th power of a Hamilton cycle.

The second theorem shows that graphs which admit $k$-uniform robust frameworks contain different kinds of bounded-degree sublinear-bandwidth spanning graphs, depending on whether the framework is partite, aperiodic or zero-free.

Theorem 3.5 (Frameworks - bandwidth). For $k, \Delta \in \mathbb{N}, \mu>0$, and $r=1$ or $r=k$ there are $\beta, z$ and $n_{0} \in \mathbb{N}$ with the following property. Let $(G, \mathcal{H})$ be a $\mu$-robust $\mathcal{U}$-partite $k$-uniform Hamilton framework where $\mathcal{U}$ is an $(n, r)$-sized partition of $G$ with $n \geqslant n_{0}$.

(i) If $r=k$, then $G$ is $\mathcal{U}_{\text {-partite }}(\beta, \Delta, k)$-Hamiltonian.

(ii) If $(G, \mathcal{H})$ is $\mu$-robust aperiodic, then $G$ is $(\beta, \Delta, k)$-Hamiltonian.

(iii) If $(G, \mathcal{H})$ is $\mu$-robust zero-free, then $G$ is $(z, \beta, \Delta, k)$-Hamiltonian.

3.5. Remarks on frameworks. We reflect briefly about our definition of Hamilton frameworks, robustness and our main results.

Firstly, unlike for Hamilton cycles, searching for Hamilton frameworks is computationally tractable (see Appendix B.1). This indicates, on a theoretical level, that Hamilton frameworks are 'simpler' structures. It is moreover conceivable, that the proofs of Theorem 3.4 
and 3.5 lead to efficient algorithms for constructing spanning structures given a robust framework as an input (see Section 11).

Secondly, it is not hard to see that in a Hamilton framework, every vertex has at least one linked edge. One could suspect that in robust framework every vertex has many linked edges. However, we were only able prove this when $\mathcal{H}=K_{k}(G)$.

Proposition 3.6 (Linked edges). For $k \in \mathbb{N}$ and $\mu>0$ there are $0<\mu^{\prime} \leqslant \mu$ and $n_{0} \in \mathbb{N}$ such that every $\mathcal{U}$-partite $\mu$-proto-robust $k$-uniform (aperiodic/zero-free) Hamilton framework $\left(G, K_{k}(G)\right)$ on $n \geqslant n_{0}$ vertices is $\mu^{\prime}$-robust.

Proposition 3.6 is a simple consequence of the Graph Removal Lemma. Its proof can be found in Appendix B.2.

Thirdly, there are interesting graphs $G$ in which $K_{k}(G)$ contains a tight Hamilton cycle but is not tightly connected. It is furthermore very convenient for our proofs to allow that $\mathcal{H} \subseteq K_{k}(G)$ must not be tightly connected itself (see Section 5.4).

Next, we note that 'being a robust Hamilton framework' is stronger than 'robustly having a Hamilton framework', by which we mean having some framework after the edge and vertex deletion. This stricter assumption is necessary, as there are graphs with the latter property which are not Hamiltonian (see Appendix A.3).

Finally, it is possible to relax Hamilton frameworks to almost perfect fractional matchings. More precisely, Theorems 3.4 and 3.5 remain valid if for $\varrho \geqslant 0$ small enough with respect to $\beta, z, k, \Delta, \mu$, we require that $\mathcal{H}$ has a fractional matching of size at least $(1-\varrho) v(G) / k$. If $\varrho>0$, we need as a further assumption that every vertex of $G$ lies in an edge of $\mathcal{H}$, which is implicit when $\varrho=0$.

3.6. Methodology. Let us explain how we prove our main results. The approach rests on a combination of graph regularity and the Blow-Up Lemma. To overcome obstacles that arise in our general setting, we require a series of new ideas, which we describe in the following. For simplicity, we focus in the aperiodic case. The proof of Theorem 3.4 is based on two lemmas. Given an aperiodic Hamilton framework $(G, \mathcal{H})$, we first apply Lemma 8.2 (Lemma for $G$ - cycles) to find a regular partition $\mathcal{V}$ of bounded size, together with a reduced graph $R$ whose vertices are the clusters of $\mathcal{V}$. It turns out we can find $\mathcal{J} \subseteq K_{k}(R)$ so that $(R, \mathcal{J})$ is an aperiodic Hamilton framework. This will mean that $(R, \mathcal{J})$ approximately captures the structure of $(G, \mathcal{H})$. Lemma 8.2 also provides a spanning subgraph $R^{\prime} \subseteq R$ of bounded maximum degree, such that $G$ has stronger regularity properties with respect to $R^{\prime}$ (it consists of super-regular pairs). We then use Lemma 8.3 (Lemma for $C$ ) to allocate a power of a Hamilton cycle $C$ to a $\mathcal{V}$-blow-up $R^{*}$ of $R$. Finally, we apply the Blow-Up Lemma to turn this allocation into an embedding of $C$ in $G$.

The proof of Lemma 8.2 (Lemma for $G$ - cycles) begins similar as in the setting of minimum degree conditions. However, incorporating certain exceptional vertices (into the structure described by $R^{\prime}$ ) turns out to be much more complicated and requires additional absorption arguments. In the proof of Lemma 8.3 (Lemma for $C$ ), a new obstacle appears. If the partition $\mathcal{V}$ is balanced after a few intermediate preparations, we can effectively turn a perfect fractional matching of $\mathcal{J}$ into the desired allocation. Unfortunately (and unlike in case of minimum degree conditions), Lemma 8.2 returns a partition $\mathcal{V}$ that is too unbalanced for this argument. We deal with this by showing that $(R, \mathcal{J})$ inherits a stronger matching property (called 'cluster-matchability'), which can handle the varying cluster sizes of $\mathcal{V}$. This allows us to find an allocation that covers most of the vertices. The last few vertices are then allocated using the aperiodicity of the framework.

The proof of Theorem 3.5 has broadly the same structure as the one of Theorem 3.4. As before, the proof is based on two lemmas: Lemma 9.1 (Lemma for $G$ - bandwidth) and Lemma 9.2 (Lemma for $H$ ). The difference between Lemma 8.2 and Lemma 9.1 is that the latter returns a subgraph $R^{\prime} \subseteq R$, which is not only a bounded-degree graph but, more specifically, is a power of a cycle; this is obtained by applying Theorem 3.4 to $R$. Unfortunately, this complicates matters when including exceptional vertices into $R^{\prime}$. We 
therefore have to ensure that $R^{\prime}$ runs through a set of predefined edges of $\mathcal{J}$ (the hypergraph part of the reduced framework), which effectively means that we have to prove a stronger result on Hamilton cycles (Theorem 8.1).

In the second part of the proof of Theorem 3.5, we use Lemma 9.2 to allocate a given graph $H$ to the $\mathcal{V}$-blow-up of $R^{*}$ of the reduced graph $R$. This allocation proceeds in several steps. We first allocate $H$ greedily to a blow-up of a power of a path $P^{*}$, where the sizes of the blown-up clusters of $P^{*}$ may differ by a lot. Next, we allocate $P^{*}$ to the blow-up of a power of a cycle $C^{*}$, whose blown-up clusters are almost completely balanced. This is done by allocating the clusters of $P^{*}$ by means of a random walk on the clusters of $C^{*}$, which is ultimately possible thanks to the aperiodicity of the Hamilton framework $(G, \mathcal{H})$. If $\mathcal{V}$ was a balanced partition, we could simply set the blow-up size of $C$ to the cluster size of $\mathcal{V}$ and take $R^{\prime}=C$. However, in general, we can only assume that $\mathcal{V}$ is almost balanced. We deal with this by taking the cluster size of $C$ to be much smaller than the cluster size of $\mathcal{V}$ and then allocating $C^{*}$ into $R^{*}$ with another application of Lemma 8.3 (Lemma for $C$ ). Together, this results in an allocation that is very close to the cluster size of $\mathcal{V}$. As a final step (before applying the Blow-Up Lemma), we modify the partition $\mathcal{V}$ to precisely fit this allocation. This can be done with a simple (but novel) shifting argument, which again relies on the aperiodicity of the framework.

Finally, in the situations where the Hamilton framework is partite or zero-free, the proof takes a very similar shape. For the case of zero-freeness, much stays the same as zero-freeness implies aperiodicity. Only in Lemma 9.2 we have to a bit more careful, when embedding the vertices of colour 0. More details on this can be found in Section 9. The partite version of Theorem 3.5 is actually easier to prove. This is because most of the arguments involving aperiodicity can be replaced by simple observations on the partite assumptions on $(G, \mathcal{H})$ and $H$.

3.7. Organisation of the paper. The rest of the paper is organised as follows. The next section contains some basic definitions and notation. In Section 5, we derive the results of Section 2 from Theorems 3.4 and 3.5. The second part of the paper is dedicated to the proofs of Theorems 3.4 and 3.5. In Sections 6 and 7, we introduce tools and prove a few intermediate results. Sections 8 and 9 contain the proof of Theorems 3.4 and 3.5, respectively. The latter depends on Lemma 9.2 (Lemma for $H$ ), which is proved in Section 10. We conclude the paper with a few open problems and reflections in Section 11. Some further constructions as well as proofs of standard and borrowed results can be found in the appendix.

\section{Notation}

In this section, we introduce some general notation that will be used throughout the paper. A hypergraph $\mathcal{H}$ consists of a set of vertices $V(\mathcal{H})$ and edges $E(\mathcal{H})$, where the edges are subsets of vertices. We say that a hypergraph is $k$-uniform (or $k$-graph for short) if all edges have size $k$. So, graphs are simply 2-graphs. A set of $X$ size $k$ is a $k$-set, or a $k$-edge if $X$ is an edge in a hypergraph.

For a hypergraph $\mathcal{H}$, a set $U \subseteq V(\mathcal{H})$, and $v \in V(\mathcal{H})$, the neighbours $N_{\mathcal{H}}(v ; U)$ of $v$ in $U$ is the collection of sets $X \subseteq U \backslash\{v\}$ such that $X \cup\{v\} \in E(\mathcal{H})$. The degree of $v$ in $U$ is $\operatorname{deg}_{\mathcal{H}}(v ; U)=\left|N_{\mathcal{H}}(v ; U)\right|$. If $U=V(\mathcal{H})$, we just write $N_{\mathcal{H}}(v)$ and $\operatorname{deg}_{\mathcal{H}}(v)$. Finally, if $\mathcal{H}$ is clear from the context, we omit its references in the index. We denote by $\mathcal{H}[U]$ the induced hypergraph of $\mathcal{H}$ on $U$. A homomorphism from a $k$-graph $\mathcal{H}_{1}$ to a $k$-graph $\mathcal{H}_{2}$ is a function $\phi: V\left(\mathcal{H}_{1}\right) \rightarrow V\left(\mathcal{H}_{2}\right)$, which maps edges of $\mathcal{H}_{1}$ to edges of $\mathcal{H}_{2}$. An embedding is an injective homomorphism. The maximum degree $\Delta(\mathcal{H})$ is defined as the maximum of $\operatorname{deg}_{\mathcal{H}}(v)$ over all vertices $v \in V(\mathcal{H})$. For $1 \leqslant i \leqslant k$, we define the $i$ th shadow of $\mathcal{H}$, denoted $\partial_{i} \mathcal{H}$, as the $i$-graph on $V(\mathcal{H})$ which contains all $i$-edges that are a subset of an edge of $\mathcal{H}$. A tight path in a $k$-graph is a tight walk which does not repeat vertices. For a $k$-graph $\mathcal{H}$ and $X_{1}, X_{2} \in V(\mathcal{H})^{k-1}$, a tight $\left(X_{1}, X_{2}\right)$-path in $\mathcal{H}$ is a tight path in $\mathcal{H}$ that starts with the vertices of $X_{1}$ and ends with the vertices of $X_{2}$. 
To express the constant hierarchies in the results and definitions, we use the following standard notation. We write $x \ll y$ to mean that for any $y \in(0,1]$ there exists an $x_{0} \in(0,1)$ such that for all $x \leqslant x_{0}$ the subsequent statements hold. Hierarchies with more constants are defined in a similar way and are to be read from the right to the left. Implicitly, we will assume constants appearing in a hierarchy are positive real numbers. Moreover, if $1 / x$ appears in a hierarchy we will further assume $x$ is a natural number. Finally, for a set $X$ and a tuple $\mathbf{v} \in \mathbb{R}^{X}$, we let $\|\mathbf{v}\|_{\infty}=\max _{x \in X}|\mathbf{v}(x)|$ and $\|\mathbf{v}\|_{1}=\sum_{x \in X}|\mathbf{v}(x)|$.

\section{Proof of the applications}

In this section, we show the applications detailed in Section 2.

5.1. Observations on tight connectivity. In this subsection we make two elementary but useful observations about tight connectivity. ${ }^{3}$ The following proposition implies that the tight components of a $k$-graph $\mathcal{H}$ form a partition of its edge set, and hence justifies the notation of tight components.

Proposition 5.1. Let $X$ and $Y$ be two edges in a k-graph which intersect in $k-1$ vertices. Then $X$ and $Y$ are in the same tight component.

Proof. Consider an arbitrary closed tight walk $W$, which visits the edge $X$. To prove the proposition, it suffices to show that there is a tight closed walk $W^{\prime}$ which covers the same edges as $W$ and in addition $Y$. Since $W$ contains $X$, there is a subwalk $\mathcal{X}=\left(x_{1}, \ldots, x_{k}\right)$ of $W$ such that $X=\left\{x_{1}, \ldots, x_{k}\right\}$. By assumption, we have $|X \cap Y|=k-1$, so $X \backslash Y=\left\{x_{j}\right\}$ for some $1 \leqslant j \leqslant k$ and there exists a vertex $y$ such that $Y \backslash X=\{y\}$. It follows that

$$
\mathcal{Y}:=\left(x_{1}, \ldots, x_{k}, x_{1}, \ldots, x_{j-1}, y, x_{j+1}, \ldots, x_{k}, x_{1}, \ldots, x_{k}\right)
$$

is a tight walk which visits $X$ and $Y$. Hence we can replace $\mathcal{X}$ with $\mathcal{Y}$ in $W$ to obtain a tight closed walk $W^{\prime}$ which covers all edges covered by $W$ and also $Y$.

Given a $k$-graph $\mathcal{H}$ and a vertex $v$, we define the link graph $L_{\mathcal{H}}(v)$ to be the $(k-1)$-graph with vertex set $V(\mathcal{H})$ and edge set $N_{\mathcal{H}}(v)=\{X:\{v\} \cup X \in E(\mathcal{H})\}$.

Proposition 5.2. Let $\mathcal{H}$ be a k-graph and $v \in V(\mathcal{H})$. Suppose that $L_{\mathcal{H}}(v)$ is tightly connected. Then the edges of $\mathcal{H}$ containing $v$ are in the same tight component.

Proof. By assumption, there is a tight walk $W$ in $L_{\mathcal{H}}(v)$ that visits all of its edges. Let $\left(u_{1}, \ldots, u_{k}\right) \subseteq W$ be a tight subwalk. By Proposition 5.1, the edges $\left\{u_{1}, \ldots, u_{k-1}, v\right\}$ and $\left\{u_{2}, \ldots, u_{k}, v\right\}$ are in the same tight component of $\mathcal{H}$. We conclude by iterating this observation along all the vertices of $W$.

5.2. Pósa-type conditions. The goal of this subsection is to prove Theorem 2.6 (Bandwidth theorem under Pósa-type conditions). All we require in addition to the results of Sections 2 and 3 is the following lemma.

Lemma 5.3. Let $G$ be a graph with vertices $v_{1}, \ldots, v_{n}$. Suppose that $\operatorname{deg}\left(v_{i}\right)>(k-2) n / k+$ $\min \{i, n / k\}$ for every $i \leqslant n$. Then $K_{k}(G)$ is tightly connected, spans all vertices of $G$, and contains a $(k+1)$-clique.

Given Lemma 5.3, we can easily combine Theorem 2.5 (Clique factors under Pósa-type conditions) and Theorem 3.5 to derive the main result of this section.

Proof of Theorem 2.6. We can assume $\mu \leqslant 1 / 2$, as otherwise the statement is vacuous. Let $\mu^{\prime}, n_{1}$ be given by Proposition 3.6 under input $k, \mu / 2$. Let $n_{2}$ be given by Theorem 2.5 under input $k, \mu / 4$. Let $\beta, z>0$, and $n_{3} \in \mathbb{N}$ be obtained from Theorem 3.5 applied with $k, \Delta, \mu^{\prime}$, and let $n_{0}=\max \left\{n_{1},(1+\mu)\left(n_{2}+k^{2}\right), n_{3}, 8 \mu^{-1} k^{2}\right\}$.

Let $n \geqslant n_{0}$ and consider a graph $G$ with degree sequence $d_{1}, \ldots, d_{n}$ such that $d_{i} \geqslant$ $\frac{k-2}{k} n+i+\mu n$ for every $i \leqslant n / k$. Let $\mathcal{H}=K_{k}(G)$. By Theorem 3.5 it is enough to show that

\footnotetext{
${ }^{3}$ For a more systematic investigation of tight connectivity, see our work on tight Hamilton cycles under minimum degree conditions [53].
} 
$(G, \mathcal{H})$ is a $\mu^{\prime}$-robust zero-free Hamilton framework. By Proposition 3.6 and the choice of $\mu^{\prime}, n_{0}$, it is enough to show that $(G, \mathcal{H})$ is a $(\mu / 2)$-proto-robust zero-free Hamilton framework.

To see this, let $G^{\prime} \subseteq G$ be a ( $\left.\mu / 2\right)$-approximation of $G$ and let $\mathcal{H}^{\prime}=\mathcal{H} \cap K_{k}\left(G^{\prime}\right)$. Let $n^{\prime}$ be the number of vertices of $G^{\prime}$, and note that $n^{\prime} \geqslant(1-\mu / 2) n$. Moreover $G^{\prime}$ satisfies the same Pósa-type degree conditions as $G$, now with $\mu / 2$ instead of $\mu$. Consequently, $\mathcal{H}^{\prime}$ is tightly connected and contains a $(k+1)$-clique by Lemma 5.3. In fact, iterating this observation we can obtain $q$ such cliques where $q$ is the integer remainder when dividing $n^{\prime}$ by $k$. Removing these cliques leaves a graph $G^{\prime \prime}$ on at least $n^{\prime}-k^{2} \geqslant n_{2}$ vertices. Since $\mu \leqslant 1 / 2$ and $n \geqslant 8 \mu^{-1} k^{2}$, we have $\mu n^{\prime} / 4 \geqslant k^{2}$. This implies $G^{\prime \prime}$ retains its Pósa-type conditions, but now with $\mu / 4$ instead of $\mu$. By Theorem 2.5, $G^{\prime \prime}$ has a $k$-clique factor $M$. Thus, we can define a perfect fractional matching in $G^{\prime}$ by assigning weight 1 to the cliques of $M$ and weight $1 / k$ to the edges of the $(k+1)$-cliques. This shows that $\left(G^{\prime}, \mathcal{H}^{\prime}\right)$ is a zero-free Hamilton framework, and finishes the proof.

The rest of this subsection is dedicated to the proof of Lemma 5.3. Note that the assumptions of the lemma imply that $n \geqslant k+1$. Given the statement of Lemma 5.3, we say a vertex $v$ of $G$ is big if $\operatorname{deg}_{G}(v) \geqslant(k-1) n / k$. Note that if $k \geqslant 3$, then there are more than $2 n / k$ big vertices in $G$. The following observation clears the way for an induction on $k$.

Proposition 5.4. Given the assumptions of Lemma 5.3 and $k \geqslant 3$, let $v$ be a big vertex, $G^{\prime}=G[N(v)]$ and $n^{\prime}=\left|V\left(G^{\prime}\right)\right|$. Then $\operatorname{deg}_{G^{\prime}}\left(v_{j}\right)>\frac{k-3}{k-1} n^{\prime}+\min \left\{j, \frac{n^{\prime}}{k-1}\right\}$ for each $v_{j} \in V\left(G^{\prime}\right)$.

Proof. Let $v_{j}$ be a vertex in $V\left(G^{\prime}\right)$. Using that $\operatorname{deg}_{G}(v)=n^{\prime}>(k-1) n / k$, we obtain

$$
\begin{aligned}
\operatorname{deg}_{G^{\prime}}\left(v_{j}\right) & \geqslant\left|N_{G}\left(v_{j}\right) \backslash\left(V(G) \backslash N_{G}(v)\right)\right| \geqslant \operatorname{deg}_{G}\left(v_{j}\right)-\left(n-\operatorname{deg}_{G}(v)\right) \\
& =\frac{k-2}{k} n+\min \left\{j, \frac{n}{k}\right\}-\left(n-n^{\prime}\right) \geqslant n^{\prime}-\left(\frac{2}{k}-\min \left\{\frac{j}{n}, \frac{1}{k}\right\}\right) n \\
& >n^{\prime}-\left(\frac{2}{k}-\min \left\{\frac{j}{n}, \frac{1}{k}\right\}\right) \frac{k}{k-1} n^{\prime} \geqslant \frac{k-3}{k-1} n^{\prime}+\min \left\{\frac{k}{k-1} \frac{j}{n}, \frac{1}{k-1}\right\} n^{\prime} \\
& \geqslant \frac{k-3}{k-1} n^{\prime}+\min \left\{j, \frac{n^{\prime}}{k-1}\right\} .
\end{aligned}
$$

Proof of Lemma 5.3. We go by induction on $k$. The case of $k=2$ is simple. By Pósa's theorem, $G$ contains a Hamilton cycle, thus $K_{2}(G)=G$ is spanning and tightly connected. A vertex $v$ of maximum degree in $G$ has $\operatorname{deg}_{G}(v)>n / 2$, consequently any Hamilton cycle in $G$ must have an edge in $N(v)$. This implies $G$ contains a triangle, as needed.

Now let us assume that $k \geqslant 3$ and the statement holds for all $k^{\prime}<k$. Consider a graph $G$ with vertices $v_{1}, \ldots, v_{n}$ as in the statement and abbreviate $\mathcal{H}=K_{k}(G)$. Let $v$ be a big vertex. By Proposition 5.4 and induction, the link graph $L_{\mathcal{H}}(v)$ contains a $k$-clique. Hence $G$ contains a $k+1$ clique.

Showing that $\mathcal{H}$ is tightly connected is a bit more involved. We proceed in three steps. First, we prove that the edges in $\mathcal{H}$ that contain a big vertex $v$ are in a common tight component $\mathcal{H}_{v}$. Next, we show that there is a tight component $\mathcal{H}^{\prime}$ such that $\mathcal{H}^{\prime}=\mathcal{H}_{v}$ for all big vertices $v$. Finally, we prove that $\mathcal{H}^{\prime}$ covers in fact all edges of $\mathcal{H}$. Now come the details.

Observation 5.5. If $v$ is a big vertex, then $L_{\mathcal{H}}(v)$ is tightly connected and spans $N_{G}(v)$.

Proof of the claim. Indeed, note that all edges of $L_{\mathcal{H}}(v)$ are contained in $N_{G}(v)$. So we can restrict our analysis to $G^{\prime}=G\left[N_{G}(v)\right]$. Since $k \geqslant 3$, Proposition 5.4 tells us that $G^{\prime}$ satisfies the induction hypothesis with $k^{\prime}=k-1 \geqslant 2$, so we get that $K_{k-1}\left(G^{\prime}\right)=L_{\mathcal{H}}(v)$ is tightly connected and spanning in $V\left(G^{\prime}\right)=N_{G}(v)$, as desired.

Observation 5.6. If $v$ is a big vertex and $u \in N_{G}(v)$, then $u$ and $v$ belong to a common $k$-clique of $G$.

Proof of the claim. By Observation 5.5, $L_{\mathcal{H}}(v)$ is tightly connected and spans all $N_{G}(v)$. Since $u \in N_{G}(v)$, in particular $u$ belongs to a $(k-1)$-edge of $L_{\mathcal{H}}(v)$, which is equivalent to say that $u$ lies in a $(k-1)$-clique inside $N_{G}(v)$, and thus in a $k$-clique together with $v$. 
Now it is easy to see that $\mathcal{H}$ is spanning.

Observation 5.7. Every vertex of $G$ is contained in a $k$-clique.

Proof of the claim. Let $v$ be a vertex of $G$. Using $\operatorname{deg}_{G}(v)>(k-2) n / k+1$ and the fact that there are more than $2 n / k$ big vertices in $G$, we deduce that there exists a big vertex $w$ such that $w \in N_{G}(v)$. It follows by Observation 5.6 that $v$ is contained in a $k$-clique.

Together with Proposition 5.2, Observation 5.5 implies that, given a big vertex $v$, all the $k$-edges containing $v$ belong to the same tight component of $\mathcal{H}$. We will denote that component by $\mathcal{H}_{v}$.

Observation 5.8. If $u, v$ are big vertices, then $\mathcal{H}_{u}=\mathcal{H}_{v}$.

Proof of the claim. Since $u$ and $v$ are big, their common neighbourhood contains at least $(k-2) n / k$ vertices. Since there are more than $2 n / k$ big vertices in $G$, there exists a big vertex $w$ adjacent to both $u$ and $v$. By Observation 5.6, there exists a $k$-clique $X$ containing both $u$ and $w$. Since $u$ and $w$ are big vertices, then $X$ belongs to $\mathcal{H}_{w}$ as well as $\mathcal{H}_{u}$, hence $\mathcal{H}_{u}=\mathcal{H}_{w}$. By symmetry, also $\mathcal{H}_{v}=\mathcal{H}_{w}$ and thus $\mathcal{H}_{u}=\mathcal{H}_{v}$.

By Observation 5.8, there exists a tight component $\mathcal{H}^{\prime}$ of $\mathcal{H}$ which contains $\mathcal{H}_{u}$ for every big vertex $u$. By Observation 5.7, we can finish the proof by showing that $\mathcal{H}^{\prime}$ actually contains all edges of $\mathcal{H}$.

For the sake of contradiction, suppose that this is not true. Consider an edge $X=$ $\left\{v_{i_{1}}, \ldots, v_{i_{k}}\right\}$ in $\mathcal{H}$, which is not in $\mathcal{H}^{\prime}$ and maximises $\sum_{j} i_{j}$. Suppose $i_{1}<\cdots<i_{k}$. If $X$ contains a big vertex $u$, then $X \in E\left(\mathcal{H}_{u}\right)=\mathcal{H}^{\prime}$, a contradiction. We therefore must have $i_{j}<n / k$ for all $1 \leqslant j \leqslant k$. Let $e(X)$ and $e(X, V \backslash X)$ be the number of edges inside $X$, and the number of edges with exactly one endpoint in $X$, respectively. On one hand, we have

$$
\begin{aligned}
e(X, V \backslash X) & =\sum \operatorname{deg}_{G}\left(v_{i_{j}}\right)-2 e(X) \geqslant k\left(\frac{k-2}{k}\right) n+\sum i_{j}-2\left(\begin{array}{l}
k \\
2
\end{array}\right) \\
& =(k-2) n+\sum i_{j}-k(k-1) .
\end{aligned}
$$

Define $I_{1}=\left\{1, \ldots, i_{1}-1\right\}, I_{2}=\left\{i_{1}, \ldots, i_{k}\right\}, I_{3}=\left\{i_{k}+1, \ldots, n\right\}$, and for all $1 \leqslant i \leqslant 3$ let $V_{i}=\left\{v_{j}: j \in I_{i}\right\} \backslash X$. Note that $\left|V_{1}\right|=i_{1}-1,\left|V_{2}\right|=\left|I_{2}\right|-|X|=i_{k}-i_{1}+1-k$ and $\left|V_{3}\right|=n-i_{k}$. In the following, we show that

(a) every vertex in $V_{1}$ has at most $k$ neighbours in $X$,

(b) every vertex in $V_{2}$ has at most $k-1$ neighbours in $X$, and

(c) every vertex in $V_{3}$ has at most $k-2$ neighbours in $X$.

Part (a) is trivial. For part (b), let us suppose otherwise and obtain a contradiction. Then there exists $\ell>i_{1}$ such that $v_{\ell}$ which has $k$ neighbours in $X$. Let $X^{\prime}=\left(X \backslash\left\{v_{i_{1}}\right\}\right) \cup\left\{v_{\ell}\right\}$. It follows that $X^{\prime}$ is an edge in $\mathcal{H}$ with a higher sum of indices as $X$. Moreover, $X^{\prime}$ is in the same tight component as $X$ by Proposition 5.1, a contradiction. Similarly, for part (c), suppose otherwise for sake of contradiction. Then there exists $\ell>i_{k}$ such that $v_{\ell}$ which has at least $k-1$ neighbours in $X$. Let $X^{\prime}$ be a $k$-clique obtained from $X$ by replacing a vertex not adjacent to $v_{\ell}$ with $v_{\ell}$. (If $v_{\ell}$ is adjacent to all vertices of $X$, just replace an arbitrary vertex.) As before, $X^{\prime}$ is an edge in $\mathcal{H}$ in the same component as $X$ and with a higher sum of indices as $X$, contradiction.

Putting observations (a) to (c) together, we get

$$
\begin{aligned}
e(X, V \backslash X) & \leqslant\left|V_{1}\right| k+\left|V_{2}\right|(k-1)+\left|V_{3}\right|(k-2) \\
& =\left(i_{1}-1\right) k+\left(i_{k}-i_{1}-k+1\right)(k-1)+\left(n-i_{k}\right)(k-2) \\
& =(k-2) n+i_{1}+i_{k}-k(k-1)-1 \\
& <(k-2) n+\sum i_{j}-k(k-1),
\end{aligned}
$$

which contradicts inequality (5.1) and finishes the proof. 
5.3. Ore-type conditions. In this section, we show Theorem 2.4 (Bandwidth theorem under Ore-type conditions). The general strategy follows what we have done in the last section. Recall that that a graph $G$ is $\delta$-Ore if $\operatorname{deg}(x)+\operatorname{deg}(y)>2 \delta v(G)$ for all $x y \notin E(G)$. In such a graph, we also say that a vertex $x$ is $\delta$-big if $\operatorname{deg}(x)>\delta n$, and otherwise is $\delta$-small. If the context is clear, we just say big and small instead. Note that the set of small vertices in a $\delta$-Ore graph forms a clique under this definition.

Unlike Dirac-type conditions, Ore-type conditions are not necessarily inherited by approximations, for instance when an edge between two small vertices is deleted. We will therefore need stronger structural insights before applying Theorem 3.5.

We show the following two lemmas.

Lemma 5.9. Let $1 / n \ll \nu \ll \mu, 1 / k$ and let $G$ be $a\left(\frac{k-1}{k}+\mu\right)$-Ore graph on $n$ vertices. Let $G^{\prime}$ be a $\nu$-approximation of $G$. Then $K_{k}\left(G^{\prime}\right)$ is tightly connected, spans all vertices, and big vertices are on a clique of size $k+1$.

Lemma 5.10. Let $1 / n \ll \nu \ll \mu, 1 / k$. Let $G$ be a $\left(\frac{k-1}{k}+\mu\right)$-Ore graph on $n$ vertices. Let $G^{\prime}$ be a $\nu$-approximation of $G$. Then $K_{k}\left(G^{\prime}\right)$ has a perfect fractional matching.

Given Lemmas 5.9 and 5.10, one can derive Theorem 2.4 from Theorem 3.5 in the same way as it was done for the proof of Theorem 2.6 (Bandwidth theorem under Pósa-type conditions). We omit the details.

The rest of the subsection is dedicated to the proof of Lemmas 5.9 and 5.10. We start by showing the following.

Lemma 5.11. Let $G$ be a $\frac{k-1}{k}$-Ore graph on $n \geqslant k+1$ vertices. Then $K_{k}(G)$ is tightly connected, spans all vertices, and all big vertices are on a clique of size $k+1$.

Note that, since $n \geqslant k+1$ in this lemma, the set of big vertices is never empty. The following observation clears the way for an induction on $k$.

Proposition 5.12. Let $G$ be a $\frac{k-1}{k}$-Ore graph on $n>k$ vertices with $k \geqslant 3$. Let $v$ be a big vertex, $G^{\prime}=G[N(v)]$ and $n^{\prime}=\left|V\left(G^{\prime}\right)\right|$. Then $G^{\prime}$ is $\frac{k-2}{k-1}$-Ore with $n^{\prime}>k-1$.

Proof. Since $n>k$ and $n^{\prime}=\operatorname{deg}_{G}(v)>(k-1) n / k$, we have $n^{\prime}>k-1$. Let $x, y$ be vertices in $V\left(G^{\prime}\right)$ with $x y \notin E\left(G^{\prime}\right)$. Using $n^{\prime}>(k-1) n / k$, we obtain

$$
\begin{aligned}
\operatorname{deg}_{G^{\prime}}(x)+\operatorname{deg}_{G^{\prime}}(y) & \geqslant \operatorname{deg}_{G}(x)+\operatorname{deg}_{G}(y)-2\left(n-\operatorname{deg}_{G}(v)\right) \\
& >2 \frac{k-1}{k} n-2 \frac{k}{k} n+2 \operatorname{deg}_{G}(v)=2 \operatorname{deg}_{G}(v)-2 \frac{1}{k} n \\
& \geqslant 2 n^{\prime}-2 \frac{1}{k-1} n^{\prime}=2 \frac{k-2}{k-1} n^{\prime} .
\end{aligned}
$$

Proof of Lemma 5.11. We go by induction on $k$. If $k=2$, Ore's theorem implies the existence of a Hamilton cycle, so $K_{2}(G)=G$ is tightly connected. Let $x$ be a big vertex in $G$ and note that $\operatorname{deg}(x)>n / 2$. Hence any Hamilton cycle of $G$ must have an edge in $N(x)$. It follows that $G$ has a triangle. This concludes the case $k=2$.

Now let us assume that $k \geqslant 3$ and the statement holds for all $k^{\prime}<k$. Consider a graph $G$ as in the statement and abbreviate $\mathcal{H}=K_{k}(G)$. Let $L$ and $S$ be the set of big and small vertices of $G$, respectively. Let $v$ be a big vertex. By Proposition 5.12 and induction, the link graph $L_{\mathcal{H}}(v)$ contains a $k$-clique. Hence $v$ is on a $(k+1)$-clique. It remains to show that $\mathcal{H}$ is tightly connected and spans all vertices.

The following two claims follow as in the proof of Lemma 5.3 with the only difference being that we apply Proposition 5.12 instead of Proposition 5.4.

Observation 5.13. If $v$ is a big vertex, then $L_{\mathcal{H}}(v)$ is tightly connected and spans $N_{G}(v)$.

Observation 5.14. If $v$ is a big vertex and $u \in N_{G}(v)$, then $u$ and $v$ belong to a common $k$-clique of $G$. 
Together with Proposition 5.12, Observation 5.13 implies that, given a big vertex $v$, all the $k$-edges containing $v$ belong to the same tight component of $\mathcal{H}$. We will denote that component by $\mathcal{H}_{v}$.

Observation 5.15. If $u, v$ are big vertices, then $\mathcal{H}_{u}=\mathcal{H}_{v}$.

Proof of the claim. It suffices to show that there is a big vertex $w$ in the common neighbourhood of $u$ and $v$. Indeed, if this is the case, then there exists a $k$-clique $X$ containing both $u$ and $w$ by Observation 5.14. Since $u$ and $w$ are big vertices, then $X$ belongs to $\mathcal{H}_{w}$ as well as $\mathcal{H}_{u}$, hence $\mathcal{H}_{u}=\mathcal{H}_{w}$. By symmetry, also $\mathcal{H}_{v}=\mathcal{H}_{w}$ and thus $\mathcal{H}_{u}=\mathcal{H}_{v}$.

Now let us show that $u$ and $v$ share a big neighbour. Any two big vertices $u$ and $v$ share stricly more than $(k-2) n / k \geqslant k-2$ neighbours (we used $n \geqslant k$ ), so $|N(u) \cap N(v)| \geqslant k-1$. If $N(u) \cap N(v)$ contains a $(k-1)$-clique $X^{\prime}$, then $X^{\prime} \cup\{u\}$ and $X^{\prime} \cup\{v\}$ are $k$-cliques in $\mathcal{H}_{u}, \mathcal{H}_{v}$ respectively, which share $k-1$ vertices, this immediately shows that $\mathcal{H}_{u}=\mathcal{H}_{v}$. Therefore, we can assume that there are two non-adjacent vertices in the joint neighbourhood of $u$ and $v$. But then one of these two vertices is big and we are done.

Observation 5.16. Every vertex of $G$ is contained in a $k$-clique.

Proof. We know already that big vertices in $L$ are covered by $k$-cliques, so it remains to consider small vertices. Let $y$ be a small vertex and let $x$ be a non-neighbour of $y$ (which exists, since $y$ is small). The Ore-type condition implies $\operatorname{deg}(x)+\operatorname{deg}(y)>2(k-1) n / k$, and $\operatorname{deg}(x)<n$ implies $\operatorname{deg}(y) \geqslant(k-2) n / k+2 \geqslant k$, since $n \geqslant k$. If there exists a big vertex in $N(y)$, we are done by Observation 5.14. Otherwise, $N(y) \subseteq S$, but since $S$ forms a clique, we deduce $y$ is contained in a $k$-clique, as desired.

By Observation 5.15, there exists a tight component $\mathcal{H}^{\prime}$ of $\mathcal{H}$ which contains $\mathcal{H}_{u}$ for every big vertex $u$. By Observation 5.16, we can finish the proof by showing that $\mathcal{H}^{\prime}$ actually contains all edges of $\mathcal{H}$. For a contradiction, suppose $X$ is an edge in $H$ that is not in $\mathcal{H}^{\prime}$. Then $X$ cannot intersect $L$, so it must be completely contained in $S$, hence $|S| \geqslant k$. By assumption, one of any two non-connected vertices in $G$ is big, hence $G[S]$ is a clique. Thus, no vertex of $L$ can have $k-1$ neighbours in $S$, otherwise it would form a $k$-clique in $H^{\prime}$ which is in the same tight component as $X$. Thus $\operatorname{deg}(x, S) \leqslant k-2$ for all $x \in L$, and thus $e(L, S) \leqslant(k-2)|L|=(k-2)(n-|S|)$.

Let $y \in S$. This vertex must have a non-neighbour $x$ (otherwise it would not be small). Since $G[S]$ is a clique, this non-neighbour $x$ must be in $L$. By assumption, $x$ and $y$ have more than $(k-2) n / k$ common neighbours, and at most $k-2$ of them can be in $S$ since $\operatorname{deg}(x, S) \leqslant k-2$. Thus $y$ has more than $(k-2) n / k-(k-2)=(k-2)(n / k-1)$ neighbours in $L$. Therefore, $\operatorname{deg}(y, L)>(k-2)(n / k-1)$ holds for every $y \in S$, and therefore $e(L, S)>$ $(k-2)(n / k-1)|S|$. Combined with the upper bound we had for $e(L, S)$, we obtain

$$
(k-2)(n / k-1)|S|<e(L, S) \leqslant(k-2)(n-|S|) .
$$

Since $k \geqslant 3$, then $k-2>0$, so dividing we get $|S|(n / k-1)<n-|S|$, which after rearranging gives $|S|<k$, a contradiction.

Since random (induced) subgraphs inherit Ore's condition, we can easily obtain the following strengthened version of Proposition 5.12.

Proposition 5.17. Let $1 / n \ll 1 / s \ll \mu, 1 / k$, and let $\ell=k s^{4 k}$. Let $G$ be $a\left(\frac{k-1}{k}+\mu\right)$ Ore graph on $n$ vertices. Then every vertex of $G$ is on at least $s^{-2 k} n^{k-1}$ many $k$-cliques. Moreover, all big vertices of $G$ are on at least $s^{-2 k} n^{k}$ many $(k+1)$-cliques. Finally, for every two $k$-cliques $X, Y$ in $G$, there are at least $s^{-2 \ell} n^{\ell}$ tight walks of length $\ell+2 k$, which start with $X$ and end with $Y$.

Proof. Consider a vertex $v \in V(G)$, a $\left(\frac{k-1}{k}+\mu\right)$-big vertex $w$ and any two $k$-cliques $X, Y$ in $G$. Select a random subset $S \subseteq V(G)$ of size precisely $s$, and let $S^{\prime}=S \cup X \cup Y \cup\{v, w\}$. By the choice of constants and using standard concentration bounds, it follows that with probability at least $1 / 2$ the graph $G\left[S^{\prime}\right]$ is $\frac{k-1}{k}$-Ore, and also $w$ is $\frac{k-1}{k}$-big in $G\left[S^{\prime}\right]$; say such 
a choice of $S$ is successful. If $S$ is successful, by Lemma 5.11 applied to $G\left[S^{\prime}\right]$, it follows that $K_{k}\left(G\left[S^{\prime}\right]\right)$ is tightly connected, every vertex is on a $k$-clique in $G\left[S^{\prime}\right]$ and every $\frac{k-1}{k}$-big vertex is on a $(k+1)$-clique in $G\left[S^{\prime}\right]$. Those properties also imply that there exists a tight walk $W$ from $X$ to $Y$ of length precisely $\ell$ in $G\left[S^{\prime}\right]$ (this can be seen, e.g. by applying Proposition 7.4, to be proven later).

Using the previous discussion, now we can show the desired statements. Let us prove first that every vertex is on at least $s^{-2 k} n^{k-1}$ ordered cliques. For each successful $S$, we have shown $G\left[S^{\prime}\right]$ contains a $k$-clique which contains $v$. There are at least $\frac{1}{2}\left(\begin{array}{l}n \\ s\end{array}\right)$ successful choices of $S$, and each $(k-1)$-clique (after removing $v$ ) is contained at most in $\left(\begin{array}{l}n-k-1 \\ s-k-1\end{array}\right)$ successful $S$. Thus the number of $k$-cliques which contain $v$ must be at least $\frac{1}{2}\left(\begin{array}{l}n \\ s\end{array}\right)\left(\begin{array}{c}n-k-1 \\ s-k-1\end{array}\right)^{-1} \geqslant s^{-2 k} n^{k-1}$, as required. The other properties follow similarly.

Now Proposition 5.17 implies Lemma 5.9 since the strengthened properties survive in every $\nu$-approximation if $\nu$ is sufficiently small. Next, we turn to the proof of Lemma 5.10.

Lemma 5.18. Let $1 / n \ll \nu \ll \beta, \mu, 1 / k$ with $n$ divisible by $k$. Let $G$ be $a\left(\frac{k-1}{k}+\mu\right)$-Ore graph on $n$ vertices. Let $G^{\prime}$ be a $\nu$-approximation of $G$ on $n$ vertices. Then $K_{k}\left(G^{\prime}\right)$ has a matching which covers all but at most $\beta n$ vertices.

Proof. By Theorem 2.3, $G$ has at least one $k$-clique factor. Let $\nu \ll 1 / C \ll \beta, \mu, 1 / k$. Suppose first that

for each edge $e \in G$, and a perfect $k$-clique factor $M$ of $G$ chosen uniformly at random, $e$ appears in $M$ with probability at most $C / n$.

By the choice of constants we can assume $\nu \leqslant \beta /(C k)$. Choose a $k$-clique factor $M^{\prime}$ of $G$ uniformly at random. By property (5.2), the expected number of edges not in $G^{\prime}$ which are used in $M^{\prime}$ is at most $e\left(G \backslash G^{\prime}\right) C / n \leqslant \beta n / k$. Thus there exists some $k$-clique factor $M$ of $G$ which uses at most $\beta n / k$ edges which are not in $G^{\prime}$. Removing the cliques in $M$ that are not in $G^{\prime}$ uncovers at most $\beta n$ vertices, so this translates to a matching in $K_{k}\left(G^{\prime}\right)$ that leaves at most $\beta n$ uncovered vertices, as required.

We can show property (5.2) using a 'switching' argument, closely following the arguments by Glock, Gould, Joos, Kühn and Osthus [29, Section 4]. Fix $e \in E(G)$. Let $\mathcal{M}$ be the set of all $k$-clique factors in $G$, and let $\mathcal{M}_{e} \subseteq \mathcal{M}$ be the $k$-clique factors which use $e$. The probability that $e$ appears in $M$ among a uniformly chosen $k$-clique factor is then precisely $\left|\mathcal{M}_{e}\right| /|\mathcal{M}|$. Note that $\left|\mathcal{M}_{e}\right| /|\mathcal{M}| \leqslant\left|\mathcal{M}_{e}\right| /\left|\mathcal{M} \backslash \mathcal{M}_{e}\right|$.

Given $M \in \mathcal{M}_{e}$, an $(e, M)$-switching is a $k$-clique matching $Y$ in $G$ satisfying

(i) the vertices of $e$ are covered by exactly two cliques in $Y$, and

(ii) for all $K \in M$, either $V(K) \subseteq V(Y)$ or $V(K) \cap V(Y)=\emptyset$.

The key observation is that if $M \in \mathcal{M}_{e}$ and $Y$ is an $(e, M)$-switching, then we can obtain a new perfect matching $M^{\prime} \in \mathcal{M} \backslash \mathcal{M}_{e}$ from $M$ by substituting $M[V(Y)]$ with $Y$.

Choose $1 / C \ll 1 / m \ll \mu, \beta, 1 / k$. Define an auxiliary bipartite multigraph $B_{m}$ with bipartition $\left(\mathcal{M}_{e}, \mathcal{M} \backslash \mathcal{M}_{e}\right)$. For each $M \in \mathcal{M}_{e}$ and each $(e, M)$-switching $Y$ on exactly $m$ cliques, add an edge in $B_{m}$ from $M$ to the matching $M^{\prime} \in \mathcal{M} \backslash \mathcal{M}_{e}$ obtained by replacing $M[V(Y)]$ with $Y$. Let $\Delta_{m}$ be the maximum degree in $B_{m}$ over all $M^{\prime} \in \mathcal{M} \backslash \mathcal{M}_{e}$, and let $\delta_{m}$ be the minimum degree in $B_{m}$ over all $M \in \mathcal{M}_{e}$. Double-counting $\left|E\left(B_{m}\right)\right|$, we get that $\left|\mathcal{M}_{e}\right| /\left|\mathcal{M} \backslash \mathcal{M}_{e}\right| \leqslant \Delta_{m} / \delta_{m}$. To conclude, we upper bound $\Delta_{m}$ and lower bound $\delta_{m}$.

To bound $\Delta_{m}$, fix $M^{\prime} \in \mathcal{M} \backslash \mathcal{M}_{e}$. We bound the number of pairs $(M, Y)$ where $M \in \mathcal{M}$ and $Y$ is an $(e, M)$-switching with $m$ cliques which produces $M^{\prime}$. Any such $Y$ must contain the two $k$-cliques in $M^{\prime}$ which cover $e$, and precisely $m-2$ other cliques of $M^{\prime}$, which can be chosen in at most $n^{m-2}$ ways. Having set $V(Y)$, the number of valid choices for $M[V(Y)]$ (and thus $M$ ) is at most $(\mathrm{km}) !$. Thus we get $\Delta_{m} \leqslant(\mathrm{~km}) ! n^{m-2}$.

To bound $\delta_{m}$, fix $M \in \mathcal{M}_{e}$, and we bound the number of $(e, M)$-switchings from below. Let $K \in M$ be the $k$-clique containing $e$. Any $(e, M)$-switching $Y$ must cover all of $V(K)$ and also the vertex set of other $m-1$ cliques of $M$. There are $\left(\begin{array}{c}n / k-1 \\ m-1\end{array}\right)$ choices for the remaining $m-1$ cliques in $M$ apart from $K$, which determine $V(Y)$ completely. Using 
$1 / n \leqslant 1 / m \leqslant \mu, 1 / k$, a probabilistic argument (see [29, Appendix] for detailed calculations in a similar setup) shows that if we select $m-1$ distinct cliques $K_{1}, \ldots, K_{m-1}$ from $M \backslash K$ at random, with probability at least $1-e^{-\sqrt{m-1}}$ it holds that $G\left[V\left(K \cup K_{1} \cup \cdots \cup K_{m-1}\right)\right]$ is $\left(\frac{k-1}{k}+\frac{\mu}{2}\right)$-Ore. Thus there are at least $\left(1-e^{-\sqrt{m-1}}\right)\left(\begin{array}{c}n / k-1 \\ m-1\end{array}\right) \geqslant(m k)^{-m} n^{m-1}$ such choices. For each such choice, let $V=V\left(K \cup K_{1} \cup \cdots \cup K_{m-1}\right)$, choose precisely two cliques $K^{\prime}, K^{\prime \prime}$ in $V$ which cover $e$, and choose a perfect matching $Y^{\prime}$ in $V \backslash V\left(K^{\prime} \cup K^{\prime \prime}\right)$ (which exists since $G[V]$ is $\left(\frac{k-1}{k}+\frac{\mu}{2}\right)$-Ore. Then $Y=\left\{K^{\prime}, K^{\prime \prime}\right\} \cup Y^{\prime}$ is an $(e, M)$-switching with exactly $m$ cliques corresponding uniquely to this choice. We deduce that $\delta_{m} \geqslant(m k)^{-m} n^{m-1}$.

Putting everything together, we have that $\left|\mathcal{M}_{e}\right| /|\mathcal{M}| \leqslant\left|\mathcal{M}_{e}\right| /\left|\mathcal{M} \backslash \mathcal{M}_{e}\right| \leqslant \Delta_{m} / \delta_{m} \leqslant$ $(k m) ! n^{m-2}(m k)^{m} /\left(n^{m-1}\right) \leqslant C / n$, which finally proves property (5.2).

Proof of Lemma 5.10. Let $1 / n \ll \nu \ll \beta \ll \nu^{\prime} \ll \mu, 1 / k$, where $\beta$ comes from Lemma 5.18 and $\nu^{\prime}$ from Lemma 5.9. Let $G$ be a $\left(\frac{k-1}{k}+\mu\right)$-Ore graph on $n$ vertices, and let $G^{\prime}$ be a $\nu$-approximation of $G$.

By Lemma 5.9 and $\nu \ll \nu^{\prime}$, every vertex of $G$ is on at least $\nu^{\prime} n^{k-1} k$-cliques. If $G$ has fewer than $\nu^{\prime 2} n$ small vertices, we match all small vertices with vertex-disjoint $k$-cliques, and remove these cliques from the graph. The proof then continues in the same way. Hence in the following, we will assume that $G$ has at least $\nu^{\prime 2} n$ small vertices. Since small vertices form a clique in $G$, all small vertices are on at least $\nu^{\prime 3} n^{k}(k+1)$-cliques. By Lemma 5.9, the same holds for big vertices. Since $\nu \ll \beta \ll \nu^{\prime}$, it follows that every vertex in $G$ is on at least $\sqrt{\beta} n^{k}(k+1)$-cliques.

A standard concentration argument then allows us to select set of at most $\sqrt{\beta} n k$-cliques $A$, such that every vertex of $G$ has at least $\beta n$ of these cliques in its neighbourhood. Adding at most $k-1$ further cliques to $A$, we can ensure that $n-v(A)$ is divisible by $k$. We then apply Lemma 5.18 to $G^{\prime \prime}=G\left[V\left(G^{\prime}\right) \backslash V(A)\right], F=E\left(G^{\prime \prime}\right) \backslash E\left(G^{\prime}\right)$ to cover all but $\beta n$ vertices of $G^{\prime}-V(A)$ with disjoint $k$-cliques. Finally, we absorb the remaining vertices into $A$ by adding each remaining vertex on a private $(k+1)$-clique and giving each involved $k$-clique weight $1 / k$. This results in a perfect fractional matching of $K_{k}\left(G^{\prime}\right)$.

5.4. Uniformly dense and inseparable graphs. In this section, we prove Theorem 2.7. In light of Theorem 3.5, it suffices to show that any inseparable and locally dense graph admits a robust Hamilton framework, and this is precisely the content of Lemma 5.19. We introduce some further definitions to describe the statement.

For a $k$-graph $\mathcal{H}$ on $n$ vertices and $\eta>0$, let $\partial_{\eta} \mathcal{H}=\left\{X \in E\left(\partial_{k-1} \mathcal{H}\right): \operatorname{deg}_{\mathcal{H}}(X) \geqslant \eta n\right\}$ be the set of shadow edges of degree at least $\eta n$ in $\mathcal{H}$. We define the $\eta$-adherence of $\mathcal{H}$ to be the spanning subgraph $A_{\eta}(\mathcal{H}) \subseteq \mathcal{H}$ that consists of the edges of $\mathcal{H}$ that contain an element of $\partial_{\eta} \mathcal{H}$.

Lemma 5.19. Let $1 / n \ll \varrho \ll \mu^{\prime} \ll \eta \ll \mu, d, 1 / k$. Suppose $G$ is a $\mu$-inseparable $(\varrho, d)$-dense graph on $n \geqslant n_{0}$ vertices, and let $\mathcal{H}=A_{\eta}\left(K_{k}(G)\right)$. Then $(G, \mathcal{H})$ is a $\mu^{\prime}$-robust Hamilton framework.

The connectivity part of the proof of Lemma 5.19 relies on the following result of Ebsen, Maesaka, Reiher, Schacht and Schülke [22], which we restate in our notation.

Lemma 5.20 ([22, Connecting Lemma, Lemma 3.1]). Let $1 / n, 1 / M, \varrho, \zeta \ll d, \mu, \eta, 1 / k$. Let $G$ be $a(\varrho, d)$-dense and $\mu$-inseparable graph on $n$ vertices. Let $X_{1}, X_{2} \in V(G)^{k-1}$ be disjoint tuples whose unordered sets are in $\partial_{\eta}\left(K_{k}(G)\right)$. Then the number of tight $\left(X_{1}, X_{2}\right)$-paths in $K_{k}(G)$ of length $m$ is at least $\zeta n^{m-2(k-1)}$ for some $2(k-1)+1 \leqslant m \leqslant M$.

Corollary 5.21. Let $1 / n \ll \mu^{\prime} \ll \varrho \ll d, \mu, \eta, 1 / k$. Let $G$ be a $(\varrho, d)$-dense and $\mu$-inseparable graph on $n$ vertices and $\mathcal{H}=A_{\eta}\left(K_{k}(G)\right)$. Let $G^{\prime} \subseteq G$ be a $\left(\mu^{\prime}, \mu^{\prime}\right)$-approximation of $G$ and, let $\mathcal{H}^{\prime}=\mathcal{H} \cap K_{k}\left(G^{\prime}\right)$. Then $\mathcal{H}^{\prime}$ is contained in a tight component of $K_{k}\left(G^{\prime}\right)$.

Proof. Let $M, \zeta$ be such that $\mu^{\prime} \ll 1 / M, \zeta \ll d, \mu, 1 / k$. Consider any two disjoint edges $Y_{1}, Y_{2}$ in $\mathcal{H}^{\prime}$. By assumption, there are $X_{1} \subseteq Y_{1}, X_{2} \subseteq Y_{2}$ with $X_{1}, X_{2} \in \partial_{\eta}(\mathcal{H})$. It follows by Lemma 5.20 that the number of tight $\left(X_{1}, X_{2}\right)$-paths in $K_{k}(G)$ of length $m$ is at least 
$\zeta n^{m-2(k-1)}$ for some $2(k-1)+1 \leqslant m \leqslant M$. Since $1 / n \ll \mu^{\prime} \ll \zeta, 1 / M$, at least one of these paths 'survives' the transition from $K_{k}(G)$ to $K_{k}\left(G^{\prime}\right)$. Hence $Y_{1}$ and $Y_{2}$ are in the same tight component of $K_{k}\left(G^{\prime}\right)$. The case where $Y_{1}, Y_{2}$ intersect follows by transitivity.

We also need the following facts, which are a simple consequence of $(\varrho, d)$-density.

Lemma 5.22 ([22, Lemma 2.1]). Let $k \geqslant 1, d \in[0,1]$ and $\varrho>0$. Every $(\varrho, d)$-dense graph

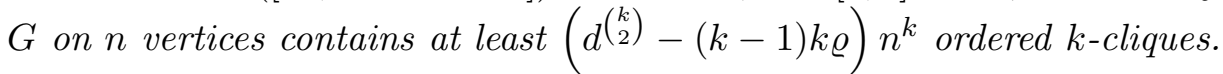

Corollary 5.23. Let $\varrho \leqslant \eta \ll d, k$. Let $G$ be a $(\varrho, d)$-dense graph $G$ on $n$ vertices, and let $\mathcal{H}=A_{\eta}\left(K_{k}(G)\right)$. Then $\mathcal{H}[S]$ has at least $\eta n^{k}$ edges for every $S \subseteq V(G)$ of size at least $\eta^{1 /(2 k)} n$.

Proof. Let $F=G[S]$ and $m=|S|$. Observe that $F$ is $(\sqrt{\varrho}, d)$-dense. This follows, since for any $U \subseteq V(F)$, we have $e(U) \geqslant d|U|^{2} / 2-\varrho n^{2}$ and, we also have $\varrho n^{2} \leqslant \sqrt{\varrho} m^{2}$ since $m \geqslant \eta^{1 /(2 k)} n$ and $\varrho \ll \eta$.

By Lemma 5.22, the number of edges in $K_{k}(F)$ is at least

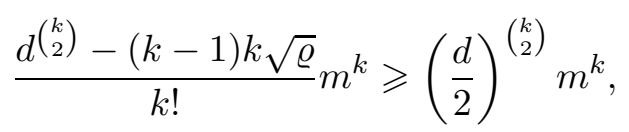

where we used that $\varrho \ll d, k$ in the last inequality. We define a subgraph $\mathcal{J} \subseteq K_{k}(F)$ as follows. Initially, $\mathcal{J}=K_{k}(F)$. Next, we iteratively delete edges from $\mathcal{J}$, at each step removing those edges that are contained in a $(k-1)$-set that is contained in at most $\sqrt{\eta} m$ edges (in the updated hypergraph). So the number of removed edges is at most

$$
\sqrt{\eta} m\left(\begin{array}{c}
m \\
k-1
\end{array}\right) \leqslant \frac{\sqrt{\eta}}{(k-1) !} m^{k} .
$$

Since $\eta \ll d, k$ it follows that, after the removal, the number of edges in $\mathcal{J}$ is at least

$$
\left(\frac{d}{2}\right)^{\left(\begin{array}{c}
k \\
2
\end{array}\right)} m^{k}-\frac{\sqrt{\eta}}{(k-1) !} m^{k} \geqslant \frac{1}{2}\left(\frac{d}{2}\right)^{\left(\begin{array}{c}
k \\
2
\end{array}\right)} m^{k} \geqslant \frac{1}{2}\left(\frac{d}{2}\right)^{\left(\begin{array}{c}
k \\
2
\end{array}\right)} \eta^{1 / 2} n^{k} \geqslant \eta n^{k},
$$

where in the second to last inequality we used $m \geqslant \eta^{1 /(2 k)} n$ and in the last inequality we used $\eta \ll d$. Each of these edges induces a $k$-clique $X$ in $F$, such that every $(k-1)$-set of $X$ is in at least $\sqrt{\eta} m$ edges of $\mathcal{J}$. In particular, each such $(k-1)$-set must be in at least $\sqrt{\eta} m \geqslant \eta n$ $k$-cliques of size $k$ in $K_{k}(F)$, and thus in $\mathcal{H}=\partial_{\eta}\left(K_{k}(G)\right)$. Thus $e(\mathcal{H}[S]) \geqslant e(\mathcal{J}) \geqslant \eta n^{k}$, as required.

Proposition 5.24. Let $1 / n \ll \mu^{\prime} \ll \varrho \ll \eta \ll d, \mu, 1 / k$. Let $G$ be $a(\varrho, d)$-dense and $\mu$ inseparable graph on $n$ vertices and $\mathcal{H}=A_{\eta}\left(K_{k}(G)\right)$. Let $G^{\prime} \subseteq G$ be a $\left(\mu^{\prime}, \mu^{\prime}\right)$-approximation of $G$, and let $\mathcal{H}^{\prime}=\mathcal{H} \cap K_{k}\left(G^{\prime}\right)$. Then $\mathcal{H}^{\prime}$ contains a perfect fractional matching.

Proof. Let $\eta^{\prime}>0$ with $\eta \ll \eta^{\prime} \ll d, \mu, 1 / k$. Consider a vertex $v \in V\left(\mathcal{H}^{\prime}\right)$. Since $G$ is $\mu$ inseparable, we have $\left|N_{G}(v)\right| \geqslant \mu n \geqslant\left(\eta^{\prime}\right)^{1 /(2 k)} n$, where the last inequality follows by our choice of $\eta^{\prime}$. Since $A_{\eta^{\prime}}\left(K_{k}(G)\right) \subseteq A_{\eta}\left(K_{k}(G)\right)=\mathcal{H}$, an application of Corollary 5.23 with $\eta^{\prime}$ in place of $\eta$ implies that there are at least $\eta^{\prime} n^{k}$ edges in $\mathcal{H}\left[N_{G}(v)\right]$. Since $1 / n \ll \mu^{\prime} \ll \eta^{\prime}$, at least $\left(\eta^{\prime} / 2\right) n^{k}$ of these edges are still contained in $\mathcal{H}^{\prime}\left[N_{G^{\prime}}(v)\right]$. By a standard application of Chernoff's inequality [36, Theorem 2.1], we can find a matching $A \subseteq \mathcal{H}^{\prime}$ of size at most $\eta^{\prime} n$ such that $A$ has at least $\eta^{1 /(2 k)} n$ edges in the neighbourhood $N_{G^{\prime}}(v)$ of every vertex $v \in V\left(\mathcal{H}^{\prime}\right)$. Next, we find a matching $M \subseteq \mathcal{H}^{\prime}-V(A)$ such that $M \cup A$ covers all but $\eta^{1 /(2 k)} n$ vertices of $\mathcal{H}^{\prime}$. We proceed greedily, using Corollary 5.23 (this time using $\eta$ as input) to find an edge in the set of uncovered vertices in each step. We then join each of the remaining vertices to a (private) edge of $A$. This gives a collection of pairwise disjoint edges and $(k+1)$-cliques in $\mathcal{H}^{\prime}$ that together cover all vertices of $\mathcal{H}^{\prime}$. Thus, we can define a perfect fractional matching by assigning weight 1 to single edges and weight $1 / k$ to the edges of the $(k+1)$-cliques. 
Proof of Lemma 5.19. Let $G^{\prime} \subseteq G$ be an arbitrary $\left(\mu^{\prime}, \mu^{\prime}\right)$-approximation of $G$ and, let $\mathcal{H}^{\prime}=$ $\mathcal{H} \cap K_{k}\left(G^{\prime}\right)$. By Corollary 5.21, $\mathcal{H}^{\prime}$ is contained in a tight component of $K_{k}\left(G^{\prime}\right)$. By Proposition 5.24, $\mathcal{H}^{\prime}$ has a perfect fractional matching. Consider any vertex $v \in V\left(\mathcal{H}^{\prime}\right)$. Since $G$ is $\mu$-inseparable, we have $\left|N_{G}(v)\right| \geqslant \mu n \geqslant \eta n$. By Corollary 5.23, there are at least $\eta n^{k}$ edges in $\mathcal{H}\left[N_{G}(v)\right]$. So in particular, every vertex in $\mathcal{H}$ has at least $\eta n^{k} \geqslant \mu^{\prime} n^{k}$ linked edges. Moreover, since $\mu^{\prime} \ll \eta$, one of these sets $Y$ together with $v$ induces a $(k+1)$-clique in $G^{\prime}$. Hence $\mathcal{H}^{\prime}$ contains a $(k+1)$-clique.

5.5. Deficiency. To show Theorem 2.9, we will need some preparations. Consider the function given by

$$
f(n, t, k)=\left\{\begin{array}{ll}
\left(\begin{array}{l}
n \\
2
\end{array}\right)-\left(\begin{array}{c}
\frac{t}{k-1} \\
2
\end{array}\right)-\frac{t}{k-1}\left(n-\frac{t}{k-1}\right) & \text { if } t<\frac{(k-1) n}{2 k^{2}-2 k+1}, \\
\left(\begin{array}{l}
n \\
2
\end{array}\right)-\left(\begin{array}{c}
\frac{n+t}{k} \\
2
\end{array}\right) & \text { if } \frac{(k-1) n}{2 k^{2}-2 k+1} \leqslant t<(k-1) n, \text { and } \\
0 & \text { if } t \geqslant(k-1) n .
\end{array} .\right.
$$

It is not difficult to check that, for fixed $k$, the function $g(n, t, k)$ appearing in Theorem 2.8 satisfies $f(n, t, k)=g(n, t, k)+o\left(n^{2}\right)$. We will find it more convenient to work with $f(n, t, k)$ instead.

We show next that, to show Theorem 2.9, we can assume $t$ is not very close to 0 .

Proposition 5.25. Let $\mu \geqslant 0$ and $n, k, t$ integers with $k \geqslant 2$ and $t \leqslant k n$. Suppose $G$ is a graph on $n$ vertices with $e(G)>f(n, t, k)+\mu n^{2}$. Then $t \geqslant \mu(n+t) / 3$.

Proof. We have $\mu n^{2}+f(n, k, t)<e(G) \leqslant\left(\begin{array}{c}n \\ 2\end{array}\right)$. Let $\alpha n=t /(k-1)$. For $t<(k-1) n /\left(2 k^{2}-\right.$ $2 k+1)$, we obtain

$$
\mu n^{2} \leqslant\left(\begin{array}{c}
\alpha n \\
2
\end{array}\right)+\alpha n(n-\alpha n) \leqslant \frac{1}{2} \alpha^{2} n^{2}+\alpha n(n-\alpha n) .
$$

Hence $\alpha^{2}+2 \alpha(1-\alpha) \geqslant 2 \mu$, which implies $\alpha \geqslant \mu$. This gives $t \geqslant \mu(k-1) n$. We can conclude, since $t \leqslant k n$ and $k \geqslant 2$ imply that $\mu(k-1) n \geqslant \mu(n+t) / 3$.

Again, we have to be careful since deleting a few edges can destroy the structure of $G * K_{t}$, so Theorem 2.8 cannot be applied directly to approximations of $G * K_{t}$ to get Hamilton frameworks. However, this is merely a technical obstacle as the next proposition shows.

Proposition 5.26. Let $1 / n \ll \nu \ll \mu, 1 / k$ and $k \geqslant 3$. Let $t \leqslant k n$. Suppose $G$ is a graph on $n$ vertices with $e(G) \geqslant f(n, t, k)+\mu n^{2}$. Let $F$ be a $\nu$-approximation of $G * K_{t}$. Then $\mathcal{H}^{\prime}=K_{k}(F)$ has a fractional perfect matching.

Proof. It will be convenient to assume that $\mu \ll 1$. We set $\mathcal{H}^{\prime}=K_{k}(F), K^{\prime}=V(F) \cap V\left(K_{t}\right)$, and $t^{\prime}=\left|K^{\prime}\right|$. We begin by showing that there is an 'absorbing' structure $A \subseteq \mathcal{H}^{\prime}\left[K^{\prime}\right]$. First, by Proposition 5.25, we can assume that $t \geqslant \mu(n+t) / 4$. This implies that $\nu(n+t) \leqslant$ $4 \nu \mu^{-1} t \leqslant \mu t$. Next, fix a vertex $v \in V(F)$. Since $F$ is a $\nu$-approximation and $\nu \ll \mu$, we have $\operatorname{deg}_{F}\left(v ; K^{\prime}\right) \geqslant t-\nu(n+t) \geqslant(1-\mu) t^{\prime}$. Similarly, $F\left[N_{F}\left(v ; K^{\prime}\right)\right]$ has minimum degree at least $(1-2 \mu) t^{\prime}$, as vertices in $K^{\prime}$ have lost at most $\nu(n+t)$ neighbours each. It follows that $F\left[N_{F}\left(v ; K^{\prime}\right)\right]$ has at least $(1-4 \mu)\left(\begin{array}{c}t^{\prime} \\ k\end{array}\right)$ many $k$-cliques. A standard probabilistic argument (choose each $k$-set in $F\left[K^{\prime}\right]$ with probability $p=k ! \mu^{2} t^{\prime 1-k} / 2$, delete intersecting copies) yields the existence of a matching $A \subseteq \mathcal{H}^{\prime}\left[K^{\prime}\right]$ of size at most $\mu^{2} t^{\prime} \leqslant \mu^{2} t$ with the additional property that, for every vertex $v \in V(F)$ there are at least $\mu^{2} t^{\prime} / 8 \geqslant \mu^{3}(n+t) / 64$ edges $X \in E(A)$ such that $\{v\} \cup X$ is a $(k+1)$-clique in $F$.

Next, we find a large matching in $\mathcal{H}^{\prime}-V(A)$. Let $t^{\prime \prime}=t^{\prime}-V(A)$. By choice of $A$, we have $t^{\prime \prime} \geqslant\left(1-2 \mu^{2}\right) t^{\prime} \geqslant(1-2 \mu) t$. Set $G^{\prime}=F \cap G$ and $n^{\prime}=v(F \cap G)$. Since $F$ is a $\nu$-approximation, we have $n^{\prime} \geqslant n-(n+t) \geqslant(1-2 k \nu) n$. Moreover, $e\left(G^{\prime}\right) \geqslant f(n, t, k)+\mu n^{2}-\nu(n+t)^{2}$. Using these bounds together with $1 / n \ll \nu \ll \mu, 1 / k$ a tedious but straightforward calculation yields that $e\left(G^{\prime}\right) \geqslant f\left(n^{\prime}, t^{\prime \prime}, k\right)+(\mu / 2) n^{\prime 2}$. 
Now define $F^{\prime}=G^{\prime} * K_{t^{\prime \prime}}$. Since $e\left(G^{\prime}\right) \geqslant f\left(n^{\prime}, t^{\prime \prime}, k\right)+(\mu / 2) n^{\prime 2}$, we can apply Theorem 2.8 (Clique factors under deficiency conditions) to obtain a $K_{k}$-clique matching $M$ in $F^{\prime}$ which misses at most $k-1$ vertices of $F^{\prime}$. We will use $M$ to find a large $K_{k}$-matching in $F$.

Let $\phi: V\left(F^{\prime}\right) \rightarrow V(F)$ be defined as follows, set $\phi(x)=x$ for all $x \in V\left(G^{\prime}\right)$, and consider a random bijection $\phi: V\left(K_{t^{\prime \prime}}\right) \rightarrow V\left(K^{\prime}-V(A)\right)$. Let $\phi(M)$ be the (random) image of $M$ in $F$ via $\phi$. Let $x \in V\left(F^{\prime}\right)$ and $y \in V\left(K_{t^{\prime \prime}}\right)$. Since $F$ is a $\nu$-approximation, $\phi(x)$ misses at most $\nu(n+t)$ of its possible $t^{\prime \prime}$ neighbours in $F$ (or $t^{\prime \prime}-1$ if $x$ is in $K_{t^{\prime \prime}}$ ). So the probability that $\phi(x) \phi(y)$ is not an edge in $F$ is at most $\nu(n+t) / t^{\prime \prime} \leqslant \nu /(\mu / 2) \leqslant \nu^{3 / 4}$, where the inequality follows from $\nu \ll \mu$. Hence, the expected number of edges of $M$ which are mapped to nonedges of $F$ via $\phi$ is at most $\nu^{3 / 4} e(M) \leqslant \nu^{3 / 4}\left(\begin{array}{l}k \\ 2\end{array}\right)\left(n^{\prime}+t^{\prime \prime}\right) / k \leqslant \nu^{2 / 3}\left(n^{\prime}+t^{\prime \prime}\right)$. It follows that there exists a choice of $\phi$ where at most $\nu^{2 / 3}\left(n^{\prime}+t^{\prime \prime}\right)$ edges of $M$ are mapped to non-edges. Recall that $v(M) \geqslant n^{\prime}+t^{\prime \prime}-k+1$. Note that removing a $k$-clique which contains a non-edge uncovers $k$ vertices. It follows that $\mathcal{H}^{\prime}-V(A)$ contains a matching which covers all but at most $k-1+k \nu^{2 / 3}\left(n^{\prime}+t^{\prime \prime}\right) \leqslant \nu^{1 / 2}\left(n^{\prime}+t^{\prime \prime}\right)$ vertices of $\mathcal{H}^{\prime}$.

To finish, we cover the vertices of $\mathcal{H}^{\prime}-V(A)$ by joining each of the remaining vertices to a (private) edge of $A$. This is possible, since each vertex has $\mu^{3}(n+t) / 64$ edges available in $A$ and the number of uncovered vertices is certainly at most $\nu^{1 / 2}\left(n^{\prime}+t^{\prime \prime}\right) \leqslant \mu^{3}(n+t) / 64$. This gives a collection of pairwise disjoint edges and $(k+1)$-cliques in $\mathcal{H}^{\prime}$ that together cover all vertices of $\mathcal{H}^{\prime}$. Thus, we can define a perfect fractional matching by assigning weight 1 to single edges and weight $1 / k$ to the edges of the $(k+1)$-cliques.

Proof of Theorem 2.9. Let $n_{2}, \nu$ be given by Proposition 5.26 under input $k, \mu$. Without loss of generality we can also suppose $\nu \leqslant \mu /(3(k+2))$. Let $\mu^{\prime}, n_{1}$ be given by Proposition 3.6 under input $k, \nu$. Let $\beta, z>0$, and $n_{3} \in \mathbb{N}$ be obtained from Theorem 3.5 applied with $k, \Delta, \mu^{\prime}$, and let $n_{0}=\max \left\{n_{1}, n_{2}, n_{3}, k^{3} \mu^{\prime-3} \nu^{-1}\right\}$.

Let $n \geqslant n_{0}$. Given $t \geqslant 0$, it suffices to consider a graph $G$ on $n$ vertices with at least $e(G) \geqslant g(n, k, t)+2 \mu n^{2}$. Let $\mathcal{H}=K_{k}\left(G * K_{t}\right)$. By Theorem 3.5 it is enough to show that $(G, \mathcal{H})$ is a $\mu^{\prime}$-robust zero-free Hamilton framework. By Proposition 3.6 and the choice of $\mu^{\prime}, n_{0}$, it is enough to show that $\left(G * K_{t}, \mathcal{H}\right)$ is a $\nu$-proto-robust zero-free Hamilton framework.

If $t \geqslant k n$, actually there is nothing to show because $G * K_{t}$ contains every $(k+1)$-colourable graph $J$ on $n+t$ vertices: the largest colour class in $J$ has at least $(n+t) /(k+1) \geqslant n$ vertices, so we can embed $J$ in $G * K_{t}$ making sure that the largest colour class covers all of $V(G)$ and allocate the rest arbitrarily. Thus we can assume $t<k n$. By the choice of $n_{0}$, we have $e(G)>f(n, k, t)+\mu n^{2}$. Thus by Proposition 5.25, $t \geqslant \mu(n+t) / 3$. In summary, we have $\mu(n+t) / 3 \leqslant t<k n$.

Now we show that $\left(G * K_{t}, \mathcal{H}\right)$ is a $\nu$-proto-robust zero-free Hamilton framework. To do so, let $F \subseteq G * K_{t}$ be a $\nu$-approximation of $G * K_{t}$. Note that $\mathcal{H} \cap K_{k}(F)=K_{k}(F)$. We will be done if we show $K_{k}(F)$ is a zero-free Hamilton framework, that is, we need to show $K_{k}(F)$ is tightly connected, contains a perfect fractional matching, and a $(k+1)$-clique.

We show first that $K_{k}(F)$ is tightly connected. Let $L=V\left(G * K_{t}\right) \backslash V(G)$, so $|L|=t$. Let $L^{\prime}=L \cap V(F)$. Since $t \geqslant \mu(n+t) / 3 \geqslant k$, we deduce that $F\left[L^{\prime}\right]$ has minimum degree at least $\left|L^{\prime}\right|-1-\nu(n+t)>(k-1)\left|L^{\prime}\right| / k$. It is not hard to see that this implies that $K_{k}\left(F\left[L^{\prime}\right]\right)$ is tightly connected. (Formally, one can also apply Lemma 5.3.) Given this, to show $K_{k}(F)$ is tightly connected it is enough to show that for each edge $X$ in $K_{k}(F)$ is in the same tight component as the edges of $K_{k}\left(F\left[L^{\prime}\right]\right)$. We show this latter statement by induction on $|X \cap V(G)|$. If $|X \cap V(G)|=0$, then $X \subseteq L$, thus $X \in F\left[L^{\prime}\right]$, so there is nothing to show. Suppose $|X \cap V(G)|>0$, and let $X=\left\{v_{1}, \ldots, v_{k}\right\}$, ordered so that vertices in $L$ come first. In $G * K_{t}$, the common neighbourhood of $\left\{v_{1}, \ldots, v_{k-1}\right\}$ in $L$ has size at least $|L|=t$, thus in $F$ it has size at least $t-k \nu(n+t)>0$. Thus there exists $v_{0} \in L^{\prime} \cap N_{F}\left(v_{1}\right) \cap \cdots \cap N_{F}\left(v_{k-1}\right)$, so $X^{\prime}=\left\{v_{0}, v_{1}, \ldots, v_{k-1}\right\}$ is a $k$-clique in $\mathcal{H}$ such that $\left|X^{\prime} \cap V(G)\right|<|X \cap V(G)|$. Moreover, by Proposition 5.1, $X^{\prime}$ is in the same tight component as $X$. By induction hypothesis, $X^{\prime}$ is tightly connected to some $Y \in K_{k}\left(F\left[L^{\prime}\right]\right)$ and so is $X$, as required.

Next, we show $K_{k}(F)$ has a $(k+1)$-clique. This holds simply because $F\left[L^{\prime}\right]$ has minimum degree strictly larger than $(k-1)\left|L^{\prime}\right| / k$, so we can find such a clique greedily in $L^{\prime}$. Finally, 
Proposition 5.26 implies that $K_{k}(F)$ has a perfect fractional matching. So $K_{k}(F)$ is indeed a zero-free Hamilton framework, as required, and this finishes the proof.

5.6. Multipartite graphs. In this section, we show Theorem 2.11 (Multipartite Bandwidth Theorem). We start with the following simple observation.

Observation 5.27. For $r \geqslant k \geqslant 2$, let $\mathcal{U}=\left\{U_{j}\right\}_{j=1}^{r}$ be a family of $r$ disjoint sets of size $n$ each. Let $G$ be a U-partite graph with $\delta(G ; \mathcal{U})>\frac{k-1}{k} n$. Let $S$ be any set of at most $k$ vertices disjoint of some cluster $U_{j}$. Then the vertices of $S$ have a common neighbour in $U_{j}$.

Proof. The common neighbourhood of $S$ in $U_{j}$ has size at least $|S| \delta(G ; \mathcal{U})-(|S|-1) n>0$.

Next, we show a result about connectivity in multipartite graphs.

Lemma 5.28. Let $r \geqslant k \geqslant 2$, and let $G$ be a balanced $r$-partite graph on rn vertices with $\delta_{r}(G)>\frac{k-1}{k} n$. Then $K_{k}(G)$ is tightly connected.

Proof. Suppose that $G$ is $\mathcal{U}$-partite with $\mathcal{U}=\left\{U_{j}\right\}_{j=1}^{r}$. We begin by proving the case $r=k$ via induction on $k$. For $k=2$, the condition says that $\delta(G ; \mathcal{U})>n / 2$. This implies that any two vertices in a given cluster share a neighbour, which is clearly enough to conclude.

Now suppose $k>2$ is given and the result is true for $k-1$. Let $v \in U_{1}$ be arbitrary. We wish to show that $k$-cliques containing $v$ belong to the same tight component in $K_{k}(G)$. For $2 \leqslant j \leqslant k$, let $U_{j}^{\prime}=U_{j} \cap N_{G}(v)$. Let $n^{\prime}=\min _{2 \leqslant j \leqslant k}\left|U_{j}^{\prime}\right|$, and note $n^{\prime}>(k-1) n / k$. Let $G^{\prime}$ be the subgraph of $G$ induced in $U_{2}^{\prime} \cup \cdots \cup U_{k}^{\prime}$, thus $G^{\prime}$ is a $(k-1)$-partite graph with clusters of size at least $n^{\prime}$ each. If $X_{1}, X_{2}$ are two $k$-cliques containing $v$, then $X_{1}^{\prime}=X_{1} \backslash\{v\}$, $X_{2}^{\prime}=X_{2} \backslash\{v\}$ correspond to two $(k-1)$-cliques in $G^{\prime}$. For $2 \leqslant j \leqslant k$, let $U_{j}^{\prime \prime} \subseteq U_{j}^{\prime}$ have size exactly $n^{\prime}$ such that $X_{1}^{\prime}, X_{2}^{\prime} \subseteq \bigcup_{2 \leqslant j \leqslant k} U_{j}^{\prime \prime}$, and set $\mathcal{U}^{\prime \prime}=\left\{U_{j}\right\}_{j=2}^{k}$. Let $G^{\prime \prime}$ be the subgraph of $G$ induced in $U_{2}^{\prime \prime} \cup \cdots \cup U_{k}^{\prime \prime}$. Note that $G^{\prime \prime}$ is a $\mathcal{U}^{\prime \prime}$-partite graph with

$$
\delta\left(G^{\prime \prime} ; \mathcal{U}^{\prime \prime}\right) \geqslant \delta(G ; \mathcal{U})-\left(n-n^{\prime}\right)>n^{\prime}+\frac{k-1}{k} n-n=n^{\prime}-\frac{n}{k}>\frac{k-2}{k-1} n^{\prime} .
$$

By the induction hypothesis, $K_{k-1}\left(G^{\prime \prime}\right)$ is tightly connected, and thus there exists a tight walk between $X_{1}^{\prime}$ and $X_{2}^{\prime}$ in $G^{\prime \prime} \subseteq G^{\prime}$. By Proposition 5.1, this implies all $k$-edges containing $v$ are tightly connected in $K_{k}(G)$.

Now let $v, v^{\prime}$ be two arbitrary vertices in $U_{1}$. By Proposition 5.2, to show that $K_{k}(G)$ is tightly connected, it is enough to prove that $L_{K_{k}(G)}(v)$ and $L_{K_{k}(G)}\left(v^{\prime}\right)$ share a $(k-1)$ edge. This is equivalent to showing that there exists a $(k-1)$-clique $X$ in $G$, which is in the common neighbourhood of $v$ and $v^{\prime}$ in $G$. Such a clique can be found greedily by applying Observation 5.27 repeatedly, first by finding $v_{2} \in U_{2}$ which is a common neighbour of $v, v^{\prime}$; then finding $v_{3} \in U_{3}$ common neighbour of $v, v^{\prime}, v_{2}$, and so on. This concludes the proof of the inductive step, and thus the proof whenever $r=k$, for all $k \geqslant 2$.

Next, we fix $k \geqslant 2$, and we show the $r \geqslant k$ case by induction in $r$. The base case $r=k$ is already done, so assume $r \geqslant k+1$. Let $G_{1}=G-U_{1}$ and $G_{2}=G-U_{r}$. By induction hypothesis, $K_{k}\left(G_{1}\right)$ and $K_{k}\left(G_{2}\right)$ are tightly connected. We finish by showing that there is a $(k-1)$-clique in $K_{k-1}(G)$ which is contained both in an edge of $K_{k}\left(G_{1}\right)$ and in an edge of $K_{k}\left(G_{2}\right)$. Greedily construct a $(k-1)$-clique $X$ in the clusters $U_{2}, \ldots, U_{k}$, applying Observation 5.27 iteratively as before. Applying Observation 5.27 again, we see $X$ can be extended to a clique in $K_{k}\left(G_{1}\right)$ by choosing an extra neighbour in $U_{1}$, and $X$ can be extended to a clique in $K_{k}\left(G_{2}\right)$ by choosing an extra neighbour in $U_{r}$.

The proof of Theorem 2.11 splits in two cases, depending if $r>k$ or not. For $\mathcal{H}=K_{k}(G)$, we show that $(G, \mathcal{H})$ is a robust zero-free Hamilton framework, when $r>k$. When $r=k$, we show that $(G, \mathcal{H})$ is a robust $\mathcal{U}$-partite Hamilton framework instead where $\mathcal{U}$ is the given partition of $V(G)$.

Proof of Theorem 2.11. Given $r, k, \Delta$, $\mu$, we fix $\mu^{\prime} \ll \mu, 1 / k$. Let $\mathcal{U}$ be a family of $r$ disjoint sets of size $n$ each. Let $G$ be a $\mathcal{U}$-partite graph with $\delta(G ; \mathcal{U})>\frac{k-1}{k} n$, and let $\mathcal{H}=K_{k}(G)$. 
By Proposition 3.6, each $v \in V(G)$ has at least $\mu^{\prime} n^{k}$ linked edges in $\mathcal{H}$. We separate the remaining analysis depending if $r=k$ or $r>k$.

Case 1: $r=k$. By Theorem 3.5 (Frameworks - bandwidth) and the above, it suffices to show that $(G, \mathcal{H})$ is a $\mu^{\prime}$-proto-robust $k$-partite Hamilton framework. Let $G^{\prime} \subseteq G$ be a U-partite $\mu^{\prime}$-approximation of $G$ and $\mathcal{H}^{\prime}=\mathcal{H} \cap K_{k}\left(G^{\prime}\right)$. We have to show that $\mathcal{H}^{\prime}$ is tightly connected and $\mathcal{H}^{\prime}$ has a perfect fractional matching.

Let $n^{\prime}=\left|U_{1} \cap V\left(G^{\prime}\right)\right|$. Since $G^{\prime}$ is a $\mathcal{U}$-partite $\mu^{\prime}$-approximation of $G$, we have $n^{\prime}=$ $\left|U_{j} \cap V\left(G^{\prime}\right)\right|$ for all $1 \leqslant j \leqslant k$. Moreover, for each $1 \leqslant j \leqslant k$, each $v \in U_{j}$, and any $\ell \neq j$, we have $\operatorname{deg}_{G^{\prime}}\left(v, U_{\ell}\right) \geqslant \operatorname{deg}_{G^{\prime}}\left(v, U_{\ell}\right)-\mu^{\prime} n \geqslant\left(\frac{k-1}{k}+\mu\right) n-\mu^{\prime} n>\frac{k-1}{k} n^{\prime}$. Therefore, $\delta\left(G^{\prime} ; \mathfrak{U}^{\prime}\right)>(k-1) n^{\prime} / k$ where $\mathfrak{U}^{\prime}$ is obtained from $\mathcal{U}$ by deleting the vertices not in $G^{\prime}$. Thus $K_{k}\left(G^{\prime}\right)$ is tightly connected by Lemma 5.28 , and since $n^{\prime}$ is large enough, $K_{k}\left(G^{\prime}\right)$ has a perfect matching by Theorem 2.10. This finishes the proof of the $r=k$ case.

Case 2: $r>k$. By Theorem 3.5 it suffices to show that $(G, \mathcal{H})$ is a $\mu^{\prime}$-proto-robust zerofree $k$-partite Hamilton framework. Let $G^{\prime} \subseteq G$ be a $\mu^{\prime}$-approximation of $G$, and let $\mathcal{H}^{\prime}=$ $\mathcal{H} \cap K_{k}\left(G^{\prime}\right)$. We need to show that $\mathcal{H}$ is tightly connected, has a perfect fractional matching and contains a $(k+1)$-clique.

Note however that $\left|U_{j} \cap V\left(G^{\prime}\right)\right|$ may differ for different choices of $1 \leqslant j \leqslant r$. Let $n^{\prime}=$ $\min _{1 \leqslant j \leqslant r}\left|U_{j} \cap V\left(G^{\prime}\right)\right|$ and note that $n^{\prime} \geqslant n-\mu^{\prime} k n$. For each $1 \leqslant j \leqslant r$, let $U_{j}^{\prime} \subseteq U_{j} \cap V\left(G^{\prime}\right)$ be an arbitrary set of size exactly $n^{\prime}$, and let $\mathcal{U}^{\prime}=\left\{U_{j}^{\prime}\right\}_{j=1}^{r}$. Let $G^{\prime \prime}$ be the subgraph of $G^{\prime}$ induced on the clusters of $\mathcal{U}^{\prime}$, and let $\mathcal{H}^{\prime \prime}=\mathcal{H}^{\prime} \cap K_{k}\left(G^{\prime}\right)$. Since $\mu^{\prime} \ll \mu$, we have $\delta\left(G^{\prime \prime} ; \mathcal{U}^{\prime}\right)>(k-1) n^{\prime} / k$. So by Observation 5.27 and Lemma 5.28 , it follows that $\mathcal{H}^{\prime \prime}$ is tightly connected and contains a $(k+1)$-clique. As $U_{1}^{\prime}, \ldots, U_{r}^{\prime}$ were chosen arbitrarily, the same follows for $\mathcal{H}^{\prime}$.

It still remains to show $\mathcal{H}$ has a perfect fractional matching. Let us first assume that the partition $\mathcal{U}^{\prime}=\left\{U_{j}^{\prime}\right\}_{j=1}^{r}$ is unbalanced. It follows that $f\left(\mathcal{U}^{\prime}\right):=r \max _{j}\left\{\left|U_{j}^{\prime}\right|\right\}-\sum_{j=1}^{r}\left|U_{j}^{\prime}\right|>0$. Suppose that, without loss of generality, $U_{k+1}^{\prime}$ has maximum size. By Lemma 5.27, there is a $(k+1)$-clique $X_{1}$ in $\mathcal{H}$ with one vertex in each of $U_{1}^{\prime}, \ldots, U_{k+1}^{\prime}$. Moreover, we can find, for $2 \leqslant j \leqslant r$, pairwise disjoint (and disjoint of $X_{1}$ ) edges $X_{j}$ in $\mathcal{H}$ such that $X_{j}$ has one vertex in $U_{j}^{\prime}, \ldots, U_{j+k-1}^{\prime}$ each, where the indices are computed modulo $r$. Let $X=\bigcup_{j=1}^{r} X_{j}$. It follows that $X$ has $k+1$ vertices in $X_{k+1}$ and $k$ vertices in all the other clusters. Moreover, it is easy to see that $X$ contains a perfect fractional matching. (Assign weight 1 to the edges and weight $1 / k$ to the edges in the $(k+1)$-clique.) Let $U_{j}^{\prime \prime}=U_{j}^{\prime} \backslash V(X)$ for $1 \leqslant j \leqslant r$, and let $\mathcal{U}^{\prime \prime}=\left\{U_{j}^{\prime \prime}\right\}_{j=1}^{r}$. It follows that $f\left(\mathcal{U}^{\prime \prime}\right)=f\left(\mathcal{U}^{\prime}\right)-1$. We continue this process until the remaining partition is balanced. Note that this is possible, since each cluster loses at most $(r+1) f\left(\mathcal{U}^{\prime}\right) \leqslant(r+1) \mu^{\prime} n \leqslant \mu n$ vertices in this process. Now the clusters are balanced, say of size $n^{\prime \prime}$ each. Let $p \leqslant n^{\prime \prime}$ be divisible by $k$ and as large as possible. Consider a subgraph obtained by choosing a $p$-set of vertices in each cluster. By Theorem 2.10, this subgraph contains a $K_{k}$-factor. There are $\left(\begin{array}{c}n^{\prime \prime} \\ p\end{array}\right)^{r}$ possible ways of selecting a subgraph of this type. Scale all the resulting matchings by $\left(\begin{array}{c}n^{\prime \prime} \\ p\end{array}\right)^{-r}$ and take their summation to obtain a perfect fractional matching of the remaining graph. Together with the other fractional matchings, this yields a perfect fractional matching of $\mathcal{H}$.

5.7. Robust expansion. In this section, we show that robust expansion together with a linear minimum degree is essentially equivalent to having a robust 2-uniform Hamilton framework. Recall the following result of Tutte [66, Corollary 30.1a].

Theorem 5.29 (Tutte's theorem). A graph $G$ has a perfect fractional matching if and only if $\left|\bigcup_{s \in S} N(s)\right| \geqslant|S|$ for every independent set $S$.

Given this, we can prove the following two simple propositions.

Proposition 5.30. Let $1 / n \ll \mu \ll \nu \leqslant \tau \ll \eta$. Let $G$ be a robust $(\nu, \tau)$-expander on $n$ vertices with $\delta(G) \geqslant \eta n$. Then $(G, G)$ is a $\mu$-robust aperiodic Hamilton framework. 
Proof. Let $G^{\prime}$ be a $(\mu, \mu)$-approximation of $G$. Since $\mu \ll \nu, \tau, \eta$, it follows that $G^{\prime}$ is a robust $(\nu / 2, \tau / 2)$-expander on $n$ vertices with $\delta(G) \geqslant(\eta / 2) n$. The robust expansion and minimumdegree conditions imply easily that $G^{\prime}$ is connected and not bipartite. The conditions also imply that $\left|\bigcup_{s \in S} N_{G^{\prime}}(s)\right| \geqslant|S|$ for every independent set $S$ in $G^{\prime}$. By Theorem $5.29, G^{\prime}$ has a perfect fractional matching.

Proposition 5.31. Let $1 / n \ll \nu \leqslant \tau \ll \eta \ll \mu$. Let $(G, G)$ be a $\mu$-robust aperiodic Hamilton framework on $n$ vertices. Then $G$ is a robust $(\nu, \tau)$-expander with $\delta(G) \geqslant \eta n$.

Proof. Since $(G, G)$ is a $\mu$-robust Hamilton framework, $G$ is $\mu n$-vertex-connected and in particular, $\delta(G) \geqslant \mu n \geqslant \eta n$. Now suppose, for sake of contradiction, that $G$ is not a robust $(\nu, \tau)$-expander. Then there is some set $S \subseteq V(G)$ with $\tau n \leqslant|S| \leqslant(1-\tau) n$ and $|R|<|S|+\nu n$ where $R=\mathrm{RN}_{\nu, G}(S)$. Let $R^{\prime} \subseteq R$ with $\left|R^{\prime}\right|<|S|$ be obtained from $R$ by deleting $\nu n$ vertices. Let $S^{\prime}=S \backslash\left(R \backslash R^{\prime}\right)$. We obtain $G^{\prime}$ from $G$ by deleting the vertices $R \backslash R^{\prime}$ and also all edges $x y$ with $x \in V(G) \backslash R$ and $y \in S \backslash R$. Since $1 / n \ll \nu \leqslant \tau \ll \eta \ll \mu$, $G^{\prime}$ is a $(\mu, \mu)$-approximation, and hence $\left(G^{\prime}, G^{\prime}\right)$ is a Hamilton framework. Note that $R^{\prime}$ contains $\bigcup_{s \in S^{\prime}} N_{G^{\prime}}(s)$ and $S^{\prime} \backslash R^{\prime}$ is an independent set in $G^{\prime}$. Moreover,

$$
\left|S^{\prime} \backslash R^{\prime}\right|=\left|S^{\prime}\right|-\left|R^{\prime} \cap S^{\prime}\right|>\left|R^{\prime}\right|-\left|R^{\prime} \cap S^{\prime}\right|=\left|\bigcup_{s \in S^{\prime} \backslash R^{\prime}} N_{G^{\prime}}(s)\right| .
$$

But this implies that $G^{\prime}$ does not have a perfect fractional matching, as mandated by Theorem 5.29. A contradiction.

Note that Proposition 5.30 together with Theorem 3.4 implies that robust expanders of sufficiently large minimum degree contain Hamilton cycles, and together with Theorem 3.5(ii) we recover Theorem 2.12. We remark that Theorem 3.5(iii) does not apply here, as there are triangle-free robust expanders.

\section{Regularity and the Blow-Up Lemma}

In the following, we introduce a few standard tools for embedding large sparse substructures in dense graphs.

6.1. Regular and super-regular pairs. Next, we collect a few concepts that are connected to graph regularity. Let $G$ be a graph and $A$ and $B$ be non-empty, disjoint subsets of $V(G)$. We write $e_{G}(A, B)$ for the number of edges in $G$ with one vertex in $A$ and one in $B$ and define the density of the pair $(A, B)$ to be $d_{G}(A, B)=e_{G}(A, B) /(|A||B|)$. The pair $(A, B)$ is $\varepsilon$-regular in $G$ if we have $\left|d_{G}\left(A^{\prime}, B^{\prime}\right)-d_{G}(A, B)\right| \leqslant \varepsilon$ for all $A^{\prime} \subseteq A$ with $\left|A^{\prime}\right| \geqslant \varepsilon|A|$ and $B^{\prime} \subseteq B$ with $\left|B^{\prime}\right| \geqslant \varepsilon|B|$. We say that $(A, B)$ is $(\varepsilon, d)$-regular if it is $\varepsilon$-regular and has density at least $d$. A vertex $b \in B$ has typical degree in an $(\varepsilon, d)$-regular pair $(A, B)$ if $\operatorname{deg}(b, A) \geqslant(d-\varepsilon)|A|$; and we say $b$ has atypical degree otherwise. We say a pair $(A, B)$ is $(\varepsilon, d)$-super-regular if it is $(\varepsilon, d)$-regular and contains no vertices of atypical degree (in $A$ or $B)$.

The next proposition by Böttcher, Schacht and Taraz [7, Proposition 8] states that regular and super-regular pairs are robust up to small alterations on their vertex sets. We denote the symmetric difference between sets $A$ and $B$ by $A \triangle B=(A \backslash B) \cup(B \backslash A)$.

Proposition 6.1 (Robust regular and super-regular pairs). Let $(A, B)$ be an $(\varepsilon, d)$-regular pair and let $\left(A^{\prime}, B^{\prime}\right)$ be a pair such that $\left|A \triangle A^{\prime}\right| \leqslant \alpha\left|A^{\prime}\right|$ and $\left|B \triangle B^{\prime}\right| \leqslant \beta\left|B^{\prime}\right|$ for some $0 \leqslant \alpha, \beta \leqslant 1$. Then $\left(A^{\prime}, B^{\prime}\right)$ is an $\left(\varepsilon^{\prime}, d^{\prime}\right)$-regular pair with

$$
\varepsilon^{\prime}=2 \varepsilon+6\left(\alpha^{1 / 2}+\beta^{1 / 2}\right) \quad \text { and } \quad d^{\prime}=d-2(\alpha+\beta) .
$$

If, moreover, $(A, B)$ is $(\varepsilon, d)$-super-regular and each vertex in $A^{\prime}$ has at least $\left(d-\varepsilon^{\prime}\right)\left|B^{\prime}\right|$ neighbours in $B^{\prime}$ and each vertex in $B^{\prime}$ has at least $\left(d-\varepsilon^{\prime}\right)\left|A^{\prime}\right|$ neighbours in $A^{\prime}$, then $\left(A^{\prime}, B^{\prime}\right)$ is $\left(\varepsilon^{\prime}, d^{\prime}\right)$-super-regular. 
6.2. Partitions, reduced graphs and blow-ups. Let $G, R$ be graphs and $\mathcal{V}=\left\{V_{i}\right\}_{i=1}^{t}$ be a partition of $V(G)$. We say that the partition $\mathcal{V}$ is $\kappa$-balanced (or just balanced when $\kappa=1$ ) if there exists $m \in \mathbb{N}$ such that we have $m \leqslant\left|V_{i}\right| \leqslant \kappa m$ for all $1 \leqslant i \leqslant t$. For a family $\mathcal{V}^{\prime}=\left\{V_{i}^{\prime}\right\}_{i=1}^{t}$ of subsets $V_{i}^{\prime} \subseteq V_{i}$, we denote by $G\left[\mathcal{V}^{\prime}\right]$ the subgraph of $G$ induced on $\bigcup_{i=1}^{t} V_{i}^{\prime}$. We say that $G$ is $\mathcal{V}$-partite if every edge has at most one vertex in the clusters of $\mathcal{V}$. We say $(G, \mathcal{V})$ is an $R$-partition if each part of $\mathcal{V}$ is non-empty, and whenever there are edges of $G$ between $V_{i}$ and $V_{j}$ the pair $i j$ is an edge of $R$ (in particular, there are no edges within clusters). We refer to $R$ as the reduced graph of $G$ or, more precisely, $(G, \mathcal{V})$.

Suppose now $(G, \mathcal{V})$ is an $R$-partition and $\mathcal{H}$ is a $k$-graph defined on $V(G)$. We define the $k$-graph induced by $(\mathcal{H}, \mathcal{V})$ to be the $k$-graph $\mathcal{J}$ on $t$ vertices, which contains an edge $K$ whenever $\mathcal{H}$ has an edge $e$ whose vertices are in the clusters of $K$. If $\mathcal{J}^{\prime}$ is a $k$-graph such that $\mathcal{J}^{\prime} \supseteq \mathcal{J}$, we say $(\mathcal{H}, \mathcal{V})$ is a $\mathcal{J}^{\prime}$-partition. Note that when $\mathcal{H} \subseteq K_{k}(G)$ (which will always be the case), then $\mathcal{J} \subseteq K_{k}(R)$.

For a $k$-graph $F$ on $t$ vertices, we define the $(F, \mathcal{V})$-blow-up $F^{*}$ to be the $k$-graph obtained by replacing each vertex $i$ with $V_{i}$ and each edge of $R$ with complete $k$-partite $k$-graphs. Note that, under this definition, $\left(F^{*}, \mathcal{V}\right)$ is an $F$-partition. When all clusters of $\mathcal{V}$ have common size $m$, we sometimes omit the reference to $\mathcal{V}$ and say that $F^{*}$ is the $(F, m)$-blow-up.

An $R$-partition $(G, \mathcal{V})$ is $(\varepsilon, d)$-regular if for each $i j \in E(R)$ the pair $\left(V_{i}, V_{j}\right)$ is $(\varepsilon, d)$ regular, that is, $d\left(V_{i}^{\prime}, V_{j}^{\prime}\right) \geqslant d-\varepsilon$ for all $V_{i}^{\prime} \subseteq V_{i}$ with $\left|V_{i}^{\prime}\right| \geqslant \varepsilon\left|V_{i}\right|$ and $V_{j}^{\prime} \subseteq V_{j}$ with $\left|V_{j}^{\prime}\right| \geqslant \varepsilon\left|V_{j}\right|$. Let $R^{\prime} \subseteq R$ be a spanning subgraph. We say that $(G, \mathcal{V})$ is $(\varepsilon, d)$-super-regular on $R^{\prime}$ if for each $i j \in E\left(R^{\prime}\right)$ the pair $\left(V_{i}, V_{j}\right)$ is $(\varepsilon, d)$-super-regular.

It is a well-known fact that every dense, regular pair contains a large super-regular subpair. The following proposition of Kühn, Osthus and Taraz [50, Proposition 8] generalises this insight to bounded-degree subgraphs of the reduced graph.

Proposition 6.2 (Super-regularising $\left.R^{\prime}\right)$. Let $1 / n \ll 1 / t \ll \varepsilon \ll d, 1 / \Delta_{R^{\prime}}$. Let $G$ be a graph on $n$ vertices and $\mathcal{V}=\left\{V_{i}\right\}_{i=1}^{t}$ be a balanced partition of $V(G)$. Let $R$ be a graph on $t$ vertices and $R^{\prime} \subseteq R$ be a spanning subgraph with $\Delta\left(R^{\prime}\right) \leqslant \Delta_{R^{\prime}}$. Suppose that $(G, \mathcal{V})$ is an $(\varepsilon, d)$-regular $R$-partition. Then there is a balanced family $\mathcal{V}^{\prime}=\left\{V_{i}^{\prime}\right\}_{i=1}^{t}$ of subsets $V_{i}^{\prime} \subseteq V_{i}$ of size at least $(1-\sqrt{\varepsilon}) n / t$ such that $\left(G\left[\mathcal{V}^{\prime}\right], \mathcal{V}^{\prime}\right)$ is an $(2 \varepsilon, d)$-regular $R$-partition, which is $(2 \varepsilon, d)$-super-regular on $R^{\prime}$.

We also require the following well-known facts, whose proofs we omit. The next proposition allows us to refine of regular partitions randomly while retaining their regularity properties.

Proposition 6.3 (Randomly refining regular pairs). Let $1 / n \ll 1 / t \ll \varepsilon \ll \varepsilon^{\prime} \ll d$ and $1 / n \ll 1 / q$. Suppose that $(G, \mathcal{V})$ is a $(1+\varepsilon)$-balanced $(\varepsilon, d)$-regular $R$-partition, where $G$ has $n$ vertices and $\mathcal{V}$ has $t$ clusters. Consider a refinement $\mathcal{V}^{*}$ of $\mathcal{V}=\left\{V_{i}\right\}_{i=1}^{t}$ obtained as follows. For every $1 \leqslant i \leqslant t$, we partition $V_{i}$ into $\left\{V_{i, j}\right\}_{j=1}^{q}$ by placing each vertex of $V_{i}$ uniformly at random in one of the parts $V_{i, 1}, \ldots, V_{i, q}$. Let $R^{*}$ be graph induced by $\left(R, \mathcal{V}^{*}\right)$. Then the probability that $\left(G, \mathcal{V}^{*}\right)$ is a $\left(1+\varepsilon^{\prime}\right)$-balanced $\left(\varepsilon^{\prime}, d\right)$-regular $R^{*}$-partition is at least $2 / 3$.

The following proposition tells us that most partite tuples of vertices located in pairwise regular clusters have a large common neighbourhood.

Proposition 6.4 (Joint degrees in regular pairs). Let $1 / m \ll 1 / t \ll \varepsilon \ll \varepsilon^{\prime} \ll d, 1 / k$. Let $G$ be a graph with a balanced vertex partition $\mathcal{V}=\left\{V_{i}\right\}_{i=1}^{k}$, which has parts of size $m$. Suppose that $(G, \mathcal{V})$ is an $(\varepsilon, d)$-regular $K_{k}$-partition. Then all but at most $\varepsilon^{\prime} m^{k}$ sets $S=\left\{v_{1}, \ldots, v_{k}\right\}$ with $v_{i} \in V_{i}$ have the property that $\left|\bigcap_{i \in S \backslash\{j\}} N\left(v_{i}\right) \cap V_{j}\right| \geqslant\left(d-\varepsilon^{\prime}\right)^{k-1} m$ for every $1 \leqslant j \leqslant k$.

6.3. The Regularity Lemma. Szemerédi's Regularity Lemma [70] allows us to partition the vertex set of a graph into clusters of vertices such that most pairs of clusters are regular. We will use the following degree form [45, Theorem 1.10] with pre-partition, which we restate here in terms of approximations and $R$-partitions. For families of sets $\mathcal{V}$ and $\mathcal{U}$, we say that $\mathcal{V}$ is $\mathcal{U}$-refining if every element of $\mathcal{V}$ is a subset of an element of $\mathcal{U}$.

Lemma 6.5 (Regularity Lemma). Let $1 / n \ll 1 / t_{1} \ll 1 / t_{0}, \varepsilon, d, 1 / r$ and $1 \leqslant q \leqslant k$. Let $G$ be a graph with an $(n, r)$-sized vertex partition $\mathcal{U}$. Then there are $t_{0} \leqslant t \leqslant t_{1}$ with $t \equiv q \bmod k$, 
a subgraph $G^{\prime} \subseteq G$ with a U-refining balanced partition $\mathcal{V}$ with tr clusters in total, $t$ clusters in each part of $\mathcal{U}$, and a graph $R$ such that

(S1) $G^{\prime}$ is a $\mathcal{U}$-partite $(\varepsilon, d+\varepsilon)$-approximation of $G$, and

(S2) $\left(G^{\prime}, \mathcal{V}\right)$ is an $(\varepsilon, d)$-regular $R$-partition.

6.4. The Blow-Up Lemma. In this subsection, we introduce the Blow-Up Lemma, which allows us to embed spanning subgraphs into regular pairs. The original Blow-Up Lemma was proved by Komlós, Sárközy and Szemerédi [42]. Here we use a variant of this result due to Allen, Böttcher, Hàn, Kohayakawa and Person [2, Lemma 1.21], which is particularly suitable for our approach. To give the statement of the lemma, we need a couple of definitions.

Let $G, H, R, R^{\prime}$ be graphs with $R^{\prime} \subseteq R$. Let $\mathcal{X}=\left\{X_{i}\right\}_{i=1}^{t}$ be a partition of $V(H)$ and $\mathcal{V}=\left\{V_{i}\right\}_{i=1}^{t}$ be a partition of $V(G)$, and let $\tilde{X}=\left\{\tilde{X}_{i}\right\}_{i=1}^{t}$ be a family of subsets of $V(H)$. The partitions $X$ and $\mathcal{V}$ are size-compatible if $\left|X_{i}\right|=\left|V_{i}\right|$ for all $1 \leqslant i \leqslant t$. The family $\tilde{X}=\left\{\tilde{X}_{i}\right\}_{i=1}^{t}$ is an $\left(\alpha, R^{\prime}\right)$-buffer for $(H, X)$ if for each $1 \leqslant i \leqslant t$, we have $\tilde{X}_{i} \subseteq X_{i}$ and $\left|\tilde{X}_{i}\right| \geqslant \alpha\left|X_{i}\right|$, and, for each $x \in \tilde{X}_{i}$ and $x y, y z \in E(H)$ with $y \in X_{j}$ and $z \in X_{k}$, we have $i j, j k \in E\left(R^{\prime}\right)$.

Finally, we introduce image restrictions, which will help us to pre-embed some vertices before applying the Blow-Up Lemma. Following the exposition of Allen et al. [2], the idea can be explained as follows. Suppose we wish to embed a graph $H^{+}$into a graph $G^{+}$, but unfortunately $G^{+}$does not meet the conditions of our Blow-Up Lemma. We find a subgraph $G$ of $G^{+}$which does meet the conditions of the Blow-Up Lemma, and we 'preembed' some vertices of $H^{+}$onto the vertices $V\left(G^{+}\right) \backslash V(G)$. This leaves the induced subgraph $H$ of $H^{+}$to embed into $G$. The image restrictions then originate from these preembedded vertices: if $x \in X_{i} \subseteq V(H)$ has neighbours $\left\{z_{1}, \ldots, z_{\ell}\right\}$ in $V\left(H^{+}\right) \backslash V(H)$ which are pre-embedded to $\left\{u_{1}, \ldots, u_{\ell}\right\}=J_{x} \subseteq V\left(G^{+}\right) \backslash V(G)$, then $J_{x}$ restricts the embedding of $x$ to $I_{x}=\bigcap_{u \in J_{x}} N_{G^{+}}(u) \cap V_{i}$. In the following definition, we do not explicitly refer to the graphs $H^{+}$and $G^{+}$, but only to abstract restricting sets $J_{x}$, so that we do not need to include the graphs $H^{+}$and $G^{+}$in the Blow-Up Lemma. In addition, to simplify notation, we define an image restriction set $I_{x}$ for each vertex $x$ of $H$. For most vertices $x$, however, this set will be the trivial set $I_{x}=X_{i}$ where $X_{i}$ is the part of $\mathcal{X}$ containing $x$.

Definition 6.6 (Image restrictions). Let $R$ be a graph on $t$ vertices, and $(H, X)$ be an $R$-partition and $(G, \mathcal{V})$ a size-compatible $(\varepsilon, d)$-regular $R$-partition, where $V(G) \subseteq V$.

Let $\mathcal{J}=\left\{I_{x}\right\}_{x \in V(H)}$ be a collection of subsets of $V(G)$, called image restrictions, and $\mathcal{J}=\left\{J_{x}\right\}_{x \in V(H)}$ be a collection of subsets of $V \backslash V(G)$, called restricting vertices. We say that $\mathcal{J}$ and $\mathcal{J}$ are a $\left(\varrho, \zeta, \Delta, \Delta_{J}\right)$-restriction pair if the following properties hold for each $1 \leqslant i \leqslant t$ and $x \in X_{i}$.

(I1) The set $X_{i}^{*} \subseteq X_{i}$ of image restricted vertices in $X_{i}$, that is, vertices such that $I_{x} \neq V_{i}$, has size $\left|X_{i}^{*}\right| \leqslant \varrho\left|X_{i}\right|$.

(I2) If $x \in X_{i}^{*}$, then $I_{x} \subseteq V_{i}$ is of size at least $\zeta d^{\left|J_{x}\right|}\left|V_{i}\right|$.

(I3) If $x \in X_{i}^{*}$, then $\left|J_{x}\right|+\operatorname{deg}_{H}(x) \leqslant \Delta$ and if $x \notin X_{i}^{*}$, then $J_{x}=\emptyset$.

(I4) Each vertex of $V$ appears in at most $\Delta_{J}$ of the sets of $\mathcal{J} .{ }^{4}$

With these definitions at hand, we can now state the full version of the Blow-Up Lemma that we will use.

Lemma 6.7 (Blow-Up Lemma). For all $\Delta, \Delta_{R^{\prime}}, \Delta_{J}, \kappa \geqslant 1$ and $\alpha, \zeta, d>0$ there exists $\varepsilon, \varrho>0$ such that for all $t_{1}$ there is an $n_{0}$ such that for all $n \geqslant n_{0}$ the following holds. Let $R$ be a graph on $t \leqslant t_{1}$ vertices and let $R^{\prime} \subseteq R$ be a spanning subgraph with $\Delta\left(R^{\prime}\right) \leqslant \Delta_{R^{\prime}}$. Let $H$ and $G$ be graphs on $n$ vertices with $\kappa$-balanced size-compatible vertex partitions $X=$ $\left\{X_{i}\right\}_{i=1}^{t}$ and $\mathcal{V}=\left\{V_{i}\right\}_{i=1}^{t}$, respectively. Let $\tilde{X}=\left\{\tilde{X}_{i}\right\}_{i=1}^{t}$ be a family of subsets of $V(H)$, let $\mathcal{J}=\left\{I_{x}\right\}_{x \in V(H)}$ be a family of image restrictions and $\mathcal{J}=\left\{J_{x}\right\}_{x \in V(H)}$ be a family of restricting vertices. Suppose that

\footnotetext{
${ }^{4}$ Note that this concept can be further simplified as in the work of Komlós, Sárközy and Szemerédi [42].
} 
(B1) $\Delta(H) \leqslant \Delta,(H, X)$ is an R-partition and $\tilde{X}$ is an $\left(\alpha, R^{\prime}\right)$-buffer for $(H, X)$,

(B2) $(G, \mathcal{V})$ is an $(\varepsilon, d)$-regular $R$-partition, which is $(\varepsilon, d)$-super-regular on $R^{\prime}$,

(B3) J and I form a $\left(\varrho, \zeta, \Delta, \Delta_{J}\right)$-restriction pair.

Then there is an embedding $\psi: V(H) \rightarrow V(G)$ such that $\psi(x) \in I_{x}$ for each $x \in V(H)$.

\section{INTERMEDIATE RESULTS}

This section contains a series of propositions that we require to prove Theorems 3.4 and 3.5.

7.1. Distributed matching. The following lemma shows that, in dense $k$-graphs, we can find a matching which intersects each subgraph of a given family of subgraphs simultaneously. Its proof is a standard application of concentration bounds.

Proposition 7.1 (Distributed matching). Let $1 / n \ll \eta \ll \pi \ll \gamma, 1 / k$. Let $\mathcal{H}$ be a $k$-graph on $n$ vertices. Let $\mathcal{F}$ be a collection of at most $2^{\eta n}$ subgraphs of $\mathcal{H}$, where each $\mathcal{F} \in \mathcal{F}$ has a least $\gamma n^{k}$ edges. Then there is a matching $\mathcal{M} \subseteq \mathcal{H}$ of size at most $\sqrt{\pi} n$ such that $\mathcal{M}$ has at least $\pi n$ edges of every subgraph $\mathcal{F} \in \mathcal{F}$.

7.2. Tight walks. The following facts will help us to navigate along tight components. Recall that a $k$-graph is tightly connected if there exists a closed tight walk which visits every edge. In what follows, we show we can control some properties of these walks.

Proposition 7.2. Let $\mathcal{J}$ be a tightly connected $k$-graph. Let $\left(w_{1}, \ldots, w_{k}\right)$ be an ordering of an edge in $\mathcal{J}$. Then there exists a closed walk $W$ which contains $\left(w_{1}, \ldots, w_{k}\right)$ as a subwalk, and visits every edge of $\mathcal{J}$.

Proof. Let $W$ be a closed walk which contains $\left(w_{1}, \ldots, w_{k}\right)$ and visits the maximum number of edges of $\mathcal{J}$. If $W$ does not visit every edge of $\mathcal{J}$, there must exist edges $X, Y$ in $\mathcal{J}$ such that $|X \cap Y|=k-1$, and $W$ visits $X$ but not $Y$. Similarly to the proof of Proposition 5.1, we can extend $W$ to obtain a walk $W^{\prime}$ which also contains $\left(w_{1}, \ldots, w_{k}\right)$ as a subwalk and visits every edge visited by $W$ and also $Y$, contradicting the maximality.

Next, we bound the length of a walk visiting all the edges of a tightly connected graph.

Proposition 7.3 (Closed tight walk visiting all edges). Let $\mathcal{J}$ be a tightly connected $k$-graph on $t \geqslant k$ vertices and $0 \leqslant q \leqslant k-1$. Then there exists a closed tight walk $W$ which visits all edges of $\mathcal{J}$ and has length at most $k^{2} t^{k}+k\left(\begin{array}{l}t \\ k\end{array}\right) t^{k}$. Moreover, if $\mathcal{J}$ contains a closed walk of length coprime to $k$, then we can also assume $W$ has length congruent to $q \bmod k$.

Proof. Suppose first that $\mathcal{J}$ contains a walk of length coprime to $k$, we describe the complementary case at the end. Let $C=\left(w_{1}, \ldots, w_{\ell}\right)$ be a walk in $\mathcal{J}$ of length coprime to $k$, and suppose $C$ has minimum length among all the closed walks with this property. We claim $\ell \leqslant k t^{k}$. Indeed, assume otherwise. Since there are at most $t^{k}$ sequences of $k$ distinct vertices of $V(\mathcal{J})$, there must exist some sequence which has at least $k$ appearances in $C$. We can write $C=C_{1} \cdots C_{k}$ as concatenation of walks $C_{1}, \ldots, C_{k}$ such that each $C_{i}$ begins with the same sequence of $k$ vertices. A pigeonhole argument shows the existence of $1 \leqslant i \leqslant j \leqslant k$ with $(i, j) \neq(1, k)$ such that the walk $C_{i} \cdots C_{j}$ has length divisible by $k$. But then $C_{1} \cdots C_{i-1} C_{j+1} \cdots C_{k}$ is a closed walk of length congruent to $\ell$ mod $k$ and shorter than $C$, a contradiction.

Let $\left(x_{1}, \ldots, x_{k}\right)$ be a subwalk of $C$. By Proposition 7.2, there exists a walk $W$ which contains all edges of $\mathcal{J}$ and contains $\left(x_{1}, \ldots, x_{k}\right)$ as a subwalk. Let $W$ be a walk of minimum length with those two properties. Let $e_{1}, \ldots, e_{r}$ be an enumeration of the edges of $\mathcal{J}$ according to the first time they are visited on $W$, in particular we can assume $e_{1}=\left\{x_{1}, \ldots, x_{k}\right\}$. Let $W_{i}$ be the subwalk of $W$ between the first appearance of $e_{i}$ and the first appearance of $e_{i+1}$, in cyclic order, so $W=W_{1} W_{2} \cdots W_{r}$. By minimality, each $W_{i}$ cannot contain two distinct subwalks of length $k$ which correspond to exactly the same ordered $k$-tuple of vertices, otherwise we could replace $W_{i}$ in $W$ by a shortened $W_{i}^{\prime}$ and keep all of the desired properties. There are at most $t(t-1) \cdots(t-k+1)$ ordered $k$-tuples of distinct vertices of 
$\mathcal{J}$, so each $W_{i}$ has length at most $t(t-1) \cdots(t-k+1)+k-1 \leqslant t^{k}$. Since there are at most $\left(\begin{array}{l}t \\ k\end{array}\right)$ edges in $\mathcal{J}$, we obtain that $W$ has length at most $\left(\begin{array}{l}t \\ k\end{array}\right) t^{k}$.

We obtain the desired walk as follows: concatenate at most $k$ copies of $W$ with itself, to obtain a walk $W^{\prime}$ of length divisible by $k$ and at most $k\left(\begin{array}{l}t \\ k\end{array}\right) t^{k}$, which visits all edges of $\mathcal{J}$. Since the length $\ell$ of $C$ is coprime with $k$, there exists $0 \leqslant a \leqslant k-1$ such that $a \ell \equiv q \bmod k$. Concatenate $a$ copies of $C$ with itself, to obtain a walk $C^{\prime}$ of length congruent to $q \bmod k$ and at most $k^{2} t^{k}$. Then merge $C^{\prime}$ and $W^{\prime}$ into a single walk: this is possible since both share the subwalk $\left(x_{1}, \ldots, x_{k}\right)$. The final walk has length congruent to $q \bmod k$ and at most $k^{2} t^{k}+k\left(\begin{array}{l}t \\ k\end{array}\right) t^{k}$, as required. Finally, if $\mathcal{J}$ does not contain a walk of length coprime to $k$, the task becomes simpler: build $W$ as before, now starting from an arbitrary $\left(x_{1}, \ldots, x_{k}\right)$.

Finally, the next proposition allows us to find walks of controlled length between some prescribed ordered tuples.

Proposition 7.4. Let $1 / t \ll 1 / k$. Let $\mathcal{J}$ be a tightly connected $k$-graph on $t$ vertices and let $K$ be a set of $k+1$ vertices of $\mathcal{J}$ spanning a clique. For any ordered edges $e_{1}=\left(v_{1}, \ldots, v_{k}\right) \in$ $V(\mathcal{J})^{k}$ and $e_{2}=\left(c_{1}, \ldots, c_{k}\right) \in K^{k}$, there exists a walk beginning with $e_{1}$ and ending with $e_{2}$ of length exactly $k t^{k}$.

Proof. Since $\mathcal{J}$ is tightly connected, there exists a tight walk starting from $e_{1}$ and whose last $k$ vertices are those of $e_{2}$ (possibly in a different order). Let $W_{1}$ be such a walk of minimum length. The length of $W_{1}$ must be at most $t^{k}$. Let $f_{2}$ be the last $k$ ordered vertices of $W_{1}$.

Now we show that there is a short walk from $f_{2}$ to $e_{2}$ using vertices from $K$ only. Suppose $K=\left\{c_{1}, \ldots, c_{k+1}\right\}$ and $f_{2}=\left(c_{1}, \ldots, c_{k}\right)$. Then, given $1 \leqslant i<j \leqslant k$, the sequence

$$
c_{1} \cdots c_{k} c_{1} \cdots c_{i-1} c_{k+1} c_{i+1} \cdots c_{j-1} c_{i} c_{j+1} \cdots c_{k} c_{1} \cdots c_{i-1} c_{j} c_{i+1} \cdots c_{j-1} c_{i} c_{j+1} \cdots c_{k}
$$

is a walk in $\mathcal{J}[K]$ of length $3 k$, which starts with $f_{2}$ and ends with $f_{2}^{\prime}$, consisting of the same ordered vertices of $f_{2}$ but now with $c_{i}$ and $c_{j}$ swapped. By repeating this, we can swap two more vertices, and iterate. If we need $s$ swaps to rearrange $f_{2}$ to get $e_{2}$, then there is a walk $W_{2}$ of length $k+s 2 k$ which starts with $f_{2}$ and ends with $e_{2}$. Since we can always reach $e_{2}$ with at most $k$ swaps, we can assume $W_{2}$ has length at most $k+2 k^{2}$. Joining $W_{1}$ and $W_{2}$ together we get a walk $W_{3}$ of length at most $t^{k}+2 k^{2}$ which starts with $e_{1}$ and ends with $e_{2}$. By concatenating $W_{3}$ with at most $k$ copies of the walk $c_{k+1} e_{2}$, we get a walk $W_{4}$ of length at most $t^{k}+2 k^{2}+k(k+1)$ which starts with $e_{1}$, ends with $e_{2}$, and has length 0 modulo $k$. Since $1 / t \ll 1 / k, 2 k^{2}+k(k+1) \leqslant(k-1) t^{k}$. We can concatenate $W_{4}$ with copies of $e_{2}$ to obtain a walk $W$ which starts with $e_{1}$, ends with $e_{2}$, and has length exactly $k t^{k}$, as desired.

7.3. Allocations. In this section, we present some results that will help us to (re)allocate vertices in Lemma 8.3 (Lemma for $C$ ). Let $\mathcal{H}$ be a $k$-graph. We define the 2 -graph $\hat{\mathcal{H}}$ on $V(\mathcal{H})$ by joining two vertices $x$ and $y$ if their link graphs have a common edge. For $U \subseteq V(\mathcal{H})$, we denote the graph induced by $U$ in $\hat{\mathcal{H}}$ by $\hat{\mathcal{H}}[U]$. A simple induction shows that if there is a tight walk in $\mathcal{H}$ of length congruent to $1 \bmod k$ that starts with $x$ and ends with $y$, then $x$ and $y$ are in the same component in $\hat{\mathcal{H}}$. We say that a vertex in a hypergraph is isolated if it is not contained in any edge.

Proposition 7.5. Let $k \geqslant 2$ and $r \in\{1, k\}$, and let $\mathcal{U}$ be an $(n, r)$-sized partition. Let $\mathcal{H}$ be a U-partite $k$-graph, which has no isolated vertices and is tightly connected. If $r=1$, suppose further that $\mathcal{H}$ contains a closed tight walk of length coprime to $k$. Then $\hat{\mathcal{H}}[U]$ is connected for every $U \in \mathcal{U}$.

Proof. Consider vertices $x, y \in U$ for some fixed $U \in \mathcal{U}$. By the previous observations, it suffices to find a tight walk in $\mathcal{H}$ which begins with $x$, ends with $y$, and has length congruent to 1 modulo $k$. Since $\mathcal{H}$ has no isolated vertices, there are edges $e$ and $f$ with $x \in e$ and $y \in f$.

Consider the case $r=k$ first. Since $\mathcal{H}$ is tightly connected, there exists a closed tight walk $W$ visiting every edge of $\mathcal{H}$, in particular $e$ and $f$. We deduce there exists a subwalk 
$W^{\prime} \subseteq W$ which starts with $x$ and ends with $y$. In particular, when $r=k, \mathcal{H}$ is $k$-partite, thus $W^{\prime}$ must necessarily have length $1 \bmod k$, as required.

For the case $r=1$, we also suppose $\mathcal{H}$ contains a closed tight walk of length coprime to $k$. Hence, by Proposition 7.3 there is a closed tight walk $W$ which visits every edge and has length 1 modulo $k$. Without loss of generality, we can suppose $W$ starts with $x$. Let $W^{\prime}$ be the subwalk of $W$ from its start $x$ until the first appearance of $y$, and let $\ell$ be its length. Let $0 \leqslant a<k$ be such that $a+\ell \equiv 1 \bmod k$. Construct $W^{\prime \prime}$ by concatenating $a$ copies of $W$ and one copy of $W^{\prime}$. Then $W^{\prime \prime}$ is a tight walk in $\mathcal{H}$ which begins with $x$, ends with $y$, and has length congruent to 1 modulo $k$, as required.

The following proposition shows that, under suitable conditions, for any bounded integer allocation $\mathbf{b}: V(\mathcal{H}) \rightarrow \mathbb{Z}$ over the vertices of a hypergraph there exists some appropriately bounded allocation $\mathbf{w}: E(\mathcal{H}) \rightarrow \mathbb{Z}$, where every vertex receives the exact amount $\mathbf{b}(u)$ from its incident edges. The lemma and its proof have been taken from our earlier work with Garbe, Lo and Mycroft [27], and can be found in Appendix C.1. Recall that $\|\mathbf{v}\|_{\infty}=$ $\max _{x \in X}|\mathbf{v}(x)|$ for a set $X$ and a vector $\mathbf{v} \in \mathbb{R}^{X}$.

Proposition 7.6. Let $\mathcal{U}$ be an $(n, r)$-sized partition, and let $\mathcal{H}$ be a $\mathcal{U}$-partite k-graph. Suppose that $\hat{\mathcal{H}}[U]$ is connected for each $U \in \mathcal{U}$. Let $\mathbf{b} \in \mathbb{Z}^{V(\mathcal{H})}$ such that $\sum_{v \in U} \mathbf{b}(v)=0$ for every $U \in \mathcal{U}$ and $\|\mathbf{b}\|_{\infty} \leqslant s$. Then there exists $\mathbf{w} \in \mathbb{Z}^{E(\mathcal{H})}$ with $\mathbf{b}(v)=\sum_{v \ni e} \mathbf{w}(e)$ for all $v \in V(\mathcal{H})$ and $\|\mathbf{w}\|_{\infty} \leqslant k s n^{2}$.

7.4. Bounded degree cover. The next lemma allows us to find an edge cover of bounded degree in a graph with a perfect fractional matching. This will be useful when finding the $R^{\prime} \subseteq R$ in the setting of the Blow-Up Lemma. The idea and proof comes again from our earlier work with Garbe, Lo and Mycroft [27] and can be found in Appendix C.2.

Lemma 7.7 (Bounded degree cover). Let $\mathcal{J}$ be a k-graph on $n$ vertices which admits a perfect fractional matching. Then there is a spanning subgraph $\mathcal{J}^{\prime} \subseteq \mathcal{J}$ with $\Delta\left(\mathcal{J}^{\prime}\right) \leqslant k^{2}+1$.

7.5. Frameworks and regularity. In this section, we develop a few tools that help us to combine frameworks with regular partitions. To begin, we show that the reduced graph inherits the property of being a Hamilton framework. Consider families of disjoint sets $\mathcal{U}$ and $\mathcal{V}=\left\{V_{i}\right\}_{i=1}^{t}$, where $\mathcal{V}$ is $\mathcal{U}$-refining. We say that a partition $\mathcal{W}$ of $\{1, \ldots, t\}$ is induced by $(\mathcal{V}, \mathcal{U})$, if each part of $\mathcal{W}$ contains precisely the indices of the clusters of $\mathcal{V}$ which are contained in some part of $\mathcal{U}$.

Proposition 7.8. Let $1 / n \ll 1 / t \ll \mu, 1 / k$, and $r \in\{1, k\}$. Let $\mathcal{U}$ be an $(n, r)$-sized partition, and let $(G, \mathcal{H})$ be a $(2 \mu)$-robust $\mathcal{U}$-partite $k$-uniform (aperiodic/zero-free) Hamilton framework. Let $(G, \mathcal{V})$ be a balanced $\mathcal{U}$-refining $R$-partition with $t$ clusters in each part of $\mathcal{U}$. Let $\mathcal{J}$ be the $k$-graph induced by $(\mathcal{H}, \mathcal{V})$. Let $\mathcal{W}$ be the $(t, r)$-sized partition of $R$ induced by $(\mathcal{V}, \mathcal{U})$. Then $(R, \mathcal{J})$ is a $\mu$-robust $\mathcal{W}$-partite (aperiodic/zero-free) Hamilton framework.

Proof. We first show part (R1) of Definition 3.3 (Robustness). Let $R^{\prime} \subseteq R$ be an arbitrary $\mathcal{W}$-partite $\mu$-approximation of $R$. Let $\mathcal{V}^{\prime}$ be obtained from $\mathcal{V}$ by deleting the clusters whose indices are not in $V\left(R^{\prime}\right)$. We obtain $G^{\prime} \subseteq G$ by deleting all vertices of clusters $V_{i}$ with $i \notin V\left(R^{\prime}\right)$ and all edges between $V_{i}$ and $V_{j}$ whenever $i j \notin E\left(R^{\prime}\right)$. Let $m$ denote the common cluster size of $\mathcal{V}$. Since $n=m t$ and $1 / n \ll 1 / t \ll \mu$, it follows that $G^{\prime}$ is a $\mathcal{U}$-partite $(2 \mu)$ approximation of $G$. Let $\mathcal{H}^{\prime}=\mathcal{H} \cap K_{k}\left(G^{\prime}\right)$. By Definition 3.1 (Hamilton framework) and the assumption, $\left(G^{\prime}, \mathcal{H}^{\prime}\right)$ is a Hamilton framework, which is aperiodic or zero-free if $(G, \mathcal{H})$ was also aperiodic or zero-free.

We have to show that $\left(R^{\prime}, \mathcal{J}^{\prime}\right)$ is a Hamilton framework for $\mathcal{J}^{\prime}=\mathcal{J} \cap K_{k}\left(R^{\prime}\right)$. Observe that connectivity, aperiodicity and zero-freeness (as detailed in Definition 3.1) follow immediately from the respective property of $\left(G^{\prime}, \mathcal{H}^{\prime}\right)$ and the definition of $R$-partitions. For the matchability, consider a perfect fractional matching $\mathbf{w}: E\left(\mathcal{H}^{\prime}\right) \rightarrow \mathbb{R}$. We then construct a perfect fractional matching $\mathbf{w}^{\prime} \in \mathbb{R}^{E\left(\mathcal{J}^{\prime}\right)}$ of $\mathcal{J}^{\prime}$ by setting $\mathbf{w}^{\prime}(K)$ to be the sum of the weights $\mathbf{w}(e) / m$ over all edges $e$ that have their vertices in the $k$ clusters of $K$. 
Finally, part (R2) of Definition 3.3 follows by a simple averaging argument, since $1 / n \ll$ $1 / t \ll \mu, 1 / k$.

Reduced graphs of Hamilton frameworks also inherit a more nuanced matching property, which plays an important role in the process of allocating vertices in Lemmas 8.3 and 9.2. Recall the definition of blow-ups from Section 6.2. Informally, cluster-matchable graphs are such that their blow-ups by slightly imbalanced clusters admit large matchings.

Definition 7.9 (Cluster-matchable). Let $\mathcal{J}$ be a $\mathcal{W}$-partite graph where $\mathcal{W}$ is a $(t, r)$-sized partition of $\mathcal{J}$. We say that $\mathcal{J}$ is $(\alpha, \beta, \mathcal{W})$-cluster-matchable if the following holds. Let $\mathcal{X}$ be any $(1+\alpha)$-balanced family of disjoint sets whose cluster indices are the vertices of $\mathcal{J}$. Suppose furthermore that, for every $W \in \mathcal{W}$, the total number of elements in clusters of $\mathcal{X}$ with index in $W$ is the same. Then the $(\mathcal{J}, \mathcal{X})$-blow-up $\mathcal{J}^{*}$ has a matching missing at most $\beta v\left(\mathcal{J}^{*}\right)+t^{k-1}$ vertices in each cluster of $\mathcal{X}$.

Proposition 7.10. Let $0<\mu \leqslant 1, k \geqslant 2$, and $r \in\{1, k\}$ such that $\mu^{2} n \geqslant 4 t$. Let $\mathcal{U}$ be an $(n, r)$-sized partition, Let $G$ be a U-partite graph, and let $R$ be a graph with a $(t, r)$-sized partition $\mathcal{W}$. Let $(G, \mathcal{V})$ be a $\mathcal{U}$-refining balanced $R$-partition. Suppose that $(G, \mathcal{H})$ is a $\mu$ robust $\mathcal{U}$-partite $k$-uniform Hamilton framework, and let $\mathcal{J}$ be the $k$-graph induced by $(\mathcal{H}, \mathcal{V})$. Then $\mathcal{J}$ is $(\mu, 2 / n, \mathcal{W})$-cluster-matchable.

Proof. Let $\mathcal{J}^{*}$ be a $(\mathcal{J}, X)$-blow-up for some $(1+\mu)$-balanced family of disjoint sets $\mathcal{X}=$ $\left\{X_{i}\right\}_{i=1}^{r t}$ such that, for every $W \in \mathcal{W}$, the number of elements in clusters of $\mathcal{X}$ with index in $W$ is the same. We have to show that $\mathcal{J}^{*}$ contains a matching missing at most $2 v\left(\mathcal{J}^{*}\right) / n+t^{k-1}$ vertices in each cluster of $X$.

By assumption, there exists $m^{\prime \prime}$ such that $m^{\prime \prime} \leqslant\left|X_{i}\right| \leqslant(1+\mu) m^{\prime \prime}$ for all $1 \leqslant i \leqslant r t$. This implies there is an $m^{\prime}$ such that $m^{\prime} /(1+\mu) \leqslant\left|X_{i}\right| \leqslant m^{\prime}$ for each $1 \leqslant i \leqslant r t$. Let $m$ be the common cluster size of $\mathcal{V}=\left\{V_{i}\right\}_{i=1}^{r t}$. Choose subsets $V_{i}^{\prime} \subseteq V_{i}$ of size $\left\lfloor\left|X_{i}\right| m / m^{\prime}\right\rfloor$. Let $\mathcal{V}^{\prime}=\left\{V_{i}^{\prime}\right\}_{i=1}^{r t}, G^{\prime}=G\left[\mathcal{V}^{\prime}\right]$, and $\mathcal{H}=\mathcal{H}^{\prime} \cap K_{k}\left(G^{\prime}\right)$. Recall that by assumption the number of elements in clusters of $\mathcal{X}$ is the same over each part of $\mathcal{W}$. Hence, after accounting for rounding errors, we have $\left|U \cap V\left(G^{\prime}\right)\right|=\left|U^{\prime} \cap V\left(G^{\prime}\right)\right| \pm t$ for all $U, U^{\prime} \in \mathcal{U}$. To make this an equality, we delete up to $t$ vertices of every part of $\mathcal{U}$, keeping the names for convenience. Consider a part $U \in \mathcal{U}$ and suppose that without loss of generality $U$ contains clusters $V_{1}, \ldots, V_{t}$ of $\mathcal{V}$. We have

$$
\left|U \cap V\left(G^{\prime}\right)\right| \geqslant\left(\sum_{i=1}^{t}\left\lfloor\left|X_{i}\right| m / m^{\prime}\right\rfloor\right)-t \geqslant\left(\sum_{i=1}^{t}\lfloor m /(1+\mu)\rfloor\right)-t \geqslant(1-\mu)|U|,
$$

where the last step uses $m=n / t \geqslant 4 \mu^{-2}$ and $|U|=n$. It thus follows that $G^{\prime}$ is a $\mathcal{U}$ partite $\mu$-approximation of $G$. Since $(G, \mathcal{H})$ is a $\mu$-robust $\mathcal{U}$-partite Hamilton framework, we conclude that $\left(G^{\prime}, \mathcal{H}^{\prime}\right)$ is a Hamilton framework. Hence there is a perfect fractional matching $\mathbf{w}: E\left(\mathcal{H}^{\prime}\right) \rightarrow \mathbb{R}$.

We define $\mathbf{w}^{\prime}: E(R) \rightarrow \mathbb{R}$ by setting $\mathbf{w}^{\prime}(K)=\left\lfloor\frac{m^{\prime}}{m} \sum_{e \in \mathcal{H}_{K}} \mathbf{w}(e)\right\rfloor$ for every edge $K$ in $R$ where $\mathcal{H}_{K} \subseteq E\left(\mathcal{H}^{\prime}\right)$ denotes the set of all $k$-edges $e \in E\left(\mathcal{H}^{\prime}\right)$ which have one vertex in each of the clusters of $K$. Observe that, for every $1 \leqslant i \leqslant r t$,

$$
\sum_{K \ni i} \sum_{e \in \mathcal{H}_{k}} \mathbf{w}(e)=\left|V_{i}^{\prime}\right|=\left\lfloor\left|X_{i}\right| \frac{m}{m^{\prime}}\right\rfloor,
$$

since $\mathbf{w}$ is a perfect fractional matching. In particular, by equation (7.1) we get

$$
\sum_{K \ni i} \mathbf{w}^{\prime}(K)=\sum_{K \ni i}\left\lfloor\frac{m^{\prime}}{m} \sum_{e \in \mathcal{H}_{K}} \mathbf{w}(e)\right\rfloor \leqslant \frac{m^{\prime}}{m} \sum_{K \ni i} \sum_{e \in \mathcal{H}_{K}} \mathbf{w}(e)=\frac{m^{\prime}}{m}\left\lfloor\left|X_{i}\right| \frac{m}{m^{\prime}}\right\rfloor \leqslant\left|X_{i}\right|
$$

for every $1 \leqslant i \leqslant r t$. By the definition of $\mathcal{J}^{*}$ and $\sum_{K \ni i} \mathbf{w}^{\prime}(K) \leqslant\left|X_{i}\right|$, we may greedily select a matching $M \subseteq \mathcal{J}^{*}$ which contains exactly $\mathbf{w}^{\prime}(K)$ many $K$-edges for each $K \in E(R)$. 
Using equation (7.1) again, it follows that $M$ covers at least

$$
\begin{aligned}
\sum_{K \ni i} \mathbf{w}^{\prime}(K) & =\sum_{K \ni i}\left\lfloor\frac{m^{\prime}}{m} \sum_{e \in \mathcal{H}_{K}} \mathbf{w}(e)\right\rfloor \geqslant \sum_{K \ni i}\left(\frac{m^{\prime}}{m} \sum_{e \in \mathcal{H}_{K}} \mathbf{w}(e)-1\right) \geqslant \frac{m^{\prime}}{m}\left\lfloor\left|X_{i}\right| \frac{m}{m^{\prime}}\right\rfloor-t^{k-1} \\
& \geqslant \frac{m^{\prime}}{m}\left(\left|X_{i}\right| \frac{m}{m^{\prime}}-1\right)-t^{k-1}=\left|X_{i}\right|-\frac{m^{\prime}}{m}-t^{k-1} \geqslant\left|X_{i}\right|-\frac{2 v\left(\mathcal{J}^{*}\right)}{n}-t^{k-1}
\end{aligned}
$$

vertices of each cluster $X_{i}$, where in the last inequality we used that $m=n / t$ and $m^{\prime} \leqslant$ $(1+\mu) v\left(\mathcal{J}^{*}\right) /(r t) \leqslant 2 v\left(\mathcal{J}^{*}\right) / t$ to get $m^{\prime} / m \leqslant 2 v\left(\mathcal{J}^{*}\right) / n$.

The next proposition states that a robust Hamilton framework $(G, \mathcal{H})$ admits a 'reduced framework' $(R, \mathcal{J})$, which approximately inherits its properties. Alone, this is a consequence of the Regularity Lemma (Lemma 6.5) and Proposition 7.8. But additionally, we will also ensure the existence of a small family $\mathcal{F}$ of subgraphs of $\mathcal{J}$, which allows us to (re)integrate any small number of vertices of $G$ into $\mathcal{V}$. We require this property to deal with these exceptional vertices in the proofs of the Lemmas for $G$ (Lemmas 8.2 and 9.1). We remark that a similar argument was used by Ebsen, Maesaka, Reiher, Schacht and Schülke [22].

Lemma 7.11 (Regular partition with entry points). Let $1 / n \ll 1 / t_{1} \ll 1 / t_{0} \ll \varepsilon \ll d \ll$ $\eta \ll \gamma \ll \mu, 1 / k$ and $1 \leqslant q \leqslant k$ and $r \in\{1, k\}$. Let $\mathcal{U}$ be an $(n, r)$-sized partition, and let $(G, \mathcal{H})$ be a $\mu$-robust $\mathcal{U}$-partite $k$-uniform Hamilton framework.

Then there are $t_{0} \leqslant t \leqslant t_{1}$ with $t \equiv q \bmod k$, a subgraph $G^{\prime} \subseteq G$ with a balanced U-refining partition $\mathcal{V}$ with $t$ clusters in each part of $\mathcal{U}$, and a graph $R$ with a $(t, r)$-sized partition $\mathcal{W}$ induced by $(\mathcal{V}, \mathcal{U})$, which satisfy that

(R1) $G^{\prime}$ is a U-partite $(\varepsilon, 4 d)$-approximation of $G$ and

(R2) $\left(G^{\prime}, \mathcal{V}\right)$ is an $(\varepsilon, d)$-regular $R$-partition.

Let $\mathcal{H}^{\prime}=\mathcal{H} \cap K_{k}\left(G^{\prime}\right)$, and let $\mathcal{J}$ be the $k$-graph induced by $\left(\mathcal{H}^{\prime}, \mathcal{V}\right)$. Then

$(\mathrm{R} 3)(R, \mathcal{J})$ is a $(\mu / 4)$-robust $\mathcal{W}$-partite Hamilton framework and $(\mu / 4,2 / n, \mathcal{W})$-clustermatchable, and

(R4) there is a collection $\mathcal{F}$ of subgraphs of $\mathcal{J}$ such that

(i) $\mathcal{F}$ has at most $2^{\eta t}$ elements,

(ii) each subgraph in $\mathcal{F}$ has at least $\gamma(r t)^{k}$ edges,

(iii) for every vertex $v \in V(G)$, there is an $\mathcal{F} \in \mathcal{F}$ such that $v$ has at least $\gamma(n /(r t))^{k}$ linked edges in $\mathcal{H}^{\prime}$ in the clusters of $\mathcal{V}$ corresponding to every $K \in E(\mathcal{F})$.

Finally, if $(G, \mathcal{H})$ is $\mu$-robust aperiodic (resp. zero-free), then $(R, \mathcal{J})$ is $(\mu / 4)$-robust aperiodic (resp. zero-free) as well.

Proof. We introduce new constants $t^{*} \in \mathbb{N}$ and $\varepsilon^{\prime}>0$ such that

$$
1 / n \ll 1 / t_{1}^{*} \ll 1 / t_{1} \ll 1 / t_{0} \ll \varepsilon \ll \varepsilon^{\prime} \ll d \ll \eta \ll \gamma \ll \mu, 1 / k .
$$

In particular, we choose $t_{1}$ so that Lemma 6.5 (Regularity Lemma) holds with parameters $t_{0}, d, \varepsilon, k$. We further choose these constants such that the various inequalities along the proof are satisfied. Finally, let $1 \leqslant q \leqslant k$. We start with the case where $(G, \mathcal{H})$ is a $\mu$-robust $\mathcal{U}$-partite $k$-uniform Hamilton framework. (The cases where $(G, \mathcal{H})$ is aperiodic or zero-free will be discussed in the end.)

Step 1: Obtaining a regular partition. We apply Lemma 6.5 to $G$ with input $\varepsilon, d, t_{0}, k, q$ to obtain $t_{0} \leqslant t \leqslant t_{1}$ with $t \equiv q \bmod k$, a subgraph $G^{\prime} \subseteq G$ with a U-refining balanced partition $\mathcal{V}=\left\{V_{i}\right\}_{i=1}^{r t}$ of $V\left(G^{\prime}\right)$ with $t$ clusters in every part of $\mathcal{U}$ and a graph $R$ such that

(S1) $G^{\prime}$ is a $\mathcal{U}$-partite $(\varepsilon, d+\varepsilon)$-approximation of $G$ and

(S2) $\left(G^{\prime}, \mathcal{V}\right)$ is a $(\varepsilon, d)$-regular $R$-partition.

Let $m$ be the common cluster size of $\mathcal{V}$. Let $\mathcal{H}^{\prime}=\mathcal{H} \cap K_{k}\left(G^{\prime}\right)$ and $\mathcal{U}^{\prime}=\left\{U \cap V\left(G^{\prime}\right): U \in \mathcal{U}\right\}$ the restriction of $\mathcal{U}$ to $V\left(G^{\prime}\right)$. Note that (S1) and $\varepsilon, d \ll \mu$ together imply that $\mathcal{U}^{\prime}$-partite $(\mu / 2)$-approximations of $G^{\prime}$ will be $\mathcal{U}$-partite $\mu$-approximations of $G$. Since $(G, \mathcal{H})$ is an 
$\mu$-robust $\mathcal{U}$-partite Hamilton framework, we deduce that $\left(G^{\prime}, \mathcal{H}^{\prime}\right)$ must be a $(\mu / 2)$-robust $\mathcal{U}^{\prime}$-partite Hamilton framework.

Step 2: Locating entry points. Let $\mathcal{J}$ be the $k$-graph induced by $\left(\mathcal{H}^{\prime}, \mathcal{V}\right)$. Note that since $\left(G^{\prime}, \mathcal{V}\right)$ is an $R$-partition and $\mathcal{H}^{\prime} \subseteq K_{k}\left(G^{\prime}\right)$, we have $\mathcal{J} \subseteq K_{k}(R)$. The following claim states that every vertex of $\mathcal{H}$ can be added to $\mathcal{V}$ in many ways.

Claim 7.12. There is a collection $\mathcal{F}$ of subgraphs of $\mathcal{J}$ such that

(i) $\mathcal{F}$ has at most $2^{t^{k}}$ elements,

(ii) each subgraph in $\mathcal{F}$ has at least $\gamma(r t)^{k}$ edges and

(iii) for every vertex $v \in V(G)$, there is an $\mathcal{F} \in \mathcal{F}$ such that $v$ has at least $4 \gamma m^{k}$ linked edges (in $\mathcal{H}^{\prime}$ ) in the clusters of $\mathcal{V}$ corresponding to every $K \in E(\mathcal{F})$.

Proof of the claim. Fix a vertex $v \in V(G)$. Since $(G, \mathcal{H})$ is a $\mu$-robust $\mathcal{U}$-partite $k$-uniform Hamilton framework, $v$ has at least $\mu n^{k}$ linked edges in $\mathcal{H}$. Note that removing all edges from $\mathcal{H}$ which contain a given vertex (resp. a given pair of vertices) eliminates at most $n^{k-1}$ (resp. $n^{k-2}$ ) linked edges of $v$. Since $\mathcal{H}^{\prime}=\mathcal{H} \cap K_{k}\left(G^{\prime}\right), d, \varepsilon \ll \mu$ and (S1) imply that at most $\varepsilon n^{k}+(d+\varepsilon) n^{k} \leqslant \mu n^{k} / 2$ linked edges of $v$ are destroyed in passing from $\mathcal{H}$ to $\mathcal{H}^{\prime}$. We deduce that at least $(\mu / 2) n^{k}$ of these linked edges must be contained in $\mathcal{H}^{\prime}$. We let $\mathcal{F}_{v} \subseteq E(\mathcal{J})$ be the set of edges $K$ such that $v$ has at least $4 \gamma m^{k}$ linked edges in the clusters corresponding to $K$. A standard averaging argument together with $1 / t \ll \gamma \ll \mu$ then shows that $\left|\mathcal{F}_{v}\right| \geqslant \gamma(r t)^{k}$.

Let $\mathcal{F}=\left\{\mathcal{F}_{v}: v \in V(G)\right\}$. Since each $\mathcal{F} \in \mathcal{F}$ is a subset of edges of $\mathcal{J}$, and $\mathcal{J}$ has at most $t^{k}$ edges, we have $|\mathcal{F}| \leqslant 2^{t^{k}}$, as required.

Step 3: Refining the partition. Consider a collection $\mathcal{F}$ as in Claim 7.12. Note that the only difference to property (R4) in the lemma statement is the size of $\mathcal{F}$. We will adjust this by finding a suitable refinement of $\mathcal{V}$ and then considering the corresponding blow-ups of $R, \mathcal{J}$ and $\mathcal{F}$.

Let $s$ be the least integer such that $s \geqslant t^{k-1} / \eta$ and $s \equiv 1 \bmod k$. Set $t^{*}=s t$ and note that $t^{*} \leqslant t_{1}^{*}$. Moreover, $t^{k} \leqslant \eta t^{*}$ and $t^{*} \equiv t \equiv q \bmod k$. Now consider a random refinement $\mathcal{V}^{*}$ of $\mathcal{V}$ obtained as follows. For every $1 \leqslant i \leqslant r t$, we partition $V_{i}$ into $\left\{V_{i, j}\right\}_{j=1}^{s}$ by placing each vertex of $V_{i}$ uniformly at random in one of the parts $V_{i, 1}, \ldots, V_{i, s}$. Let $m^{*}$ be the minimum cluster size of $\mathcal{V}^{*}$. Let $R^{*}$ be the graph on $r t^{*}$ vertices induced by $\left(R, \mathcal{V}^{*}\right)$. By Proposition 6.3, with probability at least $2 / 3$, we have that

$\left(\mathrm{R} 3^{\prime}\right)\left(G^{\prime}, \mathcal{V}^{*}\right)$ is a $\left(1+\varepsilon^{\prime}\right)$-balanced $\left(\varepsilon^{\prime}, d\right)$-regular $R^{*}$-partition.

Let $\mathcal{J}^{*}$ be the $s$-blow-up of $\mathcal{J}$. Let $\mathcal{F}^{*}=\left\{\mathcal{F}^{*}: \mathcal{F} \in \mathcal{F}\right\}$ where $\mathcal{F}^{*} \subseteq \mathcal{J}^{*}$ is the subgraph spanned by the edge set

$$
E\left(F^{*}\right)=\left\{\left\{\left(i_{1}, j_{1}\right), \ldots,\left(i_{k}, j_{k}\right)\right\}:\left\{i_{1}, \ldots, i_{k}\right\} \in E(\mathcal{F}) \text { and } 1 \leqslant j_{1}, \ldots, j_{k} \leqslant s\right\} .
$$

Claim 7.13. With probability at least $2 / 3$, we have:

(i) $\mathcal{F}^{*}$ has at most $2^{\eta t^{*}}$ elements,

(ii) each subgraph $\mathcal{F}^{*}$ in $\mathcal{F}^{*}$ has at least $\gamma\left(r t^{*}\right)^{k}$ edges and

(iii) for every vertex $v \in V(G)$, there is an $\mathcal{F}^{*} \in \mathcal{F}^{*}$ such that $v$ has at least $2 \gamma\left(m^{*}\right)^{k}$ linked edges (in $\left.\mathcal{H}^{\prime}\right)$ in the clusters of $\mathcal{V}^{*}$ corresponding to every $K \in E\left(\mathcal{F}^{*}\right)$.

Proof of the claim. We have $\left|\mathcal{F}^{\prime}\right|=|\mathcal{F}| \leqslant 2^{t^{k}} \leqslant 2^{\eta t^{*}}$ deterministically, by Claim 7.12 and the choice of $s$ and $t^{*}$. Analogously, for any $\mathcal{F}^{*} \in \mathcal{F}^{*}$, we have $\left|\mathcal{F}^{*}\right|=|\mathcal{F}| s^{k} \geqslant \gamma\left(r t^{*}\right)^{k}$, as required.

Now consider a vertex $v \in V$. By Claim 7.12, there is an $\mathcal{F} \in \mathcal{F}$ such that $v$ has at least $4 \gamma m^{k}$ linked edges in $\mathcal{H}^{\prime}$ in the clusters of $\mathcal{V}$ corresponding to every $K \in E(\mathcal{F})$. Given such $K$, let us write $K=\left\{i_{1}, \ldots, i_{k}\right\}$ and fix $1 \leqslant j_{1}, \ldots, j_{k} \leqslant s$. By McDiarmid's inequality [59] with probability at least $1-\exp (-\Omega(n))$ the vertex $v$ has at least $2 \gamma\left(m^{*}\right)^{k}$ linked edges in the clusters of $\mathcal{V}^{*}$ corresponding to $\left\{\left(i_{1}, j_{1}\right), \ldots,\left(i_{k}, j_{k}\right)\right\}$. Since $1 / n \ll 1 / t \ll 1 / k$, taking 
an union bound over all vertices $v \in V$, subgraphs $\mathcal{F} \in \mathcal{F}$, edges $K \in E(\mathcal{F})$ and choices of $1 \leqslant i_{1}, \ldots, i_{k} \leqslant s$, yields the desired result.

From now on, fix an outcome of $\mathcal{V}^{*}$ (and thus $m^{*}, R^{*}, \mathcal{J}^{*}$ and $\mathcal{F}^{*}$ ) satisfying item $\left(\mathrm{R} 3^{\prime}\right)$ and the properties of Claim 7.13.

Step 4: Balancing the partition. Let $\mathcal{W}$ be the $\left(t^{*}, r\right)$-sized partition of $R^{*}$ induced by $\left(\mathcal{V}^{*}, \mathcal{U}\right)$. Since $\left(G^{\prime}, \mathcal{V}^{*}\right)$ is an $R^{*}$-partition, and $\left(G^{\prime}, \mathcal{H}^{\prime}\right)$ is a $(\mu / 2)$-robust $\mathcal{U}^{\prime}$-partite Hamilton framework, it follows that $\left(R^{*}, \mathcal{J}^{*}\right)$ is a $(\mu / 4)$-robust $\mathcal{W}$-partite Hamilton framework by Proposition 7.8. Moreover, $\left(G^{\prime}, \mathcal{H}^{\prime}\right)$ is also $(\mu / 2,2 / n, \mathcal{W})$-cluster-matchable (and therefore $(\mu / 4,2 / n, \mathcal{W})$-cluster-matchable) by Proposition 7.10 .

Finally, since $\mathcal{V}^{*}$ is $\left(1+\varepsilon^{\prime}\right)$-balanced, we can delete up to $\varepsilon^{\prime} m^{*}$ vertices of each part of $\mathcal{V}^{*}$ in order to ensure that all clusters have common size $m^{*}$. We also delete these vertices from $G^{\prime}$ and $\mathcal{H}^{\prime}$. Note that the total number of vertices removed this way is bounded by $\varepsilon^{\prime} m^{*} r t^{*} \leqslant 2 \varepsilon^{\prime} n$. Since $\varepsilon \ll \varepsilon^{\prime} \ll d, G^{\prime}$ is still a U-partite $\left(2 \varepsilon^{\prime}, 2 d\right)$-approximation of $G$. As $\varepsilon^{\prime} \ll \gamma$, the last property of Claim 7.13 still holds with $\gamma\left(n /\left(r t^{*}\right)\right)^{k}$ in place of $2 \gamma\left(m^{*}\right)^{k}$. Moreover, $\left(G^{\prime}, \mathcal{V}^{*}\right)$ is still a $\left(100 \sqrt{\varepsilon^{\prime}}, d / 2\right)$-regular $R^{*}$-partition by Proposition 6.1 (Robust regular and super-regular pairs). Finally, consider $v \in V$. Note that the reallocation of vertices in the clusters corresponding to $\left\{\left(i_{1}, j_{1}\right), \ldots,\left(i_{k}, j_{k}\right)\right\}$ of $\mathcal{V}^{*}$, could have destroyed, at most, $k \varepsilon^{\prime} m^{* k} \leqslant \gamma m^{* k}$ linked edges of $v$ living in those clusters. We thus deduce that for every vertex $v \in V$, there is an $\mathcal{F} \in \mathcal{F}^{\prime}$ such that $v$ has at least $\gamma m^{* k}$ linked edges in the clusters corresponding to every edge $K \in E(\mathcal{F})$. Hence, we may finish with the parameters $100 \sqrt{\varepsilon^{\prime}}, t^{*}, t_{1}^{*}, \mathcal{V}^{*}, R^{*}, \mathcal{F}^{*}, d / 2$ playing the roles of $\varepsilon, t, t_{1} \mathcal{V}, R, \mathcal{F}, d$, respectively. This finishes Step 4 , and the proof, in the case where $(G, \mathcal{H})$ is not necessarily aperiodic or zero-free.

If $(G, \mathcal{H})$ is $\mu$-robust aperiodic or zero-free, we follow the same proof. It is easy to see in Step 1 that the property of being aperiodic or zero-free is inherited by $\left(G^{\prime}, \mathcal{H}^{\prime}\right)$. In Step 4 the property is again inherited by $\left(R^{*}, \mathcal{J}^{*}\right)$, this time by Proposition 7.8. This finishes the proof in all cases.

\section{EMBedding POWERS OF HAMilton CYClES}

In this section, we prove Theorem 3.4 (Frameworks - cycles). In fact, we show a slightly stronger result in which we do not only obtain the power of a Hamilton cycle, but we are also guaranteed that the cycle visits a predefined set of cliques. This strengthening will be used in the proof of Theorem 3.5 (Frameworks - bandwidth) later.

Theorem 8.1 (Distributed Hamiltonicity). Let $1 / n \ll \eta \ll \pi \ll \mu, 1 / k$, and let $r \in\{1, k\}$. Let $\mathcal{U}$ be an $(n, r)$-sized partition, and let $(G, \mathcal{H})$ be a $\mu$-robust $\mathcal{U}$-partite Hamilton framework. If $r=1$, suppose in addition that $(G, \mathcal{H})$ is $\mu$-robust aperiodic. Let $\mathcal{F}$ be a collection of at most $2^{\eta n}$ subgraphs of $\mathcal{H}$, which contain each at least $\mu n^{k}$ edges. Then $G$ has as a subgraph the $(k-1)$ th power of a Hamilton cycle $C$ such that $K_{k}(C)$ contains at least $\pi n$ edges of each element of $\mathcal{F}$.

The proof of Theorem 8.1 rests on the following two lemmas, whose proofs are given in Sections 8.1 and 8.2 , respectively.

Lemma 8.2 (Lemma for $G$ - cycles). Let $1 / n \ll 1 / t_{1} \ll 1 / t_{0} \ll \varepsilon \ll d \ll \mu, 1 / k$, and let $r \in\{1, k\}$. Let $\mathcal{U}$ be an $(n, r)$-sized partition, and let $(G, \mathcal{H})$ be a $\mu$-robust $\mathcal{U}$-partite Hamilton framework. Then there are $t_{0} \leqslant t \leqslant t_{1}$, a spanning subgraph $G^{\prime} \subseteq G$, a U-refining $(1+\varepsilon)$-balanced partition $\mathcal{V}$ of $G^{\prime}$ with $t$ clusters inside each cluster of $\mathcal{U}$, and a graph $R$ on vertex set $\mathcal{V}$ such that the following holds. Let $\mathcal{H}^{\prime}=\mathcal{H} \cap K_{k}\left(G^{\prime}\right)$, and let $\mathcal{J}$ be the $k$-graph induced by $\left(\mathcal{H}^{\prime}, \mathcal{V}\right)$. There also exists a spanning subgraph $\mathcal{J}^{\prime} \subseteq \mathcal{J}$ with $\Delta\left(\mathcal{J}^{\prime}\right) \leqslant k^{2}+1$, so that, for $R^{\prime}=\partial_{2} \mathcal{J}^{\prime}$, we have

(G1) $e\left(G^{\prime}\right) \geqslant(1-\mu / 2) e(G)$,

(G2) $\left(G^{\prime}, \mathcal{V}\right)$ is an $(\varepsilon, d)$-regular $R$-partition, which is $(\varepsilon, d)$-super-regular on $R^{\prime}$ and

(G3) $(R, \mathcal{J})$ is a $(\mu / 4)$-robust $\mathcal{W}$-partite Hamilton framework and $(\mu / 4,2 / n, \mathcal{W})$-clustermatchable where $\mathcal{W}$ is the $(t, r)$-sized partition induced by $(\mathcal{V}, \mathcal{U})$. 
Moreover, if $(G, \mathcal{H})$ is $\mu$-robust aperiodic, then $(R, \mathcal{J})$ is $(\mu / 4)$-robust aperiodic as well.

Lemma 8.3 (Lemma for $C$ ). Let $1 / n \ll \pi \ll 1 / t \ll \varepsilon \ll \alpha \ll \mu, 1 / k$, and let $r \in\{1, k\}$. Let $\mathcal{U}$ be an $(n, r)$-sized partition and let $G$ be a $\mathcal{U}$-partite graph. Let $\mathcal{V}$ be a U-refining $(1+\varepsilon)$ balanced partition with $t$ clusters inside each cluster of $\mathcal{U}$, and let $R$ be a graph on $\mathcal{V}$ such that $(G, \mathcal{V})$ is an $R$-partition. Let $\mathcal{W}$ be the $(t, r)$-sized partition of $R$ induced by $(\mathcal{V}, \mathcal{U})$. Suppose that $(R, \mathcal{J})$ is a $\mu$-robust $\mathcal{W}$-partite Hamilton framework and $(\mu, 2 / n, \mathcal{W})$-cluster-matchable. If $r=1$, suppose in addition that $(R, \mathcal{J})$ is aperiodic. Let $\mathcal{J}^{\prime} \subseteq \mathcal{J}$ be a spanning subgraph with $\Delta\left(\mathcal{J}^{\prime}\right) \leqslant k^{2}+1$.

Then there is a size-compatible (with $\mathcal{V}$ ) vertex partition $\mathcal{X}$ of the vertex set of the $(k-1)$ th power of a cycle $C$, a family of subsets $\tilde{X}=\left\{\tilde{X}_{i}\right\}_{i=1}^{t}$ of $V(C)$, and families of $(k-1)$ th powers of paths $\left\{P_{K}\right\}_{K \in E(\mathcal{J})}$ which are subgraphs of $C$ such that

$(\mathrm{C} 1)(C, X)$ is an $R$-partition,

(C2) $\tilde{X}$ is an $\left(\alpha, R^{\prime}\right)$-buffer for $(C, X)$ where $R^{\prime}=\partial_{2} \mathcal{J}^{\prime}$,

(C3) for each $K \in E(\mathcal{J}), P_{K}$ has length $\pi n$ and $P_{K} \subseteq C\left[\bigcup_{i \in K} X_{i}\right]$, and

(C4) the paths $\left\{P_{K}\right\}_{K \in E(\mathcal{J})}$ are pairwise vertex-disjoint.

Assuming the validity of these two lemmas, we now prove the main result of this section.

Proof of Theorem 8.1. We introduce $\eta, \pi, \varepsilon, \varepsilon^{\prime}, d, \alpha>0$ and $t_{1}, t_{0} \in \mathbb{N}$ to establish the following constant hierarchy

$$
1 / n \ll \eta \ll \pi \ll 1 / t_{1} \ll 1 / t_{0} \ll \varepsilon \ll \varepsilon^{\prime} \ll d, \alpha \ll \mu \ll 1 / k .
$$

More precisely, given $k \geqslant 2$, we can assume that $\mu$ small enough for the upcoming inequalities. Next, choose $d>0$ to satisfy Lemma 8.2. For $k$ and $\mu / 4$, choose $\alpha>0$ to satisfy Lemma 8.3. We select $\varepsilon^{\prime} \leqslant \min \left\{\mu / 2, d\left(1-(3 / 4)^{1 /(k-1)}\right)\right\}$ which is also sufficiently small so that Proposition 6.4 is valid when the input is $k$ and $d$. Given $k, d, \varepsilon^{\prime}$, Proposition 6.4 also outputs a suitable $\varepsilon_{0}$ (in place of $\varepsilon$ ). Let $\zeta=1 / 2$. Then select $\varepsilon_{1}, \varrho>0$, so that Lemma 6.7 (Blow-Up Lemma) can be applied with $\Delta=\Delta_{J}=2(k-1), \Delta_{R^{\prime}}=k^{3}, \kappa=2$, $\alpha, \zeta$ and $d$. Select also $\varepsilon_{2}$ small enough to serve as input for Lemma 8.2 (given $\mu, k, d$ ) and $\varepsilon_{3}$ to serve as an input for Lemma 8.3 (given $\mu / 4, k, 2 \alpha$ in place of $\mu, k, \alpha$ ), and let $\varepsilon=\min \left\{\varepsilon_{0}, \varepsilon_{1}, \varepsilon_{2}, \varepsilon_{3}, \mu / 4,1 / 2\right\}$.

Choose $t_{0}$ that satisfies Lemmas 8.2 and 8.3 with the above constants. Given this $t_{0}$, Lemma 8.2 yields a $t_{1}$. Handing $t_{1}$ to Lemma 8.3 (in place of $t$ ) outputs an appropriate $\pi_{1}$ (in place of $\pi$ ). We give $k$ and $\mu /\left(4 t_{1}^{k}\right)$ as an input (in place of $k$ and $\gamma$ ) to Proposition 7.1 (Distributed matching) and obtain $\pi_{2}$ as an output (in place of $\pi$ ). We let $\pi=\min \left\{\pi_{1}^{1 / 2} /(3 k), \pi_{2},(\varrho /(2 k t))^{2}\right\}$. With this choice of $\pi$, Proposition 7.1 yields $\eta$. Finally, we choose $n$ large enough for Lemmas 6.7, 8.2 and 8.3 and Proposition 7.1 are satisfied (together with the above choices).

Now suppose that $G, \mathcal{U}$ and $\mathcal{F}$ meet the conditions of Theorem 8.1. Namely, $(G, \mathcal{H})$ is a $\mu$ robust $\mathcal{U}$-partite Hamilton framework where $\mathcal{U}$ is an $(n, r)$-sized partition of $G$. In addition, if $r=1$ then $(G, \mathcal{H})$ is $\mu$-robust aperiodic. Also $\mathcal{F}$ is a collection of at most $2^{\eta n}$ subgraphs of $\mathcal{H}$, which contain each at least $\mu n^{k}$ edges. We have to show that $G$ has a tight Hamilton cycle $C$, which contains at least $\pi n$ edges of each element of $\mathcal{F}$.

Step 1: Applying the Lemma for $G$ - cycles. By Lemma 8.2 there are $t_{0} \leqslant t \leqslant t_{1}$, a spanning subgraph $G^{\prime} \subseteq G$, a $\mathcal{U}$-refining $(1+\varepsilon)$-balanced partition $\mathcal{V}=\left\{V_{i}\right\}_{i=1}^{r t}$ of $G^{\prime}$ with $t$ clusters in each part of $\mathcal{U}$, a graph $R$ on $\mathcal{V}$ such that the following holds. Let $\mathcal{H}^{\prime}=\mathcal{H} \cap K_{k}\left(G^{\prime}\right)$, and let $\mathcal{J}$ be the $k$-graph induced by $\left(\mathcal{H}^{\prime}, \mathcal{V}\right)$. There also exists a spanning subgraph $\mathcal{J}^{\prime} \subseteq \mathcal{J}$ with $\Delta\left(\mathcal{J}^{\prime}\right) \leqslant k^{2}+1$, so that, for $R^{\prime}=\partial_{2} \mathcal{J}^{\prime}$, we have

(G1) $e\left(G^{\prime}\right) \geqslant(1-\mu / 2) e(G)$,

(G2) $\left(G^{\prime}, \mathcal{V}\right)$ is an $(\varepsilon, d)$-regular $R$-partition, which is $(\varepsilon, d)$-super-regular on $R^{\prime}$, and

(G3) $(R, \mathcal{J})$ is a $(\mu / 4)$-robust $\mathcal{W}$-partite Hamilton framework and $(\mu / 4,2 / n, \mathcal{W})$-clustermatchable where $\mathcal{W}$ is the $(t, r)$-sized partition induced by $(\mathcal{V}, \mathcal{U})$. 
Moreover, if $(G, \mathcal{H})$ is $\mu$-robust aperiodic, then $(R, \mathcal{J})$ is $(\mu / 4)$-robust aperiodic as well. Note that $\Delta\left(R^{\prime}\right) \leqslant(k-1)\left(k^{2}+1\right) \leqslant k^{3}$.

Step 2: Applying the Lemma for C. Next, we apply Lemma 8.3 (with $\pi_{1}$ and $2 \alpha$ in place of $\pi$ and $\alpha$ ) to obtain a size-compatible (with $\mathcal{V}$ ) vertex partition $\mathcal{X}=\left\{X_{i}\right\}_{i=1}^{r t}$ of the vertex set of the $(k-1)$ th power of a cycle $C$, a family $\tilde{X}=\left\{\tilde{X}_{i}\right\}_{i=1}^{r t}$, and a family of $(k-1)$ th powers of paths $\left\{P_{K}\right\}_{K \in E(\mathcal{J})}$ which are subgraphs of $C$ such that

(C1) $(C, X)$ is an $R$-partition,

(C2) $\tilde{X}$ is an $\left(2 \alpha, R^{\prime}\right)$-buffer for $(C, X)$,

(C3) for each $K \in E(\mathcal{J}), P_{K}$ has length $\pi_{1} n$ and $P_{K} \subseteq C\left[\bigcup_{i \in K} X_{i}\right]$, and

(C4) the paths $\left\{P_{K}\right\}_{K \in E(\mathcal{J})}$ are pairwise vertex-disjoint.

Step 3: Fixing representatives of $\mathcal{F}$. Next, we find a matching $M \subseteq \mathcal{H}^{\prime}$ of bounded size, which contains many edges of every subgraph of $\mathcal{F}$, as follows. Fix a subgraph $\mathcal{F} \in \mathcal{F}$, and let $\mathcal{F}_{1}=\mathcal{F} \cap \mathcal{H}^{\prime}$. Since $\mathcal{F}$ has at least $\mu n^{k}$ edges and $e\left(G^{\prime}\right) \geqslant(1-\mu / 2) e(G)$, it follows that $\mathcal{F}_{1}$ retains at least $(\mu / 2) n^{k}$ edges. (Here we used that each edge of $G$ is in at most $n^{k-2}$ edges of $\mathcal{H}$.) Moreover, by the pigeonhole principle and since $\mathcal{J}$ has at most $t^{k}$ edges, there is an edge $K_{\mathcal{F}}$ in $\mathcal{J}$ such that there are at least $(\mu / 2)(n / t)^{k}$ edges $\mathcal{F}_{2} \subseteq \mathcal{F}_{1}$ which are partite to the clusters of $K_{\mathcal{F}}$.

Let us call an edge $e$ of $\mathcal{F}_{2}$ typical, if every $k-1$ vertices of $e$ share at least $(d-\varepsilon)^{k-1} n / t$ common neighbours in $G^{\prime}$ in the cluster of $K_{\mathcal{F}}$ that they miss. By Proposition 6.4 (Joint degrees in regular pairs) all but at most $\varepsilon^{\prime}(n / t)^{k}$ edges of $\mathcal{F}_{2}$ are typical. We write $\mathcal{F}_{3} \subseteq \mathcal{F}_{2}$ for the subgraph of typical edges of $\mathcal{F}_{2}$. From the choice of $\varepsilon^{\prime}$ and $t \leqslant t_{1}$ we note that $\left|\mathcal{F}_{3}\right| \geqslant\left|\mathcal{F}_{2}\right|-\varepsilon^{\prime}(n / t)^{k} \geqslant(\mu / 4)(n / t)^{k} \geqslant(\mu / 4)\left(n / t_{1}\right)^{k}$. Let $\mathcal{F}^{\prime}=\left\{\mathcal{F}_{3}: \mathcal{F} \in \mathcal{F}\right\}$. It follows by Proposition 7.1 (Distributed matching) applied with $\eta, \pi$ and $\mu /\left(4 t_{1}^{k}\right)$ playing the role of $\gamma$ that there is a matching $M \subseteq \mathcal{H}^{\prime}$ of size at most $\sqrt{\pi} n$ such that $M$ has at least $\pi n$ edges of every subgraph $\mathcal{F}_{3} \in \mathcal{F}^{\prime}$.

For each $e \in M$, let $K$ be the edge of $R$ whose clusters of $\mathcal{V}$ contain $e$, and select a $(k-1)$ th power of path $P_{e} \subseteq P_{K} \subseteq C$ of length $3 k$. This can be done in such a way that the family $\left\{P_{e}\right\}_{e \in M}$ consists of pairwise vertex-disjoint powers of paths. Indeed, by the choice of $\pi$, we have $\pi_{1} n \geqslant 3 k \pi^{1 / 2} n$; thus (C3) implies that for each $K \in E(\mathcal{J})$, the associated power of path $P_{K}$ has length at least $3 k \pi^{1 / 2} n \geqslant 3 k e(M)$, where the latter inequality follows from $e(M) \leqslant \sqrt{\pi} n$.

Step 4: Applying the Blow-Up Lemma. We finish the proof with an application of the BlowUp Lemma. Recall that $\left\{\tilde{X}_{i}\right\}_{i=1}^{r t}$ is a $\left(2 \alpha, R^{\prime}\right)$-buffer for $C$. To finish, we define a subgraph $C^{\prime} \subseteq C$, a family of image restrictions $\mathcal{J}=\left\{I_{x}\right\}_{x \in V(C)}$ and a family of restricting vertices $\mathcal{J}=\left\{J_{x}\right\}_{x \in V(C)}$. The idea is to 'crop out' the middle part of each power of a path $P_{e}$, and ensure that the gap will be mapped on the edge $e \in E(M)$ via an image restriction.

More precisely, for every edge $e=\left\{v_{1}, \ldots, v_{k}\right\}$ in $M$ whose corresponding power of a path $P_{e} \subseteq C$ is given by $P_{e}=\left(x_{1}, \ldots, x_{k}, y_{1}, \ldots, y_{k}, x_{k+1}, \ldots, x_{2 k}\right)$, let $Y_{e}=\left\{y_{1}, \ldots, y_{k}\right\} \subseteq V\left(P_{e}\right)$. We define $C^{\prime} \subseteq C$ by $C^{\prime}=C-\bigcup_{e \in E(M)} Y_{e}$. Next, we define our restriction pair. For each $e \in E(M)$ and $x_{j} \in V\left(P_{e}\right)$, set $J_{x_{j}}=e \backslash\left\{v_{j}\right\}$ and $I_{x_{j}}=\bigcap_{v \in e} N_{G^{\prime}}(v) \cap V_{i}$ for each $1 \leqslant j \leqslant 2 k$ and $x_{j} \in X_{i}$. Note that since $e$ is typical, we have $\left|I_{x_{j}}\right| \geqslant\left(d-\varepsilon^{\prime}\right)^{k-1} n / t \geqslant \zeta d^{k-1}\left|V_{i}\right|$, where in the inequality we used that $\zeta=1 / 2$, that $\mathcal{V}$ is $(1+\varepsilon)$-balanced, $\varepsilon \leqslant 1 / 2$ and the choice of $\varepsilon^{\prime}$. For all other $x \in V(C) \backslash V\left(C^{\prime}\right)$, we simply set $J_{x}=\emptyset$ and $I_{x}=V_{i^{\prime}}$ where $x \in X_{i^{\prime}}$. The total number of image restricted vertices overall is $2|v(M)| \leqslant 2 k \pi^{1 / 2} n$, thus certainly there are at most $2 k \sqrt{\pi} n \leqslant \varrho n / t$ image restricted vertices $X_{i}^{*}$ in each $X_{i}$ (the inequality follows from the choice of $\pi)$. It follows that $\mathcal{J}$ and $\mathcal{J}$ form a $\left(\varrho, \zeta, \Delta, \Delta_{J}\right)$-restriction pair.

Finally, we apply the Blow-Up Lemma (Lemma 6.7) with $G=G^{\prime}-V(M)$ and $\mathcal{H}=C^{\prime}$ together with the above chosen parameters (note that $\kappa \geqslant 1+\varepsilon$ ) to obtain an embedding $\psi: V\left(C^{\prime}\right) \rightarrow V(G)$ such that $\psi(x) \in I_{x}$ for each $x \in V(\mathcal{H})$. It follows that $G$ contains the $(k-1)$ th power of a Hamilton cycle $C^{\prime \prime}$, where $K_{k}\left(C^{\prime \prime}\right)$ contains all edges of $M$. By the 
construction of $M$, we deduce $K_{k}\left(C^{\prime \prime}\right)$ contains at least $\pi n$ edges of each element of $\mathcal{F}$, as required.

8.1. Proof of the Lemma for $G$ - cycles. In the following, we show Lemma 8.2.

Proof of Lemma 8.2. We establish the following constant hierarchy for the remainder of the proof.

$1 / n \ll 1 / t_{1} \ll 1 / t_{0} \ll \varepsilon \ll \varepsilon^{\prime} \ll \varepsilon^{\prime \prime} \ll d^{\prime \prime} \ll d^{\prime} \ll d \ll \eta \ll \pi \ll \gamma \ll \mu, 1 / k . \quad\left(\ll_{G}\right)$

In particular, we choose $\gamma, \pi, \eta, d, \varepsilon, t_{0}, t_{1}, n$ so that we can apply Proposition 7.1 (with $r t$ and $\gamma$ playing the role of $n$ and $\gamma$ ), Proposition 6.2 (with $t_{0} \leqslant t \leqslant t_{1}$ and $\Delta_{R^{\prime}}=k^{3}$ ) and Lemma 7.11. We also choose these constants such that the various inequalities along the proof are satisfied. Now let $\mathcal{U}$ be an $(n, r)$-sized partition, and let $(G, \mathcal{H})$ be a $\mu$-robust $\mathcal{U}$-partite $k$-uniform Hamilton framework.

Step 1: Establishing a regular partition. We begin by applying Lemma 7.11 (Regular partition with entry points) to $(G, \mathcal{H})$ and $\mathcal{U}$. Hence, there are $t_{0} \leqslant t \leqslant t_{1}$, a subgraph $G^{\prime} \subseteq G$ with a balanced $\mathcal{U}$-refining partition $\mathcal{V}=\left\{V_{i}\right\}_{i=1}^{r t}$ with $t$ clusters in each part of $\mathcal{U}$, a graph $R$ on $\mathcal{V}$, such that

(R1) $G^{\prime}$ is an $(\varepsilon, 4 d)$-approximation of $G$ and

(R2) $\left(G^{\prime}, \mathcal{V}\right)$ is a $(\varepsilon, d)$-regular $R$-partition with common cluster size $m$.

Moreover, if we let $\mathcal{H}^{\prime}=\mathcal{H} \cap K_{k}\left(G^{\prime}\right)$, we let $\mathcal{J}$ be the $k$-graph induced by $\left(\mathcal{H}^{\prime}, \mathcal{V}\right)$, and we let $\mathcal{W}$ be the $(t, r)$-sized partition induced by $(\mathcal{V}, \mathcal{U})$, then we also have that

(R3) $(R, \mathcal{J})$ is a $(\mu / 4)$-robust $\mathcal{W}$-partite Hamilton framework,

(R4) $(R, \mathcal{J})$ is $(\mu / 4,2 / n, \mathcal{W})$-cluster-matchable, and

(R5) if $r=1,(R, \mathcal{J})$ is a $(\mu / 4)$-robust aperiodic Hamilton framework.

Moreover, there is a collection $\mathcal{F}$ of subgraphs of $\mathcal{J}$ such that

(R6) (i) $\mathcal{F}$ has at most $2^{\eta t}$ elements,

(ii) each subgraph in $\mathcal{F}$ has at least $\gamma(r t)^{k}$ edges,

(iii) for every vertex $v \in V(G)$, there is a $\mathcal{F} \in \mathcal{F}$ such that $v$ has at least $\gamma(n /(r t))^{k}$ linked edges in $\mathcal{H}^{\prime}$ in the clusters of $\mathcal{V}$ corresponding to every $K \in E(\mathcal{F})$.

We then apply Proposition 7.1 (Distributed matching) to $\mathcal{J}$ and $\mathcal{F}$. By the constant hierarchy $\left(\ll_{G}\right)$, we obtain

$\left(\mathrm{R} 4^{\prime}\right)$ a matching $\mathcal{M} \subseteq \mathcal{J}$ of size at most $\sqrt{\pi} r t \leqslant(\mu /(8 k)) t$ such that $\mathcal{M}$ has at least $\pi r t$ edges of every subgraph $\mathcal{F} \in \mathcal{F}$.

Step 2: Finding $R^{\prime}$. We start by selecting a subhypergraph $\mathcal{Q} \subseteq \mathcal{J}-V(\mathcal{M})$ of bounded maximum degree. Recall that $(R, \mathcal{J})$ is a $(\mu / 4)$-robust $\mathcal{W}$-partite Hamilton framework by item (R3). Since $\mathcal{M}$ has size at most $\mu t /(8 k)$, we deduce $|V(\mathcal{M})| \leqslant \mu t / 8$. Therefore, we get that $(R-V(\mathcal{M}), \mathcal{J}-V(\mathcal{M}))$ is a $(\mu / 8)$-robust $\mathcal{W}^{\prime}$-partite Hamilton framework, where $\mathcal{W}^{\prime}$ is obtained by deleting $V(\mathcal{M})$ from the parts of $\mathcal{W}$. In particular, the $k$-graph $\mathcal{J}-V(\mathcal{M})$ must have a perfect fractional matching. By Lemma 7.7 (Bounded degree cover), $\mathcal{J}-V(\mathcal{M})$ contains a spanning subhypergraph $\mathcal{Q} \subseteq \mathcal{J}-V(\mathcal{M})$ such that each vertex of $\mathcal{Q}$ is in at most $k^{2}+1$ edges, as desired.

Let $\mathcal{J}^{\prime}=\mathcal{M} \cup \mathcal{Q}$ and $R^{\prime}=\partial_{2} \mathcal{J}^{\prime}$. Note that $\Delta\left(R^{\prime}\right) \leqslant\left(k^{2}+1\right)(k-1) \leqslant k^{3}$.

Step 3: Super-regularising $R^{\prime}$. Next, we find a family of subsets in the partition $\mathcal{V}$, which has suitable super-regular properties. We apply Proposition 6.2 (Super-regularising $R^{\prime}$ ) with $\varepsilon$, $d$ and $R^{\prime}, G^{\prime}, k^{3}$ playing the role of $R^{\prime}, G, \Delta_{R^{\prime}}$ respectively. This gives a balanced family $\mathcal{V}^{\prime}=\left\{V_{i}^{\prime}\right\}_{i=1}^{t}$ of subsets $V_{i}^{\prime} \subseteq V_{i}$ of size $m^{\prime}=\lfloor(1-\sqrt{\varepsilon}) m\rfloor$ such that $\left(G^{\prime}, \mathcal{V}^{\prime}\right)$ is an $(2 \varepsilon, d)$ regular $R$-partition, which is $(2 \varepsilon, d)$-super-regular on $R^{\prime}$. Let $V_{0}=V(G) \backslash \bigcup_{i=1}^{r t} V_{i}^{\prime}$ denote the set of 'exceptional vertices'. Note that $\left|V_{0}\right| \leqslant \varepsilon r n+r t\lceil\sqrt{\varepsilon} m\rceil \leqslant 3 \sqrt{\varepsilon} r n$, where the first inequality follows from property (R1) and the choice of $\mathcal{V}^{\prime}$.

In passing from $\mathcal{V}$ to $\mathcal{V}^{\prime}$, no more than $\lceil\sqrt{\varepsilon} m\rceil \leqslant 2 \sqrt{\varepsilon} m$ vertices were removed in each cluster, and each of these removals can affect at most $m^{k-1}$ of the linked edges of a vertex 
which are located in a edge $K \in E(\mathcal{M})$. These observations, together with properties (R6), $\left(\mathrm{R} 4^{\prime}\right)$ and $\varepsilon \ll \gamma$, imply that

$\left(\mathrm{R} 5^{\prime}\right)$ for every $v \in V(\mathcal{H})$, there are at least $\pi r t$ edges $K \in E(\mathcal{M})$, such that $v$ has at least $(\gamma / 2)\left(m^{\prime}\right)^{k}$ linked edges in $\mathcal{H}$ in the clusters of $\mathcal{V}^{\prime}$ corresponding to $K$.

Step 4: The return of the exceptional vertices. To finish, we have to add the exceptional vertices $V_{0}$ back to the partition $\mathcal{V}^{\prime}$. We will use the linked edges for this, for which we introduce some terminology. Recall that a linked edge of $v$ corresponds to an edge $e \in \mathcal{H}$ such that there exists $f \in E(\mathcal{H})$ with $v \in f$ and $|e \cap f|=k-1$. Say the unique vertex in $e \backslash f$ is the representative of $v$ in $e$. Fix $v \in V_{0}$, and some $K \in E(\mathcal{M})$ such that $v$ has at least $(\gamma / 2)\left(m^{\prime}\right)^{k}$ linked edges which are located in the $k$ clusters of $\mathcal{V}^{\prime}$ corresponding to $K$. Since $v$ has at least $(\gamma / 2)\left(m^{\prime}\right)^{k}$ linked edges in the clusters of $\mathcal{V}^{\prime}$ corresponding to $K$, by averaging there exists $i_{v} \in V(K)$ such that $v$ has at least $(\gamma / 2 k)\left(m^{\prime}\right)^{k}$ linked edges whose representative lies in the cluster $V_{i_{v}}^{\prime}$. Say such $i_{v}$ is a representative index of $v$. In particular, note that this implies $v$ has at least $\gamma m^{\prime} /(2 k)$ neighbours in each of the clusters corresponding to the vertices of $K$ which are not $V_{i_{v}}^{\prime}$.

The previous argument and property $\left(\mathrm{R} 5^{\prime}\right)$ implies that for each $v \in V_{0}$ there are at least $\pi r t$ representative indices. Hence, we can assign, for each vertex $v \in V_{0}$, a representative index $i_{v}$ in such a way that no index is assigned to more than $\left|V_{0}\right| /(\pi r t) \leqslant(3 \sqrt{\varepsilon} n) /(\pi r t) \leqslant$ $\varepsilon^{\prime \prime} n /(r t)$ vertices of $V_{0}$, where the inequality follows from the constant hierarchy $\left(\ll_{G}\right)$.

For each $1 \leqslant i \leqslant r t$, let $V_{i}^{\prime \prime \prime}$ be obtained from $V_{i}^{\prime \prime}$ by adding the vertices $v$ from $V_{0}$ such that $i=i_{v}$, and let $\mathcal{V}^{\prime \prime \prime}=\left\{V_{i}^{\prime \prime}: 1 \leqslant i \leqslant r t\right\}$. Note that $\mathcal{V}^{\prime \prime \prime}$ partitions $V(G)$. For each $v \in V_{0}$, write $K_{v}$ for the edge $K \in E(\mathcal{M})$ which contains the representative index $i_{v}$. Let $G^{\prime \prime}$ be the $k$-graph obtained from $G^{\prime}$ by deleting, for every vertex $v \in V_{0}$, all edges emanating from $v$ which are not in the clusters corresponding to $K_{v} \backslash\left\{i_{v}\right\}$. As argued before, $v$ has at least $(\gamma / 2 k) m^{\prime}$ neighbours in each of the clusters corresponding to the vertices of $K_{v} \backslash\left\{i_{v}\right\}$. It follows by the constant hierarchy $\left(\ll_{G}\right)$, the choice of $R^{\prime}$ and another application of Proposition 6.1 yields that $\left(G^{\prime \prime}, \mathcal{V}^{\prime \prime}\right)$ is a $\left(1+\varepsilon^{\prime \prime}\right)$-balanced $\left(\varepsilon^{\prime \prime}, d^{\prime \prime}\right)$-regular $R$-partition, which is $\left(\varepsilon^{\prime \prime}, d^{\prime \prime}\right)$-superregular on $R^{\prime}$. Moreover, $e\left(G^{\prime \prime}\right) \geqslant(1-\mu / 2) e(G)$ since $\left|V_{0}\right| \leqslant \varepsilon n$, property $(\mathrm{G} 1)$ and $\varepsilon, d \ll \mu$. Hence, we may finish the proof with $G^{\prime \prime}, \mathcal{V}^{\prime \prime}, \varepsilon^{\prime \prime}, d^{\prime \prime}$ playing the roles of $G^{\prime}, \mathcal{V}, \varepsilon, d$.

\subsection{Proof of the Lemma for $C$.}

Proof of Lemma 8.3. We establish the following hierarchy.

$$
1 / n \ll \varrho \ll \pi \ll 1 / t \ll \varepsilon \ll \alpha \ll \mu, 1 / k .
$$

Now, consider $\mathcal{U}, G, \mathcal{V}, R, \mathcal{J}, \mathcal{J}^{\prime}$ as in the statement. It will be convenient to formulate the proof in terms of tight cycles instead of powers of cycles. Denote by $\mathcal{J}^{*}$ the $(\mathcal{J}, \mathcal{V})$ blow-up $k$-graph, where the $i$ th vertex of $\mathcal{J}$ corresponds to a set of size $\left|V_{i}\right|$. Let $\mathcal{X}$ be the corresponding, size-compatible with $\mathcal{V}$, vertex partition of $V\left(\mathcal{J}^{*}\right)$. The statement of the lemma follows if $\mathcal{J}^{*}$ has a tight Hamilton cycle, which satisfies (the hypergraph analogues of) properties (C1) to (C3).

Step 1: Sketching a cycle. To begin, we set up a (comparatively) short tight cycle $C_{1}$ in $\mathcal{J}^{*}$, which 'visits' every edge of $\mathcal{J}$ and leaves the number of uncovered vertices in $\mathcal{J}^{*}$ divisible by $k$. The latter is trivial when $r=k$, because every tight cycle will satisfy that property; for $r=1$, we ensure this condition using the aperiodicity of $(R, \mathcal{J})$.

Claim 8.4. There is a tight cycle $C_{1} \subseteq \mathcal{J}^{*}$ which contains a tight path $P_{e}$ of length $k$ for every edge $e \in E(R)$. Moreover, $C_{1}$ has length at most $(r t)^{2 k}$ and $\left|V\left(\mathcal{J}^{*}\right) \backslash V\left(C_{1}\right)\right|$ is divisible by $k$.

Proof. Recall that $(R, \mathcal{J})$ is a Hamilton framework. In particular, $\mathcal{J}$ is tightly connected.

We first assume that $r=k$. By Proposition 7.3 (Closed tight walk visiting all edges) there exists a closed tight walk $W$ in $\mathcal{J}$ of length at most $k^{2}(r t)^{k}+k\left(\begin{array}{c}r t \\ k\end{array}\right)(r t)^{k} \leqslant(r t)^{2 k}$. Since $\mathcal{J}$ is $k$-partite, $W$ must have length divisible by $k$. Since a closed tight walk is a homomorphism 
of a tight cycle and $1 / n \ll 1 / t$, it follows immediately that we can lift the walk $W$ in $\mathcal{J}$ to a tight cycle $C_{1}$ in $\mathcal{J}^{*}$ of the same length. By construction, $C_{1}$ includes the required paths $P_{e}$.

Now, consider the case $r=1$. By assumption, we also have that $(R, \mathcal{J})$ is an aperiodic Hamilton framework, and thus we get that $\mathcal{J}$ contains a closed tight walk of length coprime to $k$. Now, Proposition 7.3 yields a closed tight walk in $\mathcal{J}$, which visits every edge of $\mathcal{J}$, has length at most $k^{2} t^{k}+k\left(\begin{array}{l}t \\ k\end{array}\right) t^{k} \leqslant t^{2 k}$, and in addition we can assume the length of the walk is congruent to $n$ modulo $k$. We then turn the walk in $\mathcal{J}$ into a tight cycle in $\mathcal{J}^{*}$ as before.

Let us fix a cycle $C_{1}$ as in the claim and define $\mathbf{w}_{1} \in \mathbb{N}^{E(\mathcal{J})}$ to be the vector that counts, for every $e \in E(\mathcal{J})$, the number of edges of $C_{1}$ that are contained in the clusters of $X$ corresponding to $e$.

Step 2: Allocations. It will be convenient to formulate the following allocations in terms of matrices. Let $A=A_{\mathcal{J}} \in \mathbb{N}^{V(\mathcal{J}) \times E(\mathcal{J})}$ be the incidence matrix of $\mathcal{J}$. Let $\mathbf{b} \in \mathbb{N}^{V(\mathcal{J})}$ count the vertices of each cluster of $\mathcal{X}$, that is $\mathbf{b}(i)=\left|X_{i}\right|$. Note that $\mathcal{V}$ is $(1+\varepsilon)$-balanced, thus the cluster sizes are between $m$ and $(1+\varepsilon) m$ for some integer $m$. Our goal is now to find $\mathbf{w} \in \mathbb{N}^{E(\mathcal{J})}$ such that

(i) $A\left(\mathbf{w}_{1}+\mathbf{w}\right)=\mathbf{b}$,

(ii) $\mathbf{w}(e) \geqslant 4 \pi n$ for every edge $e \in E(\mathcal{J})$,

(iii) $\mathbf{w}(e) \geqslant 3 \alpha m$ for every edge $e \in E\left(\mathcal{J}^{\prime}\right)$.

We will find $\mathbf{w}$ as the sum of four vectors $\mathbf{w}_{2}, \ldots, \mathbf{w}_{5}$. First, we note that $\left\|A \mathbf{w}_{1}\right\|_{\infty} \leqslant t^{2 k} \leqslant$ $\alpha m$. We begin by choosing $\mathbf{w}_{2} \in \mathbb{N}^{E(\mathcal{J})}$ and $\mathbf{w}_{3} \in \mathbb{N}^{E(\mathcal{J})}$ as follows. We let $\mathbf{w}_{2}(e)=\lceil 3 \alpha m\rceil$ for every edge $e \in E\left(\mathcal{J}^{\prime}\right)$ and 0 otherwise, and we let $\mathbf{w}_{3}(e)=\lceil 5 \pi n\rceil$ for every edge $e \in E(\mathcal{J})$. Note that $\left\|A \mathbf{w}_{2}\right\|_{\infty} \leqslant\left(k^{2}+1\right)\lceil 3 \alpha m\rceil \leqslant 4 k^{2} \alpha m$. This is because every vertex is in at most $k^{2}+1$ edges of $\mathcal{J}^{\prime}$ (by $\Delta\left(\mathcal{J}^{\prime}\right) \leqslant k^{2}+1$ ), and the last inequality follows from the hierarchy $\left(\ll_{C}\right)$. On the other hand, every vertex is in at most $t^{k-1}$ edges of $\mathcal{J}$, thus $\left\|A \mathbf{w}_{3}\right\|_{\infty} \leqslant\lceil 5 \pi n\rceil t^{k-1} \leqslant \alpha m$, where again we used the hierarchy $\left(\ll_{C}\right)$.

Next, we allocate most of the remaining vertices.

Claim 8.5. There is $\mathbf{w}_{4} \in \mathbb{N}^{E(\mathcal{J})}$ such that $0 \leqslant \mathbf{b}-A\left(\mathbf{w}_{1}+\mathbf{w}_{2}+\mathbf{w}_{3}+\mathbf{w}_{4}\right) \leqslant \varrho n$.

Proof of the claim. Let $\mathbf{w}^{\prime}=\mathbf{w}_{1}+\mathbf{w}_{2}+\mathbf{w}_{3}$ and note that $\left\|A \mathbf{w}^{\prime}\right\|_{\infty} \leqslant\left\|A \mathbf{w}_{1}\right\|_{\infty}+\left\|A \mathbf{w}_{2}\right\|_{\infty}+$ $\left\|A \mathbf{w}_{3}\right\|_{\infty} \leqslant\left(4 k^{2}+2\right) \alpha m$. Let $X^{\prime}$ be obtained from $\mathcal{X}$, by deleting, for every $K \in E(\mathcal{J})$, an amount of $\mathbf{w}^{\prime}(K)$ vertices of every cluster corresponding to $K$. Note that, for every $W \in \mathcal{W}$, the number of elements in clusters of $X^{\prime}$ with index in $W$ is the same. For $r=1$, this is trivial. For $r=k$, it follows because $\mathcal{J}$ is $\mathcal{W}$-partite and because the property was true for $\mathcal{V}$, which is size-compatible with $X$.

Since $\left(4 k^{2}+2\right) \alpha(1+\mu)+\varepsilon \leqslant \mu / 2$, we deduce $X^{\prime}$ is $(1+\mu / 2)$-balanced. Since $(R, \mathcal{J})$ is $(\mu / 2,2 / n, \mathcal{W})$-cluster-matchable, the $\left(\mathcal{J}, X^{\prime}\right)$-blow-up contains a matching $\mathcal{M}$ that covers all but at most $2+t^{k-1} \leqslant \varrho n$ vertices in each cluster of $X^{\prime}$, the inequality following from $\left(\ll_{C}\right)$. We can then define $\mathbf{w}_{4} \in \mathbb{N}^{E(\mathcal{J})}$ by counting, for each $e \in E(\mathcal{J})$, the number of $K$-edges of $\mathcal{M}$ for each $K \in E(\mathcal{J})$. (Meaning those edges $e$, which have one vertex in each of the clusters of $K$.)

Let $\mathbf{w}_{4}$ as in the claim. Finally, we complete the allocation by finding $\mathbf{w}_{5}$.

Claim 8.6. There is $\mathbf{w}_{5} \in \mathbb{Z}^{E(\mathcal{J})}$ with $\left\|\mathbf{w}_{5}\right\|_{\infty} \leqslant \pi n$ such that $A\left(\mathbf{w}_{1}+\mathbf{w}_{2}+\mathbf{w}_{3}+\mathbf{w}_{4}+\mathbf{w}_{5}\right)=\mathbf{b}$.

Proof of the claim. Set $\mathbf{c}=\mathbf{b}-A\left(\mathbf{w}_{1}+\mathbf{w}_{2}+\mathbf{w}_{3}+\mathbf{w}_{4}\right)$. By the choice of $\mathbf{w}_{4}$, we have $\|\mathbf{c}\|_{1} \leqslant t\|\mathbf{c}\|_{\infty} \leqslant t \varrho n$ and $\|\mathbf{c}\|_{1} \equiv 0 \bmod k$, where the last part follows by the choice of $\mathbf{w}_{1}$. Fix an arbitrary edge $e_{0} \in E(\mathcal{J})$ and define $\mathbf{w}_{5}^{\prime} \in \mathbb{N}^{E(\mathcal{J})}$ by setting $\mathbf{w}_{5}^{\prime}\left(e_{0}\right)=\|\mathbf{c}\|_{1} / k$ and 0 everywhere else. Then let $\mathbf{c}^{\prime}=\mathbf{c}-A \mathbf{w}_{5}^{\prime}$. Note that $\left\|\mathbf{c}^{\prime}\right\|_{\infty} \leqslant\|\mathbf{c}\|_{\infty}+\|\mathbf{c}\|_{1} \leqslant \varrho n+t \varrho n \leqslant 2 t \varrho n$. Note also that $\sum_{i \in W} \mathbf{c}^{\prime}(v)=0$ for each $W \in \mathcal{W}$. Recall that $(R, \mathcal{J})$ is a $\mathcal{W}$-partite Hamilton framework. It follows that $\mathcal{J}$ has no isolated vertices (thanks to having a perfect fractional matching) and is tightly connected. Moreover, if $r=1$, then our assumption implies that $\mathcal{J}$ also contains a closed tight walk of length coprime to $k$. These are precisely the conditions required by Proposition 7.5 (applied here with $\mathcal{W}$ and $\mathcal{J}$ in place of $\mathcal{U}$ and $\mathcal{H}$ ) to deduce 
that the 2-graph $(\hat{\mathcal{J}})[W]$ is connected for every $W \in \mathcal{W}$. Hence, by Proposition 7.6 (applied here with $\mathcal{W}, \mathcal{J}, \mathbf{c}^{\prime}$, 2t $\varrho$ n in place of $\mathcal{U}, \mathcal{H}, \mathbf{b}$ and $\left.s\right)$, there exists $\mathbf{w}_{5} \in \mathbb{Z}^{E(\mathcal{J})}$ with $A \mathbf{w}_{5}=\mathbf{c}^{\prime}$ and $\left\|\mathbf{w}_{5}\right\|_{\infty} \leqslant 2 k t^{3} \varrho n$. By the constant hierarchy $\left(\ll_{C}\right)$, we have $2 k t^{3} \varrho n \leqslant \pi n$ and the claim follows.

By the choice of $\mathbf{w}_{3}$ and $\left\|\mathbf{w}_{5}\right\|_{\infty} \leqslant \pi n$, we have that $\mathbf{w}_{3}+\mathbf{w}_{5} \geqslant 5 \pi n-\pi n \geqslant 4 \pi n \geqslant 0$. All together, it follows that $\mathbf{w}=\mathbf{w}_{2}+\mathbf{w}_{3}+\mathbf{w}_{4}+\mathbf{w}_{5}$ is such that $\mathbf{w} \in \mathbb{N}^{E(\mathcal{J})}$ has properties (i) to (iii), as desired.

Step 3: Defining $C$. Given the vector $\mathbf{w}$, we can easily setup the desired tight Hamilton cycle $\mathcal{C}$ in $\mathcal{J}^{*}$. We simply replace each $P_{e}$ with a tight path of length $k(\mathbf{w}(e)+1)$, which is possible since $\mathcal{J}^{*}$ is a $\mathcal{V}$-blow-up of $\mathcal{J}$. By the choice of $\mathbf{w}$, for every $e \in E(\mathcal{J})$ we have $\mathbf{w}(e) \geqslant 4 \pi n$, and for every $e \in E\left(\mathcal{J}^{\prime}\right)$ we have $\mathbf{w}(e) \geqslant 3 \alpha m$. This allows us to find vertexdisjoint subpaths $\left\{P_{e}\right\}_{e \in E(\mathcal{J})}$ of the required lengths in the subgraph of $\mathcal{J}^{*}$ induced by the clusters of every edge $e \in E(\mathcal{J})$.

We consider now the $(k-1)$ th power of a cycle $C$ given by $C=\partial_{2} \mathcal{C}$, and the $(k-1)$ th powers of a path $\partial_{2} P_{e} \subseteq C$. We now define the required $\left(\alpha, R^{\prime}\right)$-buffer in $C$ where, recall, $R^{\prime}=\partial_{2} \mathcal{J}^{\prime}$. Let $1 \leqslant i \leqslant r t$ and $V_{i} \in \mathcal{V}=V(\mathcal{J})$ be some cluster. Since $\mathcal{J}^{\prime}$ is a spanning subgraph of $\mathcal{J}$, we can fix an edge $K_{i} \in E\left(\mathcal{J}^{\prime}\right)$ such that $i \in V\left(K_{i}\right)$. By construction, we have ensured that $\mathbf{w}\left(K_{i}\right) \geqslant 3 \alpha \mathrm{m}$. This implies that $C$ contains a subpath $P_{K_{i}}$ of length at least $3 \alpha \mathrm{km}$ completely contained in the clusters of $K_{i}$. We have $\left|V\left(P_{K_{i}}\right) \cap X_{i}\right| \geqslant 3 \alpha m \geqslant 2 \alpha\left|X_{i}\right|$, where the latter inequality holds because $X$ is $(1+\varepsilon)$-balanced. Define $\tilde{X}_{i}$ from $V\left(\tilde{P}_{K_{i}}\right) \cap X_{i}$ by removing the first and last $2 k-2$ vertices according to the order of $P_{K_{i}}$. We claim that $\left\{\tilde{X}_{i}\right\}_{i=1}^{r t}$ is an $\left(\alpha, R^{\prime}\right)$-buffer for $C$. Indeed, $\tilde{X}_{i} \subseteq X_{i}$, and by construction, $\left|\tilde{X}_{i}\right| \geqslant 2 \alpha\left|X_{i}\right|-2 k+2 \geqslant \alpha\left|X_{i}\right|$. Now let $x \in \tilde{X}_{i}$ and $x y, y z \in E(C)$ such that $y \in X_{j}$ and $z \in X_{k}$. Since we removed the first and last $2 k-2$ vertices from $V\left(P_{K_{i}}\right) \cap X_{i}$, we actually have that $y, z \in V\left(P_{K_{i}}\right)$. This implies that $j, k \in K_{i}$. Since $K_{i} \in E\left(\mathcal{J}^{\prime}\right)$ and $R^{\prime}=\partial_{2} \mathcal{J}^{\prime}$, we have $i j, j k \in R^{\prime}$, as required.

\section{EMBEDDING GRAPHS OF SUBLINEAR BANDWIDTH}

In this section we give the proof of Theorem 3.5. As in the proof of Theorem 3.4, the proof has two central lemmas which prepare the graphs for the embedding; a 'Lemma for $G$ ' which deals with the host graph, and a 'Lemma for $\mathcal{H}$ ' which deals with the guest graph.

We begin by stating the corresponding 'Lemma for $G$ '. Note that the differences between the next lemma and the previous Lemma 8.2 (Lemma for $G$ - cycles) is that $\mathcal{J}^{\prime}$ is a tight Hamilton cycle here (instead of an arbitrary spanning bounded-degree subgraph as before), that we insist that $t \equiv 1 \bmod k$, and that we allow for zero-free frameworks.

Lemma 9.1 (Lemma for $G$ - bandwidth). Let $1 / n \ll 1 / t_{1} \ll 1 / t_{0} \ll \varepsilon \ll d \ll \mu, 1 / k$, and let $r \in\{1, k\}$. Let $\mathcal{U}$ be an $(n, r)$-sized partition, and let $(G, \mathcal{H})$ be a $\mu$-robust $\mathcal{U}$-partite Hamilton framework.

Then there are $t_{0} \leqslant t \leqslant t_{1}$, a spanning subgraph $G^{\prime} \subseteq G$, a U U-refining $(1+\varepsilon)$-balanced partition $\mathcal{V}$ of $G^{\prime}$ with $t$ clusters inside each clusters of $\mathcal{U}$, and a graph $R$ on tr vertices such that the following holds. Let $\mathcal{H}^{\prime}=\mathcal{H} \cap K_{k}\left(G^{\prime}\right)$, and let $\mathcal{J}$ be the $k$-graph induced by $\left(\mathcal{H}^{\prime}, \mathcal{V}\right)$. There also exists a tight Hamilton cycle $\mathcal{J}^{\prime} \subseteq \mathcal{J}$ so that, for $R^{\prime}=\partial_{2} \mathcal{J}^{\prime}$, we have:

(G1) $t \equiv 1 \bmod k$,

(G2) $e\left(G^{\prime}\right) \geqslant(1-\mu / 2) e(G)$,

(G3) $\left(G^{\prime}, \mathcal{V}\right)$ is an $(\varepsilon, d)$-regular $R$-partition, which is $(\varepsilon, d)$-super-regular on $R^{\prime}$ and

$(\mathrm{G} 4)(R, \mathcal{J})$ is a $(\mu / 4)$-robust $\mathcal{W}$-partite Hamilton framework and $(\mu / 4,2 / n, \mathcal{W})$-clustermatchable where $\mathcal{W}$ is the $(t, r)$-sized partition induced by $(\mathcal{V}, \mathcal{U})$.

Moreover, if $r=1$ and $(G, \mathcal{H})$ is $\mu$-robust aperiodic (resp. zero-free), then $(R, \mathcal{J})$ is $(\mu / 4)$ robust (resp. zero-free) as well.

The following is our 'Lemma for $\mathcal{H}$ '.

Lemma 9.2 (Lemma for $H$ ). Let $0<1 / n \ll \beta, 1 / z \ll \xi \ll \pi \ll \varepsilon, 1 / t, 1 / k$ and $\varepsilon \ll \alpha \ll$ $\mu, 1 / k, 1 / \Delta$, and $t \equiv 1 \bmod k$, and let $r \in\{1, k\}$. 
Let $\mathcal{U}$ be an $(n, r)$-sized partition, and let $G$ be a $\mathcal{U}$-partite graph. Let $\mathcal{V}$ be a $\mathcal{U}$-refining $(1+\varepsilon)$-balanced partition with $t$ clusters inside each cluster of $\mathcal{U}$, and let $R$ be a graph on $\operatorname{tr}$ vertices such that $(G, \mathcal{V})$ is an $R$-partition. Let $\mathcal{W}$ be the $(t, r)$-sized partition of $R$ induced by $(\mathcal{V}, \mathcal{U})$. Suppose that $(R, \mathcal{J})$ is a $\mu$-robust $\mathcal{W}$-partite Hamilton framework which is $(\mu, 2 / n, \mathcal{W})$-cluster-matchable. Let $\mathcal{J}^{\prime} \subseteq \mathcal{J}$ be a tight Hamilton cycle on vertex set $1,2, \ldots, t r$.

Let $H$ be a graph on $n$ vertices with $\Delta(H) \leqslant \Delta$ which admits an ordering $\sigma$ of its vertices with bandwidth at most $\beta$. Suppose one of the following is true:

(i) $r=1, H$ is $k$-colourable and $(R, \mathcal{J})$ is a $\mu$-robust aperiodic Hamilton framework,

(ii) $r=1, H$ admits a $(z, \beta)$-zero-free $(k+1)$-colouring under $\sigma$, and $(R, \mathcal{J})$ is a $\mu$-robust zero-free Hamilton framework,

(iii) $r=k$, and $H$ admits an equitable $k$-colouring.

Then there is a size-compatible (with $\mathcal{V}$ ) vertex partition $\mathcal{X}=\left\{X_{i}\right\}_{i=1}^{\text {tr }}$ of $V(H)$, and families of subsets $\tilde{X}=\left\{\tilde{X}_{i}\right\}_{i=1}^{t r}, Z=\left\{Z_{i}\right\}_{i=1}^{t r}$, such that

(H1) $(H, X)$ is an R-partition,

(H2) $\tilde{X}$ is an $\left(\alpha, R^{\prime}\right)$-buffer for $(H, X)$ where $R^{\prime}=\partial_{2} \mathcal{J}^{\prime}$,

(H3) for each $1 \leqslant i \leqslant t, Z_{i} \subseteq X_{i} \backslash \tilde{X}_{i},\left|Z_{i}\right| \geqslant \pi\left|X_{i}\right|$, and for each $v \in Z_{i}, N_{H}(v) \subseteq$ $X_{i-1} \cup \cdots \cup X_{i-k+1}$, with indices understood modulo $t$, and

(H4) $\left|X_{i}\right|=\left|V_{i}\right| \pm \xi n$ for each $i \in V(R)$.

Assuming the validity of the previous two lemmas, we now give the proof of Theorem 3.5.

Proof of Theorem 3.5. We establish the following constant hierarchy

$$
1 / n \ll \beta, 1 / z \ll \xi \ll \pi \ll 1 / t_{1} \ll 1 / t_{0} \ll \varepsilon \ll d, \alpha \ll \mu, 1 / k .
$$

Given $k, \Delta \geqslant 1$ and $\mu>0$, choose $d>0$ that satisfies Lemma 9.1. Let $\zeta=1 / 2$. Then select $\varepsilon_{0}, \varrho>0$, so that Lemma 6.7 (Blow-Up Lemma) can be applied with $\Delta=\max \{\Delta, 2(k-1)\}$, $\Delta_{R^{\prime}}=2(k-1), \varrho=\xi, \kappa=2, \alpha, \zeta$ and $d$. Next, pick $0<\beta, \xi, \pi, \varepsilon \leqslant \varepsilon_{0}$ and $t_{0}, t_{1}, z \in \mathbb{N}$ such that the conditions of Lemmas 9.1 and 9.2 (where we apply the latter with $\mu / 4$ instead of $\mu$ ) are satisfied with these choices. We obtain $n_{0}$ from Lemma 6.7 with input $\varepsilon$ and $t_{1}$. Finally, choose an integer $n \geqslant n_{0}$, such that the conditions of Lemmas 9.1 and 9.2 are satisfied (together with the above choices).

The proofs of items (i) to (iii) are very similar and follow the same overall structure. We will prove item (ii) with full details, and outline the necessary changes to prove items (i) and (iii) later.

To prove item (ii), assume that $(G, \mathcal{H})$ is a $\mu$-robust aperiodic Hamilton framework. Note that this implies $r=1$ and $\mathcal{U}$ is a trivial partition. Let $H$ be an arbitrary $k$-chromatic graph on $n$ vertices with $\Delta(H) \leqslant \Delta$ and bandwidth at most $\beta n$. To prove item (ii), we have to show that $H \subseteq G$.

Step 1: Applying the Lemma for $G$. By Lemma 9.1 there are $t_{0} \leqslant t \leqslant t_{1}$, a spanning subgraph $G^{\prime} \subseteq G$, a $(1+\varepsilon)$-balanced partition $\mathcal{V}=\left\{V_{i}\right\}_{i=1}^{t}$ of $V\left(G^{\prime}\right)$, and a graph $R$ on $t$ vertices such that following is true. Let $\mathcal{H}^{\prime}=\mathcal{H} \cap K_{k}\left(G^{\prime}\right)$, and let $\mathcal{J}$ be the $k$-graph induced by $\left(\mathcal{H}^{\prime}, \mathcal{V}\right)$. Then there exists a tight Hamilton cycle $\mathcal{J}^{\prime} \subseteq \mathcal{J}$ so that, for $R^{\prime}=\partial_{2} \mathcal{J}^{\prime}$,

(G1) $t \equiv 1 \bmod k$,

(G2) $e\left(G^{\prime}\right) \geqslant(1-\mu / 2) e(G)$,

(G3) $\left(G^{\prime}, \mathcal{V}\right)$ is an $(\varepsilon, d)$-regular $R$-partition, which is $(\varepsilon, d)$-super-regular on $R^{\prime}$, and

(G4) $(R, \mathcal{J})$ is a $(\mu / 4)$-robust $\mathcal{W}$-partite Hamilton framework which is $(\mu / 4,2 / n, \mathcal{W})$ cluster-matchable where $\mathcal{W}$ is the $(t, 1)$-sized partition induced by $(\mathcal{V}, \mathcal{U})$.

Since $r=1$ and $(G, \mathcal{H})$ is $\mu$-robust aperiodic, we are also ensured (by the last part of Lemma 9.1) that $(R, \mathcal{J})$ is $(\mu / 4)$-robust aperiodic. Since $\mathcal{U}$ is the trivial partition on $V\left(G^{\prime}\right)$, then $\mathcal{W}$ also corresponds to a trivial partition of the clusters of $\mathcal{V}$. From this we deduce

$\left(\mathrm{G} 4^{\prime}\right)(R, \mathcal{J})$ is a $(\mu / 4)$-robust aperiodic Hamilton framework which is $(\mu / 4,2 / n)$-clustermatchable. 
Without loss of generality (by relabelling clusters, if necessary) we assume the tight Hamilton cycle $\mathcal{J}^{\prime}$ has cyclic order following the indices $1,2, \ldots, t$.

Step 2: Applying the Lemma for $H$. Next, we apply Lemma 9.2 (with $\mu / 4$ in place of $\mu$ ) to obtain a size-compatible (with $\mathcal{V}$ ) vertex partition $\mathcal{X}=\left\{X_{i}\right\}_{i=1}^{t}$ of $V(\mathcal{H})$ and two families of subsets, $\tilde{X}=\left\{\tilde{X}_{i}\right\}_{i=1}^{t}$ and $Z=\left\{Z_{i}\right\}_{i=1}^{t}$ of $V(\mathcal{H})$, such that

(H1) $(H, X)$ is an $R$-partition,

(H2) $\tilde{X}$ is an $\left(\alpha, R^{\prime}\right)$-buffer for $(H, X)$,

(H3) for each $1 \leqslant i \leqslant t, Z_{i} \subseteq X_{i} \backslash \tilde{X}_{i}$ and $\left|Z_{i}\right| \geqslant \pi\left|X_{i}\right|$, and for each $v \in Z_{i}, N_{H}(v) \subseteq$ $X_{i-1} \cup \cdots \cup X_{i-k+1}$, with indices understood modulo $t$, and

(H4) $\left|X_{i}\right|=\left|V_{i}\right| \pm \xi n$ for each $i \in V(R)$.

Step 3: Adjusting the partition of $G$. Consider integers $m_{1}, \ldots, m_{t}$ such that $\left|X_{i}\right|=\left|V_{i}\right|+m_{i}$ for $1 \leqslant i \leqslant t$. Note that item (H4) implies that $-\xi n \leqslant m_{i} \leqslant \xi n$, and we also have $\sum_{i=1}^{t} m_{i}=0$. In particular, we have $\lfloor\sqrt{\xi} n\rfloor \geqslant \sum_{i=1}^{t}\left|m_{i}\right|$ since $\xi \ll 1 / t$. Let us now select $U_{i} \subseteq V_{i}$ for every $1 \leqslant i \leqslant t$, as follows. Let $1 \leqslant j \leqslant k$ such that $i \equiv j k \bmod t$, which exists and is unique since $t$ is coprime to $k$ by item (G1). Then choose $U_{j k} \subseteq V_{j k}$ such that $\left|U_{j k}\right|=\lfloor\sqrt{\xi} n\rfloor-\sum_{\ell=1}^{j} m_{\ell k}$ for each $1 \leqslant j \leqslant t$, where the indices are evaluated modulo $t$. Define $\mathcal{V}^{\prime}=\left\{V_{i}^{\prime}\right\}_{i=1}^{t}$ by setting $V_{j k}^{\prime}=V_{j k} \backslash U_{j k}$ for every $1 \leqslant j \leqslant t$. We remark that $\left(G^{\prime}, \mathcal{V}^{\prime}\right)$ is a $(2 \varepsilon, d)$-regular $R$-partition, which is $(2 \varepsilon, d)$-super-regular on $R^{\prime}$ by Proposition 6.1 (Robust regular and super-regular pairs). Moreover, we have for all $1 \leqslant j \leqslant t$,

$$
\begin{aligned}
\left|V_{j k}^{\prime} \cup U_{(j-1) k}\right| & =\left|V_{j k} \backslash U_{j k}\right|+\left|U_{(j-1) k}\right| \\
& =\left|V_{j k}\right|-\left(\lfloor\sqrt{\xi} n\rfloor-\sum_{\ell=1}^{j} m_{\ell k}\right)+\left(\lfloor\sqrt{\xi} n\rfloor-\sum_{\ell=1}^{j-1} m_{\ell k}\right) \\
& =\left|V_{j k}\right|+m_{j k}=\left|X_{j k}\right| .
\end{aligned}
$$

Step 4: Applying the Blow-Up Lemma. To finish, we define a subgraph $H^{\prime} \subseteq H$, a family of image restrictions $\mathcal{J}=\left\{I_{x}\right\}_{x \in V(\mathcal{H})}$, and a family of restricting vertices $\mathcal{J}=\left\{J_{x}\right\}_{x \in V(\mathcal{H})}$. To do this, consider the sets $Z_{i} \subseteq X_{i}$ given by item (H3). We will select a set $Y_{i} \subseteq Z_{i}$ of size $\left|U_{i-k}\right|$ such that every two elements of $Y=\bigcup_{i} Y_{i}$ have distance at least four in $\mathcal{H}$ (meaning that their neighbourhoods do not intersect). Such a set $Y_{i}$ can be found greedily. Indeed, by item (H3) we have $\left|Z_{i}\right| \geqslant \pi\left|X_{i}\right|$. For each $y \in Z_{i}$ there are at most $\Delta^{3}$ vertices in $z \in Z_{i}$ whose neighbourhoods are not disjoint. We can then pick available vertices greedily, each new choice will inhabilitate at most $\Delta^{3}$ new vertices. This implies we can select a set inside $Z_{i}$ of size at least $\pi\left|X_{i}\right| / \Delta^{3}$, and vertices in this set are pairwise at distance at least four in $G$. But such a set has size at least $\left|U_{i-k}\right|$, since $\left|U_{i-k}\right| \leqslant \xi^{1 / 2} n$ and $\xi \ll 1 / t, \pi, 1 / \Delta$ per the choice of the constant hierarchy.

Next, define $U=\bigcup_{i=1}^{t} U_{i}$ and choose an arbitrary bijection $\psi^{\prime}: Y \rightarrow U$ that maps $Y_{i}$ to $U_{i-k}$ for all $1 \leqslant i \leqslant t$. This exists, because $\left|Y_{i}\right|=\left|U_{i-k}\right|$ for all $1 \leqslant i \leqslant t$. Now we define the necessary restriction pairs to apply the Blow-Up Lemma, for all vertices in $V(H) \backslash Y$. For vertices $x \in V(H) \backslash\left(\bigcup_{y \in Y} N_{H}(y) \cup Y\right.$ ), we simply set $J_{x}=\emptyset$ and $I_{x}=V_{j}^{\prime}$ (where $x \in X_{j}$ ). It is left to consider the vertices in $\bigcup_{y \in Y} N_{H}(y)$. Given some $y \in Y_{i}$ and $x \in N_{H}(y)$, we will define $J_{x}$ and $I_{x}$ as follows. Since $Y_{i} \subseteq Z_{i}$, by item (H3) we have $x \in X_{i-1} \cup \cdots \cup X_{i-k+1}$. Let $i-k+1 \leqslant j \leqslant i-1$ be such that $x \in X_{j}$. Now let $u=\psi^{\prime}(y) \in U_{i-k}$. We define $J_{x}=\{u\}$ and $I_{x}=N_{G^{\prime}}(u) \cap V_{j}^{\prime}$. We check that this choice ensures $\left|I_{x}\right|$ is sufficiently large: since $\mathcal{J}^{\prime}$ is a tight Hamiltonian cycle, the edge $i j$ exists in $R^{\prime}=\partial_{2} \mathcal{J}^{\prime}$. Then, since $\left(G^{\prime}, \mathcal{V}^{\prime}\right)$ is $(2 \varepsilon, d)$-regular on $R^{\prime}$, we have that $\left|I_{x}\right|=\left|N_{G^{\prime}}(u) \cap V_{j}^{\prime}\right| \geqslant(d-2 \varepsilon)\left|V_{j}^{\prime}\right| \geqslant \zeta d\left|V_{j}^{\prime}\right|$, by the choice of $\zeta$ at the beginning of the proof. Next, notice that each vertex in $Y_{i}$ can restrict the image of at most $\Delta$ vertices of $V_{i-k+1}^{\prime}, \ldots, V_{i-1}^{\prime}$. Therefore, the number of image-restricted vertices in each $V_{i}^{\prime}$ is at most $\Delta \sum_{i+1 \leqslant j \leqslant i+k-1}\left|Y_{j}\right| \leqslant \Delta k \xi^{1 / 2} n \leqslant \varrho\left|V_{i}^{\prime}\right|$. It follows that $\mathcal{J}$ and J form a $(\varrho, \zeta, \Delta, \Delta)$-restriction pair. 
Finally, we apply the Blow-Up Lemma (Lemma 6.7) with $G=G^{\prime}-U, H=H^{\prime}, \Delta=$ $\max \{\Delta, 2(k-1)\}, \Delta_{R^{\prime}}=2(k-1)$ and $1+\varepsilon \leqslant 2=\kappa$ to obtain an embedding $\psi: V\left(H^{\prime}\right) \rightarrow$ $V(G)$ such that $\psi(x) \in I_{x}$ for each $x \in V(H)$. It follows that $G$ contains a copy of $H$. Since $H$ was arbitrary, this shows that $G$ is $(\beta, \Delta, k)$-Hamiltonian. This concludes the proof of part (ii) of 3.5 .

Step 5: The zero-free case. We show part (iii) of Theorem 3.5. For this, our assumption is that $r=1$ and $(G, \mathcal{H})$ is a $k$-uniform $\mu$-robust zero-free Hamilton framework. For an arbitrary graph $H$ on $n$ vertices with $\Delta(H) \leqslant \Delta$ which admits a $(z, \beta)$-zero-free $(k+1)$-colouring, we need to show $H \subseteq G$.

The proof follows the same steps as before. Step 1 is virtually the same, but now since $(G, \mathcal{H})$ is zero-free and not just aperiodic, the application of Lemma 9.1 yields that, instead of item $\left(\mathrm{G} 4^{\prime}\right)$, we get that $(R, \mathcal{J})$ is a $(\mu / 4)$-robust zero-free Hamilton framework and $(\mu / 4,2 / n)$ cluster-matchable. In Step 2, this extra property allows us to apply Lemma 9.2 with $H$ as an input. The remainder of the proof is exactly the same.

Step 6: The partite case. To show part (i) of 3.5, again we essentially follow the same steps. Now the assumption is that $r=k$ and $(G, \mathcal{H})$ is a $k$-uniform $\mu$-robust $\mathcal{U}$-partite Hamilton framework. To show that $G$ is U-partite $(\beta, \Delta, k)$-Hamiltonian, we select an arbitrary graph $H$ on $n k$ vertices with an equitable $k$-colouring, $\Delta(H) \leqslant \Delta$ and bandwidth at most $\beta k n$; and we need to show $H \subseteq G$.

In this situation, the application of Lemma 9.1 in Step 1 yields that $(R, \mathcal{J})$ is a $(\mu / 4)$ robust $\mathcal{W}$-partite Hamilton framework and $(\mu / 4,2 / n, \mathcal{W})$-cluster matchable where $\mathcal{W}$ is the $(t, r)$-sized partition induced by $(\mathcal{V}, \mathcal{U})$. This allows us to apply Lemma for $H$ in Step 2 with $H$ as an input, and obtain a vertex partition $X$ of $V(H)$ and families $\tilde{X}$, z satisfying items $(\mathrm{H} 1)$ to $(\mathrm{H} 4)$. Let $\mathcal{W}$ be the $(t, r)$-sized partition induced by $(\mathcal{V}, \mathcal{U})$. Since $\mathcal{X}$ is sizecompatible with $\mathcal{V}$, and $\mathcal{V}$ refines $\mathcal{U}$, which is $(n, r)$-sized, we have $\sum_{i \in W}\left|X_{i}\right|=n$ for each $W \in \mathcal{W}$.

In Step 3, we proceed similarly but now adjusting the allocation separately in each of the $k$ clusters of $\mathcal{U}$. With more detail, recall that the Hamilton cycle $\mathcal{J}^{\prime} \subseteq \mathcal{J}$ is given by $1, \ldots, t r$ on $V(R)$. Define $m_{i}=\left|X_{i}\right|-\left|V_{i}\right|$ for all $1 \leqslant i \leqslant t r$. Again, we have $-\xi n \leqslant m_{i} \leqslant \xi n$. Note that, for any $W \in \mathcal{W}$, there exists $1 \leqslant q \leqslant k$, such that the clusters of $\mathcal{V}$ contained in $\mathcal{W}$ correspond exactly to $\left\{V_{q+i k}\right\}_{i=0}^{t-1}$. Now fix any $W \in \mathcal{W}$, we have $\sum_{i \in W} m_{i}=0$. This allows us to define $U_{i}, V_{i}^{\prime}$ as before, again by considering steps of $k$ clusters, and the rest of the proof follows as before.

9.1. Proof of the Lemma for $G$ - bandwidth. The proof of Lemma 9.1 is almost identical to the one of Lemma 8.2, so we will only sketch the main differences.

Proof of Lemma 9.1 (sketch). We begin the proof as the one of Lemma 8.2 by choosing the constants. The only difference is that we choose $\gamma, \pi, \eta, t$ so that Theorem 8.1 (Distributed Hamiltonicity) can be applied with $\gamma$ in place of $\mu$, which will replace Proposition 7.1 (Distributed matching).

We then proceed as before with Step 1 and find a balanced regular $R$-partition $\left(G^{\prime}, \mathcal{V}\right)$ together with a collection $\mathcal{W}$ of subgraphs of $\mathcal{J}$ satisfying properties (R1) to (R6). In addition, whenever we apply Lemma 7.11 we insist that $t \equiv 1 \bmod k$, as required here. We also note that if $(G, \mathcal{H})$ is $\mu$-robust zero-free (and not just aperiodic) then we can replace (R3) by

(R3') $(R, J)$ is a $(\mu / 4)$-robust zero-free Hamilton framework and is also $(\mu / 4,2 / n)$-clustermatchable.

Then, in Step 2, we apply Theorem 8.1 (Distributed Hamiltonicity) (with $\gamma$ playing the role of $\mu$ ) to $\mathcal{J}$. It follows that $R$ has as a subgraph the $(k-1)$ th power of a Hamilton cycle $R^{\prime}$ such that $\mathcal{J}^{\prime}:=K_{k}\left(R^{\prime}\right)$ contains at least $\pi t$ edges of each element of $\mathcal{W}$. The remaining parts of the proof (Steps 3 and 4 ) follow in exactly the same way as before. 


\section{Proof of the Lemma for $H$}

In this section, we show Lemma 9.2. We first sketch of the argument and then proceed to show the main steps in Sections 10.3 to 10.5 followed by the proof itself in Section 10.6.

10.1. Proof overview. We proceed in a series of steps to prove Lemma 9.2. Assume we are given a $(k+1)$-colouring of $H$ with a low-bandwidth ordering, and a zero-free framework $R$. First we will partition $V(H)$ into runs of consecutive vertices $H_{1}, \ldots, H_{r}$, where in each $H_{i}$ few vertices at the beginning are coloured using the colour zero, and the remaining vertices are $k$-coloured. For each $i$ we will set $H_{i}^{-}, H_{i}^{+} \subseteq H_{i}$ as short initial and final intervals of $H_{i}$, where in particular $H_{i}^{-}$contains all zero-coloured vertices of $H_{i}$. The required allocation for $H$ will consist in two main parts: in an initial pass we will allocate the bulk of the vertices, which lie in $\bigcup_{i} H_{i}-\left(H_{i}^{-} \cup H_{i}^{+}\right)$. They will be allocated using a semi-randomised algorithm, which will be described with more detail next. In the second part, we will complete the allocation by including the remaining vertices in $\bigcup_{i} H_{i}^{-} \cup H_{i}^{+}$. This last part will be done by allocating these vertices 'by hand' into $R$, making sure the runs are connected in an appropriate way and we embed the $(k+1)$-colourable part of $H_{i}$ in a $(k+1)$-clique of $R$ (which exists if $R$ is a zero-free framework).

Now we describe the first part of the embedding sketched before. To describe the allocation assume the graph we need to allocate is $H$, a $k$-colourable graph on $n$ vertices (if $H$ itself is $k$-colourable then this is exactly what we do, otherwise we use this method considering $\bigcup_{i} H_{i}-\left(H_{i}^{-} \cup H_{i}^{+}\right)$, which is $k$-colourable). There are three steps in our embedding. Initially, we will show $H$ can be allocated into the blow-up of a long $(k-1)$ th power of path $P$. This is done deterministically, by simply scanning the vertices using the bandwidth ordering and allocating the vertices into the corresponding coloured vertices of $P$. However, since the $k$-colouring of $H$ can have colour classes of wildly different sizes, this does not quite give the balanced allocation we want.

In a second step, we correct this by embedding the clusters of $(k-1)$ th power of a path $P$ in a $(k-1)$ th power of a cycle $C$ by simulating a random walk of $P$ over the vertices of $C$; which in turn gives an allocation of $H$ into $C$ (Lemma 10.3). We analyse this random allocation using a Markov chain, and under mild conditions we will be able to show this gives a very balanced allocation of $H$ into $C$, with clusters of almost the same size, say up to error $\xi n /|C|$ each. At this point we could try to use such an allocation using $C=R^{\prime}$ where $R^{\prime}$ is the given $(k-1)$ th power of a Hamilton cycle in $R$. However, this is still not quite what we need. This is because in the statement of Lemma 9.2 we require to find an allocation of $H$ into $t$ clusters whose size matches the sizes of $\mathcal{V}$, which is a $(1+\varepsilon)$-balanced allocation instead of a perfectly balanced allocation. Since $\xi \ll \varepsilon$, our allocation up to this point does not necessarily approximate the cluster sizes of $\mathcal{V}$ in a good way.

In a final step, we overcome this last obstacle by fixing some large $\ell$ (depending on $\varepsilon, t$ only), finding a homomorphism of a power of a cycle $C^{\prime}$ on $\ell$ vertices into $R$ such that each vertex $i \in V(R)$ is used a number of times proportional to $\left|V_{i}\right| / n$ (Lemma 10.7). Thus, finally our desired allocation is found by embedding first $H$ into $C^{\prime}$, and then $C^{\prime}$ into $R$. Since the first embedding is almost balanced, and the second one accounts for the varying sizes of the clusters of $R$, the final allocation will satisfy the required size conditions of the partition $\mathcal{V}$.

10.2. Splitting into intervals. The first step is to allocate our guest graph $H$ into a (modified) power of a path, which allows us to distinguish between the $k$ - and $(k+1)$ colourable cases. If the given $k$-colouring is also equitable, this will also be captured by the allocation.

Given $k, t$ integers, let $P_{k, t}$ be the $(k-1)$ th power of a path on $t$ vertices, we will consider $V\left(P_{k, t}\right)=\left\{p_{1}, \ldots, p_{t}\right\}$ in the natural ordering. Let $P_{k, t}^{+}$be the graph obtained from $P_{k, t}$ by adding an extra vertex $*$, which is joined to all vertices of $V\left(P_{k, t}\right)$.

Given $a, b>0$, if $\phi: V(G) \rightarrow V(H)$ satisfies $\left|\phi^{-1}(v)\right| \leqslant a$ for all $v \in V(H)$, we say $\phi$ is at most $a$-to-one. If it satisfies $a-b \leqslant\left|\phi^{-1}(v)\right| \leqslant a+b$, we say $\phi$ is $(a \pm b)$-to-one. By $k \mathbb{N}$, we will mean the set of all multiples of $k$. 
Lemma 10.1 (Allocating $H$ to a power of a path). Let $1 / n \ll \beta, 1 / k$ and $q=\left\lceil\beta^{-1}\right\rceil+k-1$. Let $H$ be a graph on $n$ vertices with an ordering of bandwidth at most $\beta n$, and suppose $H$ has $a(z, \beta)$-zero-free $(k+1)$-colouring $\chi$ with respect to the given ordering. For all $0 \leqslant j \leqslant k$, let $H_{j}=\chi^{-1}(\{j\})$ denote the $j$-coloured vertices of $H$. Let $p_{1}, \ldots, p_{q}$ be the vertex set of $P=P_{k, q}$, and for each $1 \leqslant j \leqslant k$ let $P_{j}=\left\{p_{i}: i \in j+k \mathbb{N}\right\}$. Then there exists a homomorphism $\phi: V(H) \rightarrow V\left(P_{k, q}^{+}\right)$such that

(P1) $\phi^{-1}(\{*\})=H_{0}$,

(P2) for each $1 \leqslant j \leqslant k, \phi^{-1}\left(P_{j}\right)=H_{j}$,

(P3) $\phi$ restricted to $V(H) \backslash H_{0}$ is an at most $(k\lceil\beta n\rceil)$-to-one homomorphism to $P_{k, q}$, and

(P4) there exist $s \geqslant 0$ and indices $1 \leqslant i_{1} \leqslant i_{2} \leqslant \cdots \leqslant i_{s} \leqslant q$ such that

(a) $i_{j+1}-i_{j} \geqslant z$ for all $1 \leqslant j<s$,

(b) for every $v \in V(H)$ adjacent to a vertex in $H_{0}$, there exists $1 \leqslant j<s$ such that $\phi(v) \in\left\{p_{i_{j}-1}, \ldots, p_{i_{j}+k}\right\}$.

Proof. Initially, set $\phi(x)=*$ for all $x \in H_{0}$. It thus remains only to allocate the vertices of $H_{*}=V(H) \backslash H_{0}$ along $P_{k, q}$ to complete the definition of $\phi$.

Consider the given ordering $v_{1}, \ldots, v_{n}$ of $V(H)$, with satisfies the corresponding bandwidth and zero-free colouring properties. Partition $V(H)$ along its consecutive $\beta$-blocks. More precisely, we divide $V(H)$ into $q-k+1$ intervals $X_{1}, \ldots, X_{q-k+1}$ of consecutive vertices as follows: initially, greedily select intervals of consecutive vertices of size exactly $\lceil\beta n\rceil$, until this is no longer possible. Then, choose a single interval of size less than $\lceil\beta n\rceil$ covering the remaining of the vertices, and finalise by declaring all of the next intervals to be empty.

For each $1 \leqslant i \leqslant q-k+1$ and $0 \leqslant j \leqslant k$, let $X_{i}^{j}=\chi^{-1}(j) \cap X_{i}$, that is, the vertices in $X_{i}$ which were $j$-coloured under $\chi$. Note that $H_{*}=\bigcup_{1 \leqslant i \leqslant q-k+1,1 \leqslant j \leqslant k} X_{j}^{i}$. Certainly, $\left|X_{i}^{j}\right| \leqslant\left|X_{i}\right| \leqslant\lceil\beta n\rceil$ for all $i, j$. Because of the colouring and the bandwidth condition, a vertex in $X_{i}^{j}$ can only have neighbours in $X_{i^{\prime}}^{j^{\prime}}$ if $\left|i-i^{\prime}\right| \leqslant 1$ and $j \neq j^{\prime}$.

The idea is to allocate the vertices of $H_{*}$ in $P$ in a way such that the following two properties are satisfied:

(i) $p_{1}$ only receives 1 -coloured vertices, $p_{2}$ only receives 2 -coloured vertices, and so on, and this pattern is repeated cyclically; and

(ii) the vertices of $X_{i} \backslash H_{0}$ are allocated only to $\left\{p_{i}, p_{i+1}, \ldots, p_{i+k-1}\right\} \subseteq V(P)$, for each $1 \leqslant i \leqslant q-k+1$.

By the previous observations, this will yield a valid homomorphism. Formally, this idea is accomplished by the function $\phi: H_{*} \rightarrow P$ which sends all of $X_{i}^{j}$ to $p_{k\lfloor(i-j+k-1) / k\rfloor+j}$, for all $1 \leqslant i \leqslant q-k+1$ and $1 \leqslant j \leqslant k$. The previous discussion ensures $\phi$ is graph homomorphism when $H$ is restricted to $H_{*}$. By construction, for each $v \in V(P)$ we have $\left|\phi^{-1}(v)\right| \leqslant k\lceil\beta n\rceil$, as required.

To verify the last condition, we examine the position of the zero-coloured vertices in the $\beta$ blocks. Let $Z=\left\{i_{1}, \ldots, i_{s}\right\} \subseteq\{1, \ldots, q-k+1\}$ be the set of indices $i$ such that $X_{i}^{0} \neq \emptyset$. Since $\chi$ is a $(z, \beta)$-zero-free colouring, we obtain $i_{j+1}-i_{j} \geqslant z$ for all $1 \leqslant j<s$. Now, let $x \in H_{0}$ and $1 \leqslant j \leqslant s$ such that $x \in X_{i_{j}}$. If $v \in V(H)$ is adjacent to $x$, then $v \in\left(X_{i_{j}-1} \cup X_{i_{j}} \cup X_{i_{j}+1}\right) \backslash H_{0}$. By definition of $\phi$, it holds that $\phi\left(X_{i} \backslash H_{0}\right) \subseteq\left\{p_{i}, \ldots, p_{i+k-1}\right\}$ for all $1 \leqslant i \leqslant q$. We deduce that $\phi(v) \in \phi\left(X_{i_{j}-1} \backslash H_{0}\right) \cup \phi\left(X_{i_{j}-1} \backslash H_{0}\right) \cup \phi\left(X_{i_{j}+1} \backslash H_{0}\right) \subseteq\left\{p_{i_{j}-1}, \ldots, p_{i_{j}+k}\right\}$, as required.

10.3. Markov chains and concentration. We will work with finite Markov chains, which are random processes $\left\{X_{i}\right\}_{i \geqslant 1}$ taking values in a state space $\mathcal{X}$. The trajectories of the random process are determined by a transition matrix $P$, whose columns and rows are indexed by $\mathcal{X}$. A Markov chain is irreducible if for all $i, j \in \mathcal{X}$ there exists $t>0$ such that $P_{i j}^{t}>0$ where $P^{t}$ is the $t$ th power of the matrix $P$ and $P_{i j}^{t}$ is the entry in the $i$ th row and $j$ th column. The period $d_{i}$ of an state $i \in \mathcal{X}$ is the greatest common divisor of the numbers in $\left\{t>0: P_{i i}^{t}>0\right\}$, and a Markov chain is aperiodic if $d_{i}=1$ for all $i \in \mathcal{X}$. A probability distribution $\pi$ on $\mathcal{X}$ (understood as a column vector) is stationary for the Markov chain if 
$\pi=\pi P$. A basic fact from Markov chain theory states that finite and irreducible Markov chains admit a unique stationary distribution.

We recall that, for finite Markov chains, irreducibility can also be expressed in a more combinatorial way in terms of strong connectivity of directed graphs. Let $D=D(P, \mathcal{X})$ be the directed graph on $\mathcal{X}$ where $a \rightarrow b$ whenever $P_{a b}>0$. Then the Markov chain $\left\{X_{i}\right\}_{i \geqslant 1}$ is irreducible if and only if $D$ is strongly connected, meaning that there exists a directed walk between any two arbitrary vertices of $D$. Similarly, an irreducible and finite Markov chain will be aperiodic if $D$ contains two directed closed walks whose lengths are coprime.

The following result is a concentration inequality for Markov-dependent random variables. The first inequality of this kind was proven by Gillman [28], although many variations exist (see, e.g., Section 3.5 in [21]). We will use a recent Hoeffding-type inequality for Markov chains, as proven by Fan, Jiang and Sun [24] and independently by Rao [65].

We use the following notation: given a function $f: \mathcal{X} \rightarrow \mathbb{R}$ and a probability distribution $\pi$ over $\mathcal{X}$, we write $\mathbf{E}_{\pi}\left[f_{i}\right]=\sum_{x \in X} \pi(x) f_{i}(x)$.

Theorem 10.2 ([24, Theorem 2.1]). Let $\left\{X_{i}\right\}_{i \geqslant 1}$ be a finite, irreducible and aperiodic Markov chain with state space $\mathcal{X}$ and transition matrix $P$. There exists $C>0$, depending on $\mathcal{X}$ and $P$ only, such that the following holds.

Suppose $X_{1}$ is distributed according to the stationary distribution $\pi$ of the chain. Let $\left\{f_{i}\right\}_{i \geqslant 1}$ be functions $f_{i}: \mathcal{X} \rightarrow\left[a_{i}, b_{i}\right]$. Then, for every $\varepsilon>0$,

$$
\operatorname{Pr}\left[\left|\sum_{i=1}^{r} f_{i}\left(X_{i}\right)-\sum_{i=1}^{r} \mathbf{E}_{\pi}\left[f_{i}\right]\right|>\varepsilon\right] \leqslant 2 \exp \left(-\frac{C \varepsilon^{2}}{2 \sum_{i=1}^{r}\left(b_{i}-a_{i}\right)^{2} / 4}\right) .
$$

We remark that the original statement of [24, Theorem 2.1] applies to the larger class of (possibly infinite) Markov chains with positive absolute spectral gap. We state it only for the particular case we need, which is that of finite, irreducible and aperiodic Markov chains, it is well-known that these properties imply the Markov chain has a non-trivial spectral gap.

10.4. Randomised allocation. In this subsection we will construct an allocation of a guest graph $H$ into a power of a cycle, focusing on the case where $H$ is $k$-colourable. We account for the aperiodic case (where $r=1$ ) and the partite case (where $r=k$ ) here as well. In the following, we denote by $C_{k, t}$ the $k$ th power of a Hamilton cycle on $t$ vertices.

Lemma 10.3 (Randomised allocation for $H$ ). Let $1 / n \ll \beta, 1 / q \ll \xi, 1 / t, 1 / k$ and such that $t, k$ are coprime, and $r \in\{1, k\}$. Let $P_{k, q}=\left(p_{1}, \ldots, p_{q}\right), H$ be a graph on nr vertices and $\phi_{1}: V(H) \rightarrow V\left(P_{k, q}\right)$ a homomorphism which is at most $(k\lceil\beta r n\rceil)$-to-one. Suppose in addition that if $r=k$, then $\left|\phi^{-1}\left(P_{j}\right)\right|=n$ where $P_{j}=\left\{p_{i}: i \in j+k \mathbb{N}\right\}$.

Then there exists a homomorphism $\phi: V(H) \rightarrow V\left(C_{k, r t}\right)$ which is $\left((1 \pm \xi) \frac{n}{t}\right)$-to-one.

As sketched before, we will prove Lemma 10.3 by embedding $P_{k, q}$ into $C_{k, r t}$ appropriately and combining this with the given $\phi_{1}$. We will describe a process which finds a homomorphism from $P_{k, q}$ into $C_{k, r t}$ using a randomised algorithm. To explain the idea, suppose that vertices $p_{1}, \ldots, p_{s}$ in $P_{k, q}$ have been embedded and $p_{s-k+2}, \ldots, p_{s}$ was mapped on the vertex $c_{i-k+2}, \ldots, c_{i}$ of $C_{k, r t}$. If we were simulating a simple random walk in the cycle, we would then like to map $p_{s+1}$ uniformly on $c_{i+1}$ or $c_{i-1}$, where the index computations are modulo $r t$. When $k=2$, this is fine. But for $k>2$, we have to avoid the $k-1$ trailing vertices of $P_{k, q}$ in order to maintain the homomorphism property. We achieve this by 'looping around' the last $k-1$ vertices. This means we embed $p_{s+1}$ instead in a cluster chosen uniformly at random between $c_{i+1}$ or $c_{i-(k-1)}$. In the latter case, we afterwards map $p_{s+2}, \ldots, p_{s+k-2}$ deterministically to $c_{i-(k-2)}, \ldots, c_{i-1}$, before doing the next random choice, and so on. We formalise this construction as follows.

The above outlined algorithm can be modelled with a Markov chain $X=\left\{X_{i}\right\}_{i \geqslant 1}$. The state space of $X$ is $\mathcal{X}_{k, r t}=\{1, \ldots, r t\} \times\{1, \ldots, k-1\}$. The transition probabilities $P\left((a, b),\left(a^{\prime}, b^{\prime}\right)\right)$ for all $(a, b),\left(a^{\prime}, b^{\prime}\right) \in\{1, \ldots, t\} \times\{1, \ldots, k-1\}$ are given by

$$
P((a, 1),(a+1,1))=1 / 2,
$$




$$
\begin{aligned}
P((a, 1),(a, 2)) & =1 / 2, \\
P((a, b),(a, b+1)) & =1 \\
P((a, k-1),(a-1,1)) & =1,
\end{aligned}
$$$$
\text { if } j \in\{2, \ldots, k-2\} \text {, }
$$

and 0 otherwise (sums in the indices are always understood modulo $r t$ or $k-1$ so they make sense). To recover the intuition from the previous discussion, the reader should picture that the random walk will visit the $i$ th vertex of the cycle at step $s$ whenever $X_{s}=(i, 1)$ or whenever $b>1$ and $X_{s}=(i+k+1-b, b)$. The second type of visits accounts for the described 'loops' which are necessary to maintain the homomorphism property.

For each $1 \leqslant j \leqslant k$, let $\mathcal{X}_{k, r t}^{(j)} \subseteq \mathcal{X}_{k, r t}$ consist of the tuples $(a, b)$ such that $a+b-1 \equiv j \bmod k$. The following lemma encapsulates the Markov-theoretic properties we need.

Lemma 10.4. Suppose $t, k$ are coprime. Then

(M1) $X$ is irreducible,

(M2) the (unique) stationary distribution $\pi$ of $X$ is given, for all a, by $\pi_{(a, 1)}=2 /(k r t)$, and $\pi_{(a, b)}=1 /(k r t)$ for $b \neq 1$,

(M3) if $r=1, X$ is aperiodic, and

(M4) if $r=k$, for any $1 \leqslant j \leqslant k$, the chain $X^{(j)}=\left\{X_{j+i k}\right\}_{i \geqslant 0}$ is supported on $\mathcal{X}_{k, r t}^{\left(j^{\prime}\right)}$ for some $1 \leqslant j^{\prime} \leqslant k$, is irreducible and aperiodic, and its stationary distribution $\pi^{(j)}$ is given by $\pi_{(a, 1)}^{(j)}=2 / k t$, and $\pi_{(a, b)}^{(j)}=1 / k$ for $b \neq 1$.

Proof. We begin by showing that $\mathcal{X}$ is irreducible. As discussed before, it suffices to show that the associated digraph $D=D\left(P, \mathcal{X}_{k, r t}\right)$ is strongly connected. Note that the directed cycle $C:=(1,1)(2,1) \cdots(r t, 1)$ covers all tuples of the form $(a, 1)$, and for any $a \in\{1, \ldots, r t\}$ we have $C_{a}:=(a, 1)(a, 2) \cdots(a, k-1)(a-1,1)$ is a directed cycle which covers all tuples of the type $(a, b)$ for fixed $a$. Then it is easy to construct, for any pair of given $(a, b),\left(a^{\prime}, b^{\prime}\right)$ a directed walk in $D$ from one to the other first by traversing $C_{a}$ to arrive at $(a-1,1)$, then follow $C$ to get to $\left(a^{\prime}, 1\right)$, and finally using $C_{a^{\prime}}$ to get to $\left(a^{\prime}, b^{\prime}\right)$.

The proof of item (M2) reduces to check the matrix equation $\pi=\pi P$ holds, which follows easily from a routine calculation.

To see that the chain is aperiodic when $r=1$, note that $C$ is a directed cycle of length $r t=t$ in $D$, and $C_{1}$ is a directed cycle of length $k$ in $D$. Since $k$ and $t$ are coprime, this readily implies the chain is aperiodic.

Finally, to see item (M4), note that when $r=k$ then the chain is periodic with period $k$. When $r=k$, the sets $\left\{\mathcal{X}_{k, k t}^{(j)}\right\}_{j=1}^{k}$ partition the state space, and it is easy to check that, for any $1 \leqslant j \leqslant k$, if $X_{i} \in \mathcal{X}_{k, k t}^{(j)}$, then $X_{i+k} \in \mathcal{X}_{k, k t}^{(j)}$. This shows $X^{(j)}$ is irreducible and aperiodic. Since the transition matrix of $X^{(j)}$ is given by (a restriction of) $P^{k}$, the stationary distribution is given by the restriction of $\pi$ to $\mathcal{X}_{k, k t}^{(j)}$ and normalising, which yields the result.

The algorithm will simulate a Markov chain $\left\{X_{i}\right\}_{i \geqslant 1}$ using the transition probabilities which were described before.

Algorithm 10.5. Let $p_{1}, \ldots, p_{q}$ be the ordered vertices of $P_{k, q}$. Let $c_{1}, \ldots, c_{r t}$ be the cyclically ordered vertices of $C_{k, r t}$. Initially, at step $s=1$ we will choose $X_{1} \in \mathcal{X}_{k, r t}$ according to the stationary distribution $\pi$ of the chain. If at step $s \geqslant 1$ we are given $X_{s}=(a, b)$ and $b=1$, then embed $p_{s} \mapsto c_{a}$, otherwise embed $p_{s} \mapsto c_{a-k+b-1}$ (indices understood modulo rt).

Lemma 10.6. Algorithm 10.5 yields a valid embedding of $P_{k, q}$ into $C_{k, r t}$.

Proof. For each $s \geqslant 1$, let $X_{s}=\left(a_{s}, b_{s}\right)$ be the states generated by the Markov chain used in Algorithm 10.5. Note that the only admissible transitions of the Markov chain force that once we have entered a state of type $(a, 2)$, we must use $k-1$ steps to arrive at $(a-1,1)$, after only which the option to use $(a-1,2)$ or $(a, 1)$ is possible. This observation entails that a sequence $\left(a_{s+1}, b_{s+1}\right) \cdots\left(a_{s+k}, b_{s+k}\right)$ of $k$ consecutive states of the Markov chain must necessarily have the form $X_{a, b} Y_{a-1, a^{\prime}} Z_{a^{\prime}, b^{\prime}}$ where $X_{a, b}$ is either empty or has the form 
$(a, b)(a, b+1) \cdots(a, k-1)$ for some $a$ and $2 \leqslant b \leqslant k-1, Y_{a-1, a^{\prime}}=(a-1,1)(a, 1)(a+$ $1,1) \cdots\left(a^{\prime}, 1\right)$, for some $a^{\prime}$ (possibly $a^{\prime}=a-1$ ), and $Z_{a^{\prime}, b^{\prime}}$ is either empty or has the form $\left(a^{\prime}, 2\right)\left(a^{\prime}, 3\right) \cdots\left(a^{\prime}, b^{\prime}\right)$ for some $2 \leqslant b \leqslant k-1$.

Thus, given $a, b, a^{\prime}, b^{\prime}$ which define $X_{a, b} Y_{a-1, a^{\prime}} Z_{a^{\prime}, b^{\prime}}$, Algorithm 10.5 will embed $k$ consecutive vertices of $P_{k, q}$ in the vertices

$$
c_{a-k+b-1} c_{a-k+b} \cdots c_{a-2} c_{a-1} c_{a} \cdots c_{a^{\prime}} c_{a^{\prime}-k+1} c_{a^{\prime}-k+2} \cdots c_{a^{\prime}-k-1+b^{\prime}}
$$

of $C_{k, t}$, with the indices always understood modulo $t$. Since there are $k$ steps, we must have $k=\left|X_{a, b}\right|+\left|Y_{a-1, a^{\prime}}\right|+\left|Z_{a^{\prime}, b^{\prime}}\right|=(k-b)+\left(a^{\prime}-a+2\right)+\left(b^{\prime}-1\right)=k-b+a^{\prime}-a+b^{\prime}+1$ and thus $a+b=a^{\prime}+b^{\prime}+1$. This implies that the vertices used in the embedding correspond to precisely $k$ consecutive vertices in $C_{k, r t}$, namely those from $c_{a^{\prime}-k+1}$ to $c_{a^{\prime}}$. Since this is true for any run of $k$ consecutive steps of the algorithm, this implies that the embedding is valid.

Now we are ready to prove Lemma 10.3.

Proof of Lemma 10.3. Let $p_{1}, \ldots, p_{q}$ be the ordered vertices of $P_{k, q}$, and let $\phi_{1}: V(H) \rightarrow$ $V\left(P_{k, q}\right)$ be a homomorphism as in the statement. In particular, for all $1 \leqslant i \leqslant r$, if we let $H_{i}=\phi_{1}^{-1}\left(\left\{p_{i}\right\}\right)$ then

$$
\left|H_{i}\right| \leqslant k\lceil\beta r n\rceil=: M .
$$

Now let $\phi_{2}: V\left(P_{k, q}\right) \rightarrow V\left(C_{k, r t}\right)$ be the random function given by Algorithm 10.5. By Lemma 10.6, $\phi_{2}$ is a graph homomorphism. Then the composition $\phi=\phi_{2} \circ \phi_{1}: V(H) \rightarrow$ $V\left(C_{k, r t}\right)$ defines a valid allocation from $H$ into $C_{k, r t}$. We now check that it satisfies the required properties with non-zero probability.

Recall that, for $X_{s}=(a, b)$, Algorithm 10.5 maps $p_{s} \mapsto c_{a}$ when $b=1$ and $p_{s} \mapsto c_{a-k+b-1}$ when $b>1$. So we have $\phi_{2}\left(p_{s}\right)=c_{i}$ for $1 \leqslant i \leqslant t$ precisely when $X_{s}=(i, 1)$ or $X_{s}=$ $(i+k-b+1, b)$ for $2 \leqslant b \leqslant k-1$. Motivated by this, we define

$$
S_{i}=\{(i, 1)\} \cup\{(i+k+1-b, b): 2 \leqslant b \leqslant k-1\} \subseteq \mathcal{X}_{k, t} .
$$

So $\phi_{2}\left(p_{s}\right)=c_{i}$ if and only if $X_{s} \in S_{i}$. Now define functions $f_{1}, \ldots, f_{r}: \mathcal{X}_{k, t} \rightarrow[0, M]$ such that, for all $1 \leqslant s \leqslant r$,

$$
f_{s}((a, b))= \begin{cases}\left|H_{s}\right| & \text { if }(a, b) \in S_{i}, \\ 0 & \text { otherwise. }\end{cases}
$$

Since $H_{s}$ will be embedded (via $\phi$ ) into $c_{i}$ if and only if $X_{s} \in S_{i}$, we have

$$
\left|\phi^{-1}\left(c_{i}\right)\right|=\sum_{s=1}^{r} f_{s}\left(X_{s}\right) .
$$

As the Markov chain $\left\{X_{i}\right\}_{i \geqslant 1}$ in Algorithm 10.5 was started from its stationary distribution $\pi$, it follows that $\operatorname{Pr}\left[X_{s}=(a, b)\right]=\pi_{(a, b)}$ for all $1 \leqslant s \leqslant r$ and $(a, b) \in \mathcal{X}_{k, t}$. Together with item (M2) we get $\operatorname{Pr}\left[X_{s} \in S_{i}\right]=1 / t$ for all $1 \leqslant s \leqslant r$. Therefore $\mathbf{E}_{\pi}\left[f_{s}\right]=\left|H_{s}\right| / t$ and thus

$$
\sum_{s=1}^{r} \mathbf{E}_{\pi}\left[f_{s}\right]=\sum_{s=1}^{r} \frac{\left|H_{s}\right|}{t}=\frac{n}{t} .
$$

We have $\sum_{s=1}^{r}\left|H_{s}\right|=n$ and $\left|H_{i}\right| \leqslant M=k\lceil\beta r n\rceil$. So by convexity $\sum_{s=1}^{r}\left|H_{s}\right|^{2}$ is maximised when as many $\left|H_{i}\right|$ 's as possible take the value $M$. In consequence,

$$
\sum_{s=1}^{r}\left|H_{s}\right|^{2} \leqslant 2 k \beta r^{2} n^{2} .
$$

Now, suppose we are in the case $r=1$. Then the chain $\left\{X_{i}\right\}_{i \geqslant 1}$ is irreducible and aperiodic by item (M1) and item (M3). Applying Theorem 10.2 gives $C=C(k, t)$ depending on $k, t$ 
only. We apply inequality (10.1) with $\xi n / t,\left|H_{s}\right|$ playing the roles of $\varepsilon, b_{i}-a_{i}$ respectively, to get

$$
\begin{aligned}
\operatorname{Pr}\left[|| \phi^{-1}\left(c_{i}\right)\left|-\frac{n}{t}\right|>\frac{\xi n}{t}\right] & \stackrel{(10.2),(10.3)}{=} \operatorname{Pr}\left[\left|\sum_{s=1}^{r} f_{s}\left(X_{s}\right)-\sum_{s=1}^{r} \mathbf{E}_{\pi}\left[f_{s}\right]\right|>\frac{\xi n}{t}\right] \\
& \stackrel{(10.1)}{\leqslant} 2 \exp \left(-\frac{C \xi^{2} n^{2}}{2 t^{2} \sum_{s=1}^{r}\left|H_{s}\right|^{2} / 4}\right) \\
& \stackrel{(10.4)}{ } \leqslant 2 \exp \left(-\frac{C \xi^{2}}{t^{2} k \beta}\right)<\frac{1}{t}
\end{aligned}
$$

where we used $\beta \ll \xi, 1 / t, 1 / k$ in the last step. Using a union bound over all the $t$ possible choices of $c_{i}$, we deduce that with positive probability $\left|\phi^{-1}\left(c_{i}\right)\right|=(1 \pm \xi) n / t$ for all $c_{i} \in$ $V\left(C_{k, t}\right)$, as desired.

Now suppose $r=k$. Fix $j \in\{1, \ldots, k\}$. By assumption, we have that $\left|\phi^{-1}\left(P_{j}\right)\right|=n$. The main point here is that (without loss of generality, after relabelling vertices of the cycle if necessary) the vertices in $P_{j}$ must always be mapped to vertices of the cycle of type $X_{j+k i}$. Since the chain $\left\{X_{j+i k}\right\}_{i \geqslant 0}$ is irreducible and aperiodic by item (M4), we can apply (10.1) using this new chain and conclude in similar way as the $r=1$ case.

10.5. A cycle to correct imbalances. Here we find an allocation of a tight cycle into a $k$-graph $\mathcal{J}$ such that $(R, \mathcal{J})$ forms a robust Hamilton framework, with the additional restriction that the number of vertices allocated to each cluster is proportionally very close to the sizes prescribed by a given $(1+\varepsilon)$-balanced partition. Its proof will be an application of Lemma 8.3 (Lemma for $C$ ).

Lemma 10.7 (Imbalance-correcting Lemma for $C$ ). Let $1 / n \ll \xi \ll \pi \ll 1 / t, \varepsilon \ll \alpha \ll$ $\mu, 1 / k$ and let $r \in\{1, k\}$. Let $\mathcal{U}$ be an $(n, r)$-sized partition, and let $G$ be a U-partite graph. Let $\mathcal{V}=\left\{V_{i}\right\}_{i=1}^{t}$ be a U-refining $(1+\varepsilon)$-balanced partition of $V(G)$, with $t$ clusters inside each cluster of $\mathcal{U}$, such that $G$ is $\mathcal{V}$-partite. Let $\mathcal{W}$ be the $(t, r)$-sized partition of $R$ induced by $(\mathcal{V}, \mathcal{U})$. Suppose $(R, \mathcal{J})$ is a $(\mu / 2)$-robust $\mathcal{W}$-partite Hamilton framework and $(\mu / 2,2 / n, \mathcal{W})$ cluster-matchable. If $r=1$, suppose in addition that $(R, \mathcal{J})$ is $(\mu / 2)$-robust aperiodic. Let $V(R)=\{1, \ldots, t\}$ and suppose $\mathcal{J}^{\prime}=(1,2, \ldots, t)$ is a tight Hamilton cycle in $\mathcal{J}$.

Then there is $\ell \leqslant 2 r t^{2} \xi^{-1}$ coprime to $k$ and a homomorphism $\phi: V\left(C_{k, r \ell}\right) \rightarrow V(R)$ such that

(J1) for every $i \in V(R)$,

$$
\frac{\left|\phi^{-1}(i)\right|}{\ell}=\frac{\left|V_{i}\right|}{n} \pm \xi
$$

(J2) for every $i \in V(R)$ there exists $\tilde{X}_{i} \subseteq \phi^{-1}(i) \subseteq V\left(C_{k, r \ell}\right)$ such that $\left|\tilde{X}_{i}\right| \geqslant \alpha\left|\phi^{-1}(i)\right|$ and for each $x \in \tilde{X}_{i}$ and $x y, y z \in E\left(C_{k, \ell}\right)$, with $\phi(y)=j$ and $\phi(z)=l$, we have $i l, j l \in E\left(R^{\prime}\right)$ where $R^{\prime}=\partial_{2} \mathcal{J}^{\prime}$, and

(J3) for every $i \in V(R)$ there exists $Z_{i} \subseteq \phi^{-1}(i) \subseteq V\left(C_{k, \ell}\right) \backslash \tilde{X}_{i}$ such that $\left|Z_{i}\right| \geqslant \pi\left|\phi^{-1}(i)\right|$ and for each $x \in Z_{i}, y \in N_{C_{k, \ell}}(x)$, we have $\phi(y) \in\{i-k+1, i-k+2, \ldots, i-1\}$.

Proof. Choose the largest $\ell$ such that $\ell \leqslant 2 r t^{2} \xi^{-1}$ and $\ell$ is coprime with $k$. By choice, we can assume $1 / \xi \ll 1 / \ell \ll 1 / t, \varepsilon, \alpha, \mu, 1 / k$. In particular, $\ell \geqslant r t^{2} \xi^{-1}$. Next, we choose $\ell_{1}, \ldots, \ell_{r t} \geqslant 0$ such that

(i) If $r=1$, then $\sum_{i=1}^{t} \ell_{i}=\ell$,

(ii) if $r=k$, then for each $1 \leqslant j \leqslant k, \sum_{i=0}^{t-1} \ell_{j+i k}=\ell$, and

(iii) the quantity $\sum_{i=1}^{r t}\left|\frac{\ell_{i}}{\ell}-\frac{\left|V_{i}\right|}{n}\right|$ is minimised among all the possible choices.

Note that we cannot have $\frac{\ell_{i}}{\ell}-\frac{\left|V_{i}\right|}{n}>t / \ell$ for some $i$, as otherwise there exists some $j$ such that $j \equiv i \bmod r$ and $\frac{\ell_{j}}{\ell}-\frac{\left|V_{j}\right|}{n} \leqslant-1 / \ell$. Adding one to $\ell_{j}$ and substracting one from $\ell_{i}$ yields 
a new valid sequence where the objective sum is strictly minimised, a contradiction. An analogous argument shows that $\frac{\ell_{i}}{\ell}-\frac{\left|V_{i}\right|}{n} \geqslant-t / \ell$ for all $i$ as well, thus we have

$$
\sum_{i=1}^{r t}\left|\frac{\ell_{i}}{\ell}-\frac{\left|V_{i}\right|}{n}\right| \leqslant \frac{r t^{2}}{\ell} \leqslant \xi .
$$

Let $G^{\prime}$ be the graph obtained from $R$ by blowing-up each $i \in V(R)$ by $\ell_{i}$ vertices, let $\mathcal{V}^{\prime}$ be the natural associated partition of $V\left(G^{\prime}\right)$ where $\left|V_{i}^{\prime}\right|=\ell_{i}$ for all $i$; and let $\mathcal{U}^{\prime}$ be the $(\ell, r)$-partition of $V\left(G^{\prime}\right)$ where each cluster $U^{\prime} \in \mathcal{U}^{\prime}$ corresponds of the blown-up vertices of $R$ lying in the same cluster of the partition $\mathcal{W}$ of $V(R)$.

We check the hypothesis of Lemma 8.3 hold with $G^{\prime}, \mathcal{V}^{\prime}, \mathfrak{U}^{\prime}, \ell, 2 \varepsilon, \mu / 2$ playing the roles of $G, \mathcal{V}, \mathcal{U}, n, \varepsilon, \mu$, respectively. By construction, $\mathcal{V}^{\prime}$ is an $\mathcal{U}^{\prime}$-refining partition. Since $\mathcal{V}=$ $\left\{V_{1}, \ldots, V_{r t}\right\}$ is a $(1+\varepsilon)$-balanced partition, together with eq. (10.5) we deduce that $\mathcal{V}^{\prime}$ is a $(1+2 \varepsilon)$-balanced partition. By choice, $\left(G^{\prime}, \mathcal{V}^{\prime}\right)$ is an $R$-partition. If $\mathcal{W}^{\prime}$ is the $(t, r)$ sized partition of $R$ induced by $\left(\mathcal{V}^{\prime}, \mathcal{U}^{\prime}\right)$, note that $\mathcal{W}^{\prime}=\mathcal{W}$. By assumption, $(R, \mathcal{J})$ is a $(\mu / 2)$-robust $\mathcal{W}$-partite Hamilton framework on $t$ framework which is $(\mu / 2,2 / n, \mathcal{W})$-clustermatchable; and if $r=1$ we also assume $(R, \mathcal{J})$ is $(\mu / 2)$-robust aperiodic. In particular, since $\ell \leqslant n,(R, \mathcal{J})$ is $(\mu / 2,2 / \ell, \mathcal{W})$-cluster-matchable. By assumption, $\mathcal{J}^{\prime} \subseteq \mathcal{J}$ is a tight Hamilton cycle, which satisfies $\Delta\left(\mathcal{J}^{\prime}\right) \leqslant k \leqslant k^{2}+1$.

The application of Lemma 8.3 yields a size-compatible (with $\mathcal{V}^{\prime}$ ) vertex partition $X=$ $\left\{X_{i}\right\}_{1 \leqslant i \leqslant t}$ of the vertex set of the $(k-1)$ th power of a Hamilton cycle $C=C_{k, r \ell}$ such that $(C, X)$ is an $R$-partition. We also obtain a family of subsets $\tilde{X}$ such that $\tilde{X}$ is an $\left(2 \alpha, R^{\prime}\right)$ buffer for $((C, X)$. Note that the partition $\mathcal{X}$ defines a natural homomorphism $\phi: V(\mathcal{C}) \rightarrow$ $V(R)$ where $\phi^{-1}(i)=X_{i}$. Now item (J1) follows from eq. (10.5), and item (J2) follows from the existence of $\tilde{X}$. Finally, to obtain item (J3) consider an $i \in V(R)$ and note that $K=\{i-k+1, i-k+2, \ldots, i-1\}$ is an edge in $\mathcal{J}^{\prime} \subseteq \mathcal{J}$. Hence by item (C3), there is a $(k-1)$ th power of a path $P_{K} \subseteq C\left[\bigcup_{i \in K} X_{i}\right]$ of length $\pi n \geqslant \pi \frac{t n}{2} \frac{\left|V_{i}\right|}{n} \geqslant 2 \pi\left|\phi^{-1}(i)\right|$. We therefore may choose a $Z_{i} \subseteq \phi^{-1}(i) \subseteq V\left(C_{k, r \ell}\right)$ to contain the pre-image of the $\pi\left|\phi^{-1}(i)\right|$ middle vertices of $P_{K}$. Note that we can assume that $Z_{i} \cap \tilde{X}_{i}=\emptyset$ by replacing $\tilde{X}_{i}$ with $\tilde{X}_{i} \backslash Z_{i}$, turning the $\left(2 \alpha, R^{\prime}\right)$-buffer $\tilde{X}$ into an $\left(\alpha, R^{\prime}\right)$-buffer.

10.6. Proof of the Lemma for $H$. Finally, we show the main result of Section 10.

Proof of Lemma 9.2. We carry out the proofs for the cases (i) to (iii) simultaneously. Initially, assume $H$ admits an ordering with bandwidth at most $\beta n$ and a $(z, \beta)$-zero-free $(k+1)$ colouring, and that $(R, \mathcal{J})$ is only a Hamilton framework (not zero-free). If $r=k$, we furthermore assume that $H$ admits an equitable $k$-colouring. The proof has four steps. At the end of Step 3 we will be done with the embedding if $H$ has no zero-coloured vertices, which covers cases (i) and (iii). In Step 4, we will introduce the extra hypothesis of zero-freeness to $(R, \mathcal{J})$ to continue, and this will finish the proof in all cases.

Step 1: Finding an imbalance-correcting cycle in $R$. Recall that $\mathcal{J}^{\prime} \subseteq \mathcal{J}$ is a tight Hamilton cycle whose cyclic order we assume to be $1,2, \ldots, r t$. Let $R^{\prime}=\partial_{2} \mathcal{J}^{\prime}$.

First, apply Lemma 10.7 with $\xi^{2}, 3 \pi$ and $3 \alpha$ in place of $\xi, \pi$ and $\alpha$, respectively, to obtain $\ell \leqslant 2 t^{2} \xi^{-2}$ coprime to $k$ and a homomorphism $\phi_{3}: V\left(C_{k, \ell}\right) \rightarrow V(R)$ satisfying

(J1) for every $i \in V(R)$,

$$
\frac{\left|\phi_{3}^{-1}(i)\right|}{\ell}=\frac{\left|V_{i}\right|}{n} \pm \xi^{2}
$$

(J2) for every $i \in V(R)$ there exists $\tilde{Y}_{i} \subseteq \phi_{3}^{-1}(i) \subseteq V\left(C_{k, \ell}\right)$ such that $\left|\tilde{Y}_{i}\right| \geqslant 3 \alpha\left|\phi_{3}^{-1}(i)\right|$ and for each $x \in \tilde{Y}_{i}$ and $x y, y z \in E\left(C_{k, \ell}\right)$, with $\phi(y)=j$ and $\phi(z)=l$, we have $i j, j l \in E\left(R^{\prime}\right)$

(J3) for every $i \in V(R)$, there exists $\tilde{Z}_{i} \subseteq \phi_{3}^{-1}(i) \subseteq V\left(C_{k, \ell}\right) \backslash \tilde{Y}_{i}$ such that $\left|\tilde{Z}_{i}\right| \geqslant 3 \pi\left|\phi_{3}^{-1}(i)\right|$ and for each $z \in \tilde{Z}_{i}, y \in N_{C_{k, \ell}}(z)$ we have $\phi_{3}(y) \in\{i-k+1, i-k+2, \ldots, i-1\}$. 
Step 2: Preparing $H$. Let $H_{0} \subseteq H$ be the set of 0-coloured vertices of $H$ and $H_{1}=V(H) \backslash H_{0}$. Note that $H_{0}$ could be empty. Let $q=\left\lceil\beta^{-1}\right\rceil+k-1$. Recall that $P_{k, q}$ is the $(k-1)$ th power of a path with vertices $p_{1}, \ldots, p_{q}$. By Lemma 10.1, there exists a homomorphism $\phi_{1}: V(H) \rightarrow V\left(P_{k, r}^{+}\right)$such that

(P1) $\phi_{1}^{-1}(\{*\})=H_{0}$,

(P2) $\phi_{1}$ restricted to $V(H) \backslash H_{0}$ is an at most $(k\lceil\beta n\rceil)$-to-one homomorphism to $P_{k, q}$, and

(P3) there exist $s \geqslant 0$ and indices $1 \leqslant i_{1} \leqslant i_{2} \leqslant \cdots \leqslant i_{s} \leqslant q$ such that

(i) $i_{j+1}-i_{j} \geqslant z$ for all $1 \leqslant j<s$,

(ii) for every $v \in V(H)$ adjacent to a vertex in $H_{0}$, there exists $1 \leqslant j<s$ such that $\phi_{1}(v) \in\left\{p_{i_{j}-1}, \ldots, p_{i_{j}+k}\right\}$.

(P4) Moreover, if $r=k$, then $\phi_{1}$ maps $n$ vertices to each $P_{j}:=\left\{p_{i}: i \in j+k \mathbb{N}\right\}$.

We remark that the final part follows implicitly, as (by assumption) $H$ admits an equitable $k$-colouring in this case. Also note that item (P3)(i) implies in particular that $s \leqslant q z^{-1}+1$. From the definition of $(z, \beta)$-zero-free $(k+1)$-colourings we can deduce that

(P5) $\left|H_{0}\right| \leqslant s\lceil\beta n\rceil \leqslant\left(q z^{-1}+1\right)\lceil\beta n\rceil \leqslant 2 z^{-1} n \leqslant \xi^{2} n$,

where we used $1 / z \ll \xi$ in the last inequality.

Now we partition $H$ in vertex-disjoint subgraphs which we will allocate separately. To ease notation, for $i<1$ or $i>q$ define $p_{i}=H_{i}=\emptyset$. For each $a<b \in \mathbb{Z}$, let $H_{[a, b]}=\phi_{1}^{-1}\left(\left\{p_{i}: a \leqslant\right.\right.$ $i \leqslant b\}) \subseteq H_{1}$, in other words $H_{[a, b]}$ consists of the preimages of $b-a+1$ consecutive vertices of $P_{k, q}$, from $p_{a}$ to $p_{b}$. Let $\omega=k t^{k}$. For each $1 \leqslant j \leqslant s$ we define $a_{j}, b_{j}, c_{j}, d_{j} \in \mathbb{Z}$ as

$$
\begin{aligned}
a_{j} & =i_{j}-2-\omega+k, & b_{j} & =i_{j}+k-3, \\
c_{j} & =i_{j}+2, & d_{j} & =i_{j}+1+\omega,
\end{aligned}
$$

These numbers and tuples were selected so that the intervals $\left[a_{j}, b_{j}\right]$ and $\left[c_{j}, d_{j}\right]$ have $\omega$ elements each; and the last $k-1$ elements of $\left[a_{j}, b_{j}\right]$ (resp. the first $k-1$ elements of $\left[c_{j}, d_{j}\right]$ ) correspond to the first (resp. last) $k-1$ elements of $\left\{i_{j}-1, \ldots, i_{j}+k\right\}$. Since $1 / z \ll 1 / t, k$, we have $z>2 \omega-k+4$. Together with item (P3)(i), we deduce that for each $1 \leqslant j \leqslant s$, the intervals $\left[a_{j}, d_{j}\right]$ are pairwise disjoint. For each $1 \leqslant j \leqslant s$, let

$$
F_{j}=H_{\left[a_{j}+k, d_{j}-k\right]} \text { and } H^{\prime}=H_{1} \backslash \bigcup_{1 \leqslant j \leqslant s} F_{j} .
$$

Note that $H^{\prime}, F_{1}, \ldots, F_{s}$ and $H_{0}$ partition the vertex set of $H$, and $H_{0}$ contains all zerocoloured vertices of $H$.

Step 3: Embedding the non-zero coloured vertices. We will embed $H^{\prime}$ first using Lemma 10.3 (Randomised allocation for $H$ ), we proceed to check the necessary hypothesis. Let $n^{\prime}$ be the number of vertices in $H^{\prime}$. First, note that by item (P2), for all $1 \leqslant j \leqslant s$ we have $\left|F_{j}\right| \leqslant(2 \omega+2 k) k\lceil\beta n\rceil$. Thus, together with item (P5), we deduce

$$
n^{\prime}=\left|H^{\prime}\right|=n-\left|H_{0}\right|-\sum_{j=1}^{s}\left|F_{j}\right| \geqslant\left(1-\xi^{2}\right) n-s(2 \omega+2 k) k\lceil\beta n\rceil \geqslant\left(1-2 \xi^{2}\right) n,
$$

where we used $s \leqslant 2 \beta^{-1}, \omega=k t^{k}$, and $1 / z, \beta \ll \xi$ in the last step. Since $\ell \leqslant 2 t^{2} \xi^{-2}$, $q=\left\lceil\beta^{-1}\right\rceil+k-1$ and $\beta \ll \xi, 1 / t$, we can assume the hierarchy $1 / n^{\prime} \ll \beta, 1 / q \ll \xi, 1 / \ell, 1 / k$ is satisfied. Also, $\ell$ and $k$ are coprime. Note that the restriction of $\phi_{1}$ to $H^{\prime}$ is a homomorphism $\phi_{1}^{\prime}$ from $H^{\prime}$ to $P_{k, q}$ which is at most $(k\lceil\beta n\rceil)$-to-one,. Hence $\phi_{1}^{\prime}$ is at most $\left(k\left\lceil 2 \beta n^{\prime}\right\rceil\right)$-toone. Finally, by item (P4), it follows that if $r=k$, then $\left|\phi_{1}^{-1}\left(P_{j}\right)\right|=n$. Thus we can apply Lemma 10.3 with $n^{\prime}, 2 \beta, \xi^{2}, H^{\prime}, \phi_{1}^{\prime}$ in place of $n, \beta, \xi, H, \phi_{1}$ respectively, to obtain a homomorphism $\phi_{2}: V\left(H^{\prime}\right) \rightarrow V\left(C_{k, \ell}\right)$ which is $\left(\left(1 \pm \xi^{2}\right) \frac{n^{\prime}}{\ell}\right)$-to-one. Since $n^{\prime}=\left(1 \pm 2 \xi^{2}\right) n$, it follows that $\phi_{2}$ is $\left(\left(1 \pm 3 \xi^{2}\right) \frac{n}{\ell}\right)$-to-one.

Then $\Phi=\phi_{3} \circ \phi_{2}$ is a homomorphism from $H^{\prime}$ to $R$. This naturally defines a partition $X^{\prime}$ of $V\left(H^{\prime}\right)$ by $X_{i}^{\prime}=\Phi^{-1}(i)$, and so $\left(H^{\prime}, X^{\prime}\right)$ is an $R$-partition. For all $1 \leqslant i \leqslant t$, let $\tilde{X}_{i}^{\prime}=\phi_{2}^{-1}\left(\tilde{Y}_{i}\right)$ with $\tilde{X}^{\prime}=\left\{\tilde{X}_{i}^{\prime}\right\}_{i=1}^{t r}$, and let $Z_{i}=\phi_{2}^{-1}\left(\tilde{Z}_{i}\right)$ with $z=\left\{\tilde{Z}_{i}\right\}_{i=1}^{t r}$ where the sets $\tilde{Y}_{i}, \tilde{Z}_{i}$ are from 
items (J2) and (J3). From items (J1) to (J3), the fact that $\phi_{2}$ is $\left(\left(1 \pm 3 \xi^{2}\right) \frac{n}{\ell}\right)$-to-one and $\xi \ll \pi, \alpha$, we deduce that

(H1) $\tilde{X}^{\prime}$ is a $\left(2 \alpha, R^{\prime}\right)$-buffer for $\left(H^{\prime}, X^{\prime}\right)$,

(H2) for each $1 \leqslant i \leqslant t, Z_{i} \subseteq X_{i}$ such that $\left|Z_{i}\right| \geqslant 2 \pi\left|X_{i}^{\prime}\right|$, and for each $z \in Z_{i}, N_{H}(z) \subseteq$ $X_{i-1} \cup \cdots \cup X_{i-k+1}$, and

(H3) for each $i \in V(R),\left|X_{i}^{\prime}\right|=\left|V_{i}\right| \pm 4 \xi^{2}$.

At this point of the proof we stop to conclude if the input graph $H$ was $k$-colourable, which covers cases (i) and (iii) of Lemma 9.2. Indeed, if $H$ is $k$-colourable then $H_{0}=\emptyset$ and $s=0$. Therefore, $H^{\prime}=H$. Hence, $\Phi$ is a homomorphism from $H$ to $R$ and $X^{\prime}$ is a partition of $V(H)$ which satisfies the required properties.

Step 4: Embedding the zero-coloured vertices. From now on, we can assume $H^{\prime} \neq H$ and that $(R, \mathcal{J})$ is actually a $(\mu / 2)$-robust zero-free Hamilton framework. In particular, we assume $r=1$. This implies that there exists a copy of a $(k+1)$-clique $K$ in $\mathcal{J}$, let $c_{1}, \ldots, c_{k+1}$ be its vertices. Recall that we need to extend the homomorphism $\Phi$ to allocate the remaining vertices, which are in $H_{0}$ and $F_{1}, \ldots, F_{s}$.

Fix $1 \leqslant j \in s$. The homomorphism $\Phi$ has already allocated $H^{\prime}$, including the vertices in $H_{\left[a_{j}, a_{j}+k-1\right]}$ and $H_{\left[d_{j}-k+1, d_{j}\right]}$ in $R$, whose images correspond to tuples denoted by $e_{1}, e_{4} \in$ $V(R)^{k-1}$. Let $e_{2}=\left(c_{1}, \ldots, c_{k-1}\right)$ and $e_{3}=\left(c_{4}, \ldots, c_{k}, c_{1}, c_{2}\right)$. By Proposition 7.4, $R$ contains a walk $W_{1}=\left(w_{1}, \ldots, w_{\omega}\right)$ from $e_{1}$ to $e_{2}$, and a walk $W_{2}=\left(w_{1}^{\prime}, \ldots, w_{\omega}^{\prime}\right)$ from $e_{3}$ to $e_{4}$ where $\omega=k t^{k}$. We allocate $H_{\left[a_{j}, b_{j}\right]}$ in $R$ by assigning $\phi_{1}^{-1}\left(p_{a_{j}+i-1}\right)$ to $w_{i} \in V(R)$, for all $1 \leqslant i \leqslant \omega$, and we allocate $H_{\left[c_{j}, d_{j}\right]}$ in $R$ by assigning $\phi_{1}^{-1}\left(p_{c_{j}+i-1}\right)$ to $w_{i}^{\prime} \in V(R)$, for all $1 \leqslant i \leqslant \omega$.

Our choice of $a_{j}, c_{j}, e_{1}, e_{2}, e_{3}, e_{4}$ and $W_{1}, W_{2}$ ensures that this allocation is well-defined. Doing this with all $F_{j}$, this extends the embedding of $H^{\prime}$ to an embedding of $H_{[1, q]}=H-H_{0}$. It only remains to allocate the vertices in $H_{0}$, we do that as follows.

By item (ii), the vertices in $H_{0}$ are joined, in $H$, only to vertices in $\phi_{1}^{-1}\left(\left\{p_{i_{j}-1}, \ldots, p_{i_{j}+1}\right\}\right)$ for $1 \leqslant j \leqslant s$. By the allocation of $F_{j}$, these vertices are allocated to vertices which are all in $\left\{c_{1}, \ldots, c_{k}\right\}$. Thus we get a valid allocation of $H$ to $R$ by setting $\Phi\left(H_{0}\right)=c_{k+1}$. Finally, by inequality (10.7) this (and the above steps) changes the allocation in any given cluster by at most $2 \xi^{2} n$. In combination with items (H1) to (H3), this final allocation satisfies all the required properties of Lemma 9.2.

\section{Conclusion}

In this paper, we investigated a set of general properties that guarantee the existence of (powers of) Hamilton cycles as well as containment of large graphs of sublinear bandwidth. As an application we recovered several classic results and also proved new results. Besides getting a clearer picture of what Hamiltonicity in dense graphs is about, we hope that our work will pave for future results in this line of research. We finish with some reflections and present a few problems that appear to be within reach.

11.1. Chvátal-type degree conditions. It would be very interesting to characterise the degree sequences that witness the existence of the $(k-1)$ th power of a Hamilton cycle. For $k=2$, this was done by Chvátal [16], who showed that a degree sequence $d_{1} \leqslant \ldots \leqslant d_{n}$ that satisfies $d_{i} \geqslant i+1$ or $d_{n-i} \geqslant n-i$ for every $i<n / 2$ guarantees a Hamilton cycle. Moreover, for every sequence $a_{1} \leqslant \cdots \leqslant a_{n}$ such that $a_{h} \leqslant i$ and $a_{n-h} \leqslant n-i-1$ for some $h<n / 2$ there exists a non-Hamiltonian graph whose degree sequence $d_{1} \leqslant \cdots \leqslant d_{n}$ satisfies $d_{i} \geqslant a_{i}$ for all $1 \leqslant i \leqslant n$. Up to an error term, these conditions were extended to ensure the existence of bipartite spanning graphs of sublinear bandwidth by Knox and Treglown [41] (as a corollary of Theorem 2.12).

For $k \geqslant 3$ much less is known. Balogh, Kostochka and Treglown [3, Proposition 17] asked whether a degree sequence, satisfying $d_{i} \geqslant \frac{k-2}{k} n+i$ or $d_{n-i(k-1)} \geqslant n-i$ for all $i \leqslant n / k$, would guarantee the existence of a $k$-clique factor. They also provided constructions showing that the value of the second part of the condition cannot be lowered. It is conceivable that graphs whose degrees lie well-above this sequence allow for a Bandwidth Theorem. 
Conjecture 11.1. For $\mu>0$ and $n$ large enough, let $G$ be a graph with degree sequence $d_{1} \leqslant \ldots \leqslant d_{n}$. Suppose that $d_{i} \leqslant \frac{k-2}{k} n+i+\mu n$ or $d_{n-i(k-1)} \leqslant n-i+\mu n$ for every $i \leqslant n / k$. Then $(G, H)$ is a zero-free Hamilton framework where $H=K_{k}(G)$.

11.2. Multipartite graphs. Theorem 2.10 provides the (approximate) minimum degree conditions for tight Hamilton cycles in multipartite graphs, where the minimum degree is defined relative to each of the clusters. Do other theorems have partite versions? There are Pósa-type [61] and Chvátal-type [54, Corollary 20] degree conditions which ensure Hamilton cycles in balanced bipartite graphs. If the degree conditions on those theorems is augmented by an $o(n)$ term and the host graphs taken to be large enough, then our results on frameworks apply, and the output is strengthened from Hamiltonicity to the containment of bipartite graphs of sublinear bandwidth. It is certainly conceivable that these results can be extended to powers of cycles in multipartite graphs. More generally, it would be interesting to determine under which general conditions the multi-partite and ordinary form of such problems are equivalent.

In a different direction than Theorem 2.10, Lo and the second author [56, Corollary 1.5] showed a result for $k$-clique factors in balanced $k$-partite graphs where the overall minimum degree is controlled (instead of the minimum degree of every vertex to every other cluster).

Theorem 11.2. Let $1 / n \ll 1 / k, \mu$ with $k \geqslant 2$. Let $G$ be a balanced $k$-partite graph on $k n$ vertices with $\delta(G) \geqslant(k-3 / 2+\mu) n$. Then $G$ has a $k$-clique factor.

The result is best possible apart from the $\mu n$ term. It seems sensible to expect this result could be combined with Theorem 3.5 to prove such graphs also contain powers of Hamilton cycles, or more generally, are partite $(\beta, \Delta, k)$-Hamiltonian. However, we were unable to decide if $K_{k}(G)$ is tightly connected for such graphs.

11.3. Robust expansion. Robust Hamilton frameworks provide a generalisation of robust expansion, in the sense that they present sufficient conditions for the existence of powers of cycles and sublinear bandwidth graphs of chromatic number larger than 2. The concept of robust expanders has proven to be useful to tackle problems about Hamilton cycles that go beyond their simple existence, we survey a few of them next.

Firstly, robust expanders admit a natural extension to digraphs [52] and have been used to find directed Hamilton cycles. Secondly, robust expanders have been applied to problems of algorithmic nature, such as designing efficient parallel algorithms to find Hamilton cycles in dense graphs [15], or problems concerning the domination ratio of the Asymmetric TSP problem [48, Corollary 1.5]. Thirdly, in some situations dense graphs that are not robust expanders can be vertex-partitioned into a bounded number of parts, each of which is robust. This can be applied to find Hamilton cycles under additional assumptions as high vertex-connectivity [46] or vertex-transitivity [14]. Finally, robust expanders have led to the resolution of long-standing problems about decompositions of graphs and digraphs into Hamilton cycles (see [49] for a comprehensive survey).

It would be interesting to understand whether Hamilton frameworks can be adapted and used to generalise these results. In the setting of minimum degree conditions, a step in this direction has been done by Condon, Kim, Kühn and Osthus [17] to find approximate decompositions into sublinear bandwidth graphs. Finally, another interesting problem is whether characterisations such as in Theorem 5.29 (Tutte's theorem) are also possible for more complicated structures such as clique factors.

11.4. Mitigating conflicts. In many applications, the given host graph contains conflicting edges, and the guest graph is not allowed to include any two conflicting edges. An interesting special case of this problems arises when the conflicts between edges form an equivalence relation. So every edge belongs to an equivalence class, which we refer to as its colour. Put this way, the host graph is edge-coloured and we have to find a rainbow copy of the guest graph, meaning that all its edges have distinct colours. Apart from being a natural 
question on its own, this encodes many combinatorial problems such as the Ryser-BrualdiStein Conjecture [11] on transversals in latin squares and Ringel's conjecture [60] about partitioning a graph into edge-disjoint copies of a tree.

Coulson and Perarnau [18] proved that any locally bounded edge-colouring of a subgraph $G \subseteq K_{n, n}$ with $\delta(G) \geqslant n / 2$ has a rainbow perfect matching. One can therefore wonder, whether this is an instance of a more general phenomenon, namely that any Dirac-type problem asymptotically allows for a rainbow version? For minimum degree conditions, this was confirmed by Glock and Joos [30] who proved a Bandwidth Theorem in the rainbow setting. We believe that our results can be generalised in a similar way.

Conjecture 11.3. For $k, \Delta \in \mathbb{N}, \mu>0$, and $r=1$ or $r=k$ there are $\nu, \beta, z$ and $n_{0} \in \mathbb{N}$ with the following property. Let $G$ be as in the assumptions of Theorem 3.5. Suppose that $G$ has in addition an edge-colouring such that every colour appears on at most $\nu$ n edges. Then $G$ contains rainbow copies of the graphs detailed in Theorem 3.5 (i)-(iii).

11.5. Algorithmic aspects. For fixed $k$, one can search for a $k$-uniform Hamilton framework in polynomial time (see Appendix B.1). On the other hand, searching for a Hamilton cycle or any of its powers is NP-complete in general. It is not clear whether one can efficiently search for (or even decide about the existence of) a $\mu$-robust $k$-uniform Hamilton framework. However, it seems reasonable that our proof method could yield an efficient algorithm which finds a $(k-1)$ th power of a cycle or a given $k$-colourable sublinear-bandwidth spanning graph, or otherwise returns that the input graph is not a $\mu$-robust $k$-uniform Hamilton framework. This could be done using the algorithmic versions of the Regularity Lemma and the Blow-Up Lemma (see [43]), subject to the derandomisation of the parts where we rely on probabilistic arguments.

11.6. Hypergraphs. Finally, it is natural to ask whether these type of results generalise to hypergraphs. On the practical side, first steps in this direction were undertaken by Schülke [67] for tight cycles under codegree sequence conditions in 3-uniform hypergraphs and by and Bowtell and Hyde [9] for perfect matchings under minimum vertex degree conditions in 3 -uniform graphs. On the theoretical side, Hamilton frameworks minimum degree conditions for tight Hamilton cycles have been proposed in our earlier work [53]. We plan to revisit this line of research in the near future.

Acknowledgments. We thank Andrew Treglown, Katherine Staden and Louis DeBiasio for helpful discussions and feedback.

\section{REFERENCES}

1. P. Allen, J. Böttcher, J.Julia Ehrenmüller, and A. Taraz, The bandwidth theorem in sparse graphs, Adv. Comb. (2020), Paper No. 6, 60.

2. P. Allen, J. Böttcher, H. Hàn, Y. Kohayakawa, and Y. Person, Blow-up lemmas for sparse graphs, arXiv:1612.00622 (2016).

3. J. Balogh, A. V. Kostochka, and A. Treglown, On perfect packings in dense graphs, arXiv:1110.3490 (2011).

4. _ On perfect packings in dense graphs, Electron. J. Combin. 20 (2013), no. 1, Paper 57, 17.

5. D. P. Bertsekas, Convex optimization theory, Athena Scientific, Nashua, NH, 2009.

6. J. Böttcher and S. Müller, Forcing spanning subgraphs via Ore type conditions, European Conference on Combinatorics, Graph Theory and Applications (EuroComb 2009), Electron. Notes Discrete Math., vol. 34, Elsevier Sci. B. V., Amsterdam, 2009, pp. 255-259.

7. J. Böttcher, M. Schacht, and A. Taraz, Spanning 3-colourable subgraphs of small bandwidth in dense graphs, J. Combin. Theory Ser. B 98 (2008), no. 4, 752-777.

8. __ Proof of the bandwidth conjecture of Bollobás and Komlós, Math. Ann. 343 (2009), no. 1, 175205.

9. C. Bowtell and J. Hyde, A degree sequence strengthening of the vertex degree threshold for a perfect matching in 3-uniform hypergraphs, arXiv:2008.12222 (2020).

10. S. Brandt, H. Broersma, R. Diestel, and M. Kriesell, Global connectivity and expansion: long cycles and factors in $f$-connected graphs, Combinatorica 26 (2006), no. 1, 17-36.

11. R. A. Brualdi and H. J. Ryser, Combinatorial matrix theory, Cambridge University Press, 1991.

12. P. A. Catlin, On the Hajnal-Szemerédi theorem on disjoint cliques, Utilitas Math. 17 (1980), 163-177. 
13. P. Châu, An Ore-type theorem on hamiltonian square cycles, Graph. Combinator. 29 (2013), no. 4, 795834 .

14. D. Christofides, J. Hladký, and A. Máthé, Hamilton cycles in dense vertex-transitive graphs, J. Combin. Theory Ser. B 109 (2014), 34-72.

15. D. Christofides, P. Keevash, D. Kühn, and D. Osthus, Finding Hamilton cycles in robustly expanding digraphs, J. Graph Algorithms Appl. 16 (2012), no. 2, 335-358.

16. V. Chvátal, On Hamilton's ideals, J. Combin. Theory Ser. B 12 (1972), no. 2, 163-168.

17. P. Condon, J. Kim, D. Kühn, and D. Osthus, A bandwidth theorem for approximate decompositions, Proc. London Math. Soc. 118 (2019), no. 6, 1393-1449.

18. M. Coulson and G. Perarnau, Rainbow matchings in Dirac bipartite graphs, arXiv:1711.02916 (2017).

19. L. DeBiasio, R. Martin, and T. Molla, Powers of Hamiltonian cycles in multipartite graphs, arXiv:2106.11223 (2021).

20. G. A. Dirac, Some theorems on abstract graphs, Proc. Lond. Math. Soc. 3 (1952), no. 1, 69-81.

21. D. P. Dubhashi and A. Panconesi, Concentration of measure for the analysis of randomized algorithms, Cambridge University Press, 2009.

22. O. Ebsen, G. Maesaka, Chr. Reiher, M. Schacht, and B. Schülke, Embedding spanning subgraphs in uniformly dense and inseparable graphs, Random Struct. Algorithms 57 (2020), no. 4, 1077-1096.

23. P. Erdös, P. Frankl, and V. Rödl, The asymptotic number of graphs not containing a fixed subgraph and a problem for hypergraphs having no exponent, Graph. Combinator. 2 (1986), no. 1, 113-121.

24. J. Fan, B. Jiang, and Q. Sun, Hoeffding's inequality for general Markov chains and its applications to statistical learning, J. Mach. Learn. Res. 22 (2021), no. 139, 1-35.

25. E. Fischer, Variants of the Hajnal-Szemerédi theorem, J. Graph Theory 31 (1999), no. 4, 275-282.

26. A. Freschi, J. Hyde, and A. Treglown, On deficiency problems for graphs, to appear in Comb. Probab. Comput. (2021).

27. F. Garbe, R. Lang, A. Lo, R. Mycroft, and N. Sanhueza-Matamala, Lehel's conjecture for 3-graphs, in preparation (2021).

28. D. Gillman, A Chernoff bound for random walks on expander graphs, SIAM J. Comput. 27 (1998), no. 4, 1203-1220.

29. S. Glock, S. Gould, F. Joos, D. Kühn, and D. Osthus, Counting Hamilton cycles in Dirac hypergraphs, Comb. Probab. Comput. 30 (2020), no. 4, 631-653.

30. S. Glock and F. Joos, A rainbow blow-up lemma, Random Struct. Algorithms 56 (2020), no. 4, 1031-1069.

31. R. J. Gould, Recent advances on the Hamiltonian problem: Survey III, Graph. Combinator. 30 (2014), no. $1,1-46$.

32. A. Hajnal and E. Szemerédi, Proof of a conjecture of P. Erdős, Combinatorial theory and its applications, II (Proc. Colloq., Balatonfüred, 1969), 1970, pp. 601-623.

33. D. Hefetz, M. Krivelevich, and T. Szabó, Hamilton cycles in highly connected and expanding graphs, Combinatorica 29 (2009), no. 5, 547-568.

34. J. Hyde, H. Liu, and A. Treglown, A degree sequence Komlós theorem, SIAM J. Discrete Math. 33 (2019), no. 4, 2041-2061.

35. J. Hyde and A. Treglown, A degree sequence version of the Kühn-Osthus tiling theorem, Electron. J. Combin. 27 (2020), no. 3, Paper No. 3.48, 30. MR 4245161

36. S. Janson, T. Łuczak, and A. Ruciński, Random Graphs, John Wiley \& Sons, 2000.

37. J. P. Jarvis and D. R. Shier, Graph-theoretic analysis of finite Markov chains, Applied mathematical modeling: a multidisciplinary approach (1999), 85.

38. P. Keevash and R. Mycroft, A geometric theory for hypergraph matching, vol. 233, Mem. Amer. Math. Soc., 2015.

39. _ A multipartite Hajnal-Szemerédi theorem, J. Combin. Theory Ser. B 114 (2015), 187-236.

40. H. A. Kierstead and A. V. Kostochka, An Ore-type theorem on equitable coloring, J. Combin. Theory Ser. B 98 (2008), no. 1, 226-234.

41. F. Knox and A. Treglown, Embedding spanning bipartite graphs of small bandwidth, Comb. Probab. Comput. 22 (2013), no. 1, 71-96.

42. J. Komlós, G. N. Sárközy, and E. Szemerédi, Blow-Up Lemma, Combinatorica 17 (1997), no. 1, 109-123.

43. _ An algorithmic version of the blow-up lemma, Random Struct. Algorithms 12 (1998), no. 3, $297-312$.

44. __ Proof of the Seymour conjecture for large graphs, Ann. Comb. 2 (1998), no. 1, 43-60.

45. J. Komlós and M. Simonovits, Szemerédi's Regularity Lemma and its applications in graph theory, Combinatorics, Paul Erdős is Eighty (D. Miklós, V. T. Sós, and T. Szőnyi, eds.), vol. 2, Bolyai Society Mathematical Studies, 1996, pp. 295-352.

46. D. Kühn, A. Lo, D. Osthus, and K. Staden, The robust component structure of dense regular graphs and applications, Proc. Lond. Math. Soc. (3) 110 (2015), no. 1, 19-56.

47. D. Kühn and D. Osthus, Embedding large subgraphs into dense graphs, Surveys in combinatorics, London Math. Soc. Lecture Note Ser., vol. 365, Cambridge Univ. Press, Cambridge, 2009, pp. 137-167. 
48. _ Hamilton decompositions of regular expanders: a proof of Kelly's conjecture for large tournaments, Adv. Math. 237 (2013), 62-146.

49. - Hamilton cycles in graphs and hypergraphs: an extremal perspective, Proc. ICM 2014 (Seoul, Korea), vol. 4, 2014, pp. 381-406.

50. D. Kühn, D. Osthus, and A. Taraz, Large planar subgraphs in dense graphs, J. Combin. Theory Ser. B 95 (2005), no. 2, 263-282.

51. D. Kühn, D. Osthus, and A. Treglown, An Ore-type theorem for perfect packings in graphs, SIAM J. Discrete Math. 23 (2009), no. 3, 1335-1355.

52. D. Kühn, D. Osthus, and A. Treglown, Hamiltonian degree sequences in digraphs, J. Combin. Theory Ser. B 100 (2010), no. 4, 367-380.

53. R. Lang and N. Sanhueza-Matamala, Minimum degree conditions for tight Hamilton cycles, to accepted J. Lond. Math. Soc. (2020).

54. S. Letzter, Monochromatic cycle partitions of 2-coloured graphs with minimum degree $3 n / 4$, Electron. J. Combin. 26 (2019), no. 1, Paper No. 1.19, 67.

55. A. Lo and K. Markström, A multipartite version of the Hajnal-Szemerédi theorem for graphs and hypergraphs, Combin. Probab. Comput. 22 (2013), no. 1, 97-111.

56. A. Lo and N. Sanhueza-Matamala, An asymptotic bound for the strong chromatic number, Combin. Probab. Comput. 28 (2019), no. 5, 768-776.

57. C. Magyar and R. Martin, Tripartite version of the Corrádi-Hajnal theorem, Discrete Math. 254 (2002), no. 1-3, 289-308.

58. R. Martin and E. Szemerédi, Quadripartite version of the Hajnal-Szemerédi theorem, Discrete Math. 308 (2008), no. 19, 4337-4360.

59. C. McDiarmid, On the method of bounded differences, Surveys in Combinatorics, 1989: Invited Papers at the Twelfth British Combinatorial Conference (Cambridge), Cambridge University Press, 1989, pp. 148188.

60. R. Montgomery, A. Pokrovskiy, and B. Sudakov, A proof of ringel's conjecture, arXiv:2001.02665 (2020).

61. J. Moon and L. Moser, On Hamiltonian bipartite graphs, Israel J. Math. 1 (1963), 163-165.

62. R. Nenadov, B. Sudakov, and A. Z. Wagner, Completion and deficiency problems, J. Combin. Theory Ser. B 145 (2020), 214-240.

63. O. Ore, Note on Hamilton circuits, Amer. Math. Monthly 67 (1960), 55.

64. L. Pósa, A theorem concerning Hamilton lines, Magyar Tud. Akad. Mat. Kutató Int. Közl 7 (1962), 225-226.

65. S. Rao, A Hoeffding inequality for Markov chains, Electron. Commun. Probab. 24 (2019), 11 pp.

66. A. Schrijver, Combinatorial Optimization: Polyhedra and Efficiency, vol. 24, Springer Science \& Business Media, 2003.

67. B. Schülke, A pair-degree condition for Hamiltonian cycles in 3-uniform hypergraphs, arXiv:1910.02691 (2019).

68. K. Staden and A. Treglown, On degree sequences forcing the square of a hamilton cycle, SIAM J. Discrete Math. 31 (2017), no. 1, 383-437.

69. — The bandwidth theorem for locally dense graphs, Forum Math. Sigma 8 (2020), p42.

70. E. Szemerédi, Regular partitions of graphs, Colloq. Internat. CNRS 260 (1976), 399-401.

71. A. Treglown, A degree sequence Hajnal-Szemerédi theorem, J. Combin. Theory Ser. B 118 (2016), 13-43. 72 . , personal communication, 2020.

\section{Appendix A. Constructions}

In this section, we discuss a few constructions that in one way or the other suggest that our results and definitions are close to certain obstacles.

A.1. Pósa-type conditions. In this subsection we show that the degree conditions of Theorem 2.6 (Bandwidth theorem under Pósa-type conditions) are almost tight.

Proposition A.1. Let $k \geqslant 3$ and let $n$ be sufficiently large and divisible by $k$. Then there exists a graph $G$ on $n$ vertices whose degree sequence $d_{1} \leqslant \cdots \leqslant d_{n}$ satisfies $d_{i} \geqslant \frac{k-2}{k} n+i+$ $\frac{n^{1 / 2}}{12 k}$, for $i \leqslant n / k$. Moreover, $G$ does not contain the $(k-1)$ th power of a Hamilton cycle.

The proof of Proposition A.1 relies on the following construction of Balogh, Kostochka and Treglown [3] which covers the case of $k=3$.

Construction A.2. Let $n$ be divisible by 3 , and let $c_{n}=\left\lfloor n^{1 / 2} / 12\right\rfloor$ and $k_{n}=\left\lceil n^{1 / 2}\right\rceil$. Let $B_{n}$ be the graph on $n$ vertices consisting of three vertex classes $V_{1}=\{v\}, V_{2}$ and $V_{3}$ where $\left|V_{2}\right|=n / 3+c_{n}+1$ and $\left|V_{3}\right|=2 n / 3-c_{n}-2$, and has the following edges:

(i) all edges from $v$ to $V_{2}$, 
(ii) all edges between $V_{2}$ and $V_{3}$, and all possible edges inside $V_{3}$,

(iii) $k_{n}$ vertex-disjoint stars in $V_{2}$, each of size $\left\lfloor\left|V_{2}\right| / k_{n}\right\rfloor$ or $\left\lceil\left|V_{2}\right| / k_{n}\right\rceil$, which cover all of $V_{2}$.

Proposition A.3 ([3, Proposition 22]). For $n$ sufficiently large, let $B=B_{n}$ and denote its degree sequence by $d_{1} \leqslant \cdots \leqslant d_{n}$. Then

(1) $d_{i} \geqslant n / 3+c_{n}+i$ for all $1 \leqslant i \leqslant n / 3$,

(2) $B[N(v)]=V_{2}$ does not contain any path of length 3 .

A necessary condition for a graph $G$ to have the square of a Hamilton cycle is that, for every $x \in V(G), G[N(x)]$ contains a path of length 3. Thus Proposition A.3(2) shows that $B_{n}$ does not contain the square of a Hamilton cycle. We use the graphs $B_{n}$ to show Proposition A.1.

Proof of Proposition A.1. We can assume $k \geqslant 4$. Let $N=k n$ be divisible by $k$. Consider a complete $(k-2)$-partite graph with $k-3$ clusters of size exactly $n$ and one large cluster $U$ of size $3 n$. Inside the large cluster $U$, we place a copy of the graph $B_{3 n}$. Let $B$ be the resulting graph on $N$ vertices.

Note that every vertex outside of $U$ has degree exactly $(k-1) n=(k-1) N / k$, and each vertex in $U$ has exactly $(k-3) n=(k-3) N / k$ neighbours outside $U$. From Proposition A.3(1) it is straightforward to check that if $d_{1} \leqslant \cdots \leqslant d_{N}$ is the degree sequence of $B$, then $d_{i} \geqslant(k-2) N / k+c_{3 n}+i \geqslant(k-2) N / k+N^{1 / 2} /(12 k)+i$ for all $i \leqslant N / k$, as required.

It remains to show that $B$ does not contain the $(k-1)$ th power a Hamilton cycle. To this end, let $\left\{V_{1}, V_{2}, V_{3}\right\}$ be the partition of $U$ corresponding to the partition of $B_{3 n}$ where $V_{1}=\{v\}$. Observe that it suffices to show that $B[N(v)]$ does not contain the $(k-2)$ th power of a path on $2 k-2$ vertices. Let us assume that $P=\left(u_{1}, \ldots, u_{2 k-2}\right)$ is such a path and show how this results in a contradiction.

Since $B \backslash U$ is $(k-3)$-partite and $v$ has no neighbours in $V_{3}$, every $k$-clique containing $v$ must intersect $V_{2}$ in exactly two vertices. Thus, every $(k-1)$-clique in $B[N(v)]$ must intersect $V_{2}$ in exactly two vertices. Moreover, $k-1$ consecutive vertices in $P$ span a $(k-1)$ clique, and thus each consecutive $k-1$ vertices in $P$ must contain exactly two vertices in $V_{2}$. Let $a, b \in\{1, \ldots, k-1\}$ be such that $a<b$ and $u_{a}, u_{b} \in V_{2}$. This implies that $u_{a+k-1}, u_{b+k-1} \in V_{2}$, and further both $u_{b}, u_{a+k-1}$ and $u_{a+k-1}, u_{b+k-1}$ are neighbours in $B$ (since each of these pairs belong to a common $(k-1)$-clique of consecutive vertices of $P$ ). Thus $\left(u_{a}, u_{b}, u_{a+k-1}, u_{b+k-1}\right)$ is a path of length 3 in $V_{2}$. Since $B[U]$ is a copy of $B_{3 n}$, this contradicts Proposition A.3(2).

A.2. Ore- and Pósa-type conditions. It is an easy exercise to show that every graph satisfying the conditions of Ore's theorem also satisfies the conditions of Pósa's theorem. For $k \geqslant 3$, however, a graph satisfying the conditions of Theorem 2.4 (Bandwidth theorem under Ore-type conditions) does not necesarily satisfy the conditions of Theorem 2.6 (Bandwidth theorem under Pósa-type conditions), so these results do not imply each other. This is shown by the following construction.

Proposition A.4. For $k \geqslant 3$ and $1 / n \ll \mu \ll 1 / k$ there is graph $G$ on $n$ vertices such that

(i) for each $x y \notin E(G), \operatorname{deg}(x)+\operatorname{deg}(y) \geqslant 2 \frac{k-1}{k} n+\mu n$, but

(ii) there exists $i \leqslant n / k$ such that $d_{i}<\frac{k-2}{k} n+i$.

Proof. Let $\beta=5 \mu$ and $n$ sufficiently large. We also assume $\beta, \mu, n$ are chosen so that all numbers denoting cardinalities in what follows are integers. Partition a set of $n$ vertices into three sets $X_{0}, X_{1}, X_{2}$ of sizes $\left|X_{0}\right|=n /(2 k),\left|X_{1}\right|=3 n /(2 k)+\beta n$ and $\left|X_{2}\right|=n-\left|X_{0}\right|-\left|X_{1}\right|$. Form $G$ by adding every edge completely contained in $X_{0}, X_{1}$ or $X_{2}$ and add every edge between $X_{2}$ and its complement. Moreover, between $X_{0}$ and $X_{1}$ add a bipartite graph such that every vertex in $X_{0}$ is joined to exactly $d_{0}:=\beta n$ vertices in $X_{1}$, and every vertex in $X_{1}$ is joined to $d_{1}:=\beta n\left|X_{0}\right| /\left|X_{1}\right|$ vertices in $X_{0}$. Since $\beta \ll 1 / k$ we can assume $d_{1} \geqslant \beta n / 4$.

Every vertex in $X_{0}$ has degree $\left|X_{0}\right|-1+\left|X_{2}\right|+d_{0}=(k-2) n / k+n /(2 k)-1$. Every vertex in $X_{1}$ has degree $\left|X_{1}\right|-1+\left|X_{2}\right|+d_{1} \geqslant(k-1) n / k+n /(2 k)+\beta n / 4-1$. Every vertex 
in $X_{2}$ has degree $n-1$. Thus the degree sequence consists of the vertices of $X_{0}, X_{1}, X_{2}$, in that order. Note that $\left|X_{0}\right| \leqslant n / k$ and the $\left|X_{0}\right|$ th vertex in the degree sequence has degree $(k-2) n / k+n /(2 k)-1<(k-2) n / k+\left|X_{0}\right|$, so item (ii) holds. On the other hand, the only non-edges consist of a pair of vertices $x y$ with $x \in X_{0}, y \in X_{1}$. For these pairs, we have

$$
\begin{aligned}
\operatorname{deg}(x)+\operatorname{deg}(y) & =\left|X_{0}\right|-1+\left|X_{2}\right|+d_{0}+\left|X_{1}\right|-1+\left|X_{2}\right|+d_{1} \\
& \geqslant 2 \frac{k-1}{k} n-2+\frac{\beta n}{4} \geqslant 2 \frac{k-1}{k} n+\mu n,
\end{aligned}
$$

so item (i) holds, as required.

A.3. Robustly having a Hamilton framework does not imply Hamiltonicity. In the following we construct a graph $G$ in which every approximation $G^{\prime} \subseteq G$ admits some (spanning) 3-uniform Hamilton framework $\left(G^{\prime}, H^{\prime}\right)$, but which does not contain a square of a Hamilton cycle. This explains why we require the Hamilton framework itself to be robust, as opposed to robustly containing a Hamilton framework.

Let $q$ be some large prime, and let $n=4 q$. Let $P_{1}$ and $P_{2}$ be cubes of two paths on vertex sets $v_{1}, \ldots, v_{n}$ and $v_{n+1}, \ldots, v_{2 n}$ where the path ordering follows the labels. Let $C$ be a cube of a cycle on $v_{1}, \ldots, v_{2 n}$ and vertex sequence $\left(v_{\sigma(1)}, \ldots, v_{\sigma(n)}\right)$ where $\sigma(i) \equiv 5 i \bmod 2 n$. (Note that $\sigma$ is a bijection, since 5 is coprime to $2 n$.) Let $F$ be a graph obtained from $P_{1} \cup P_{2} \cup C$ by adding a vertex $v_{0}$ and connecting it to $v_{1}, v_{2}, v_{n+1}, v_{n+2}$. Let $G$ be obtained from $F$ by blowing up the vertices $v_{1}, \ldots, v_{2 n}$ by a set $V_{i}$ of size $m$ each, where $m$ is sufficiently large. Let $\mathcal{H}=K_{3}(G)$ and note that $\mathcal{H}$ has two 3-uniform tight components corresponding to $P_{1}, P_{2}, v$ and $C$ respectively. However, $G$ does not contain the square of a Hamilton cycle, since the vertex $v_{0}$ is not on a square of a cycle.

To conclude, we show that $(G, \mathcal{H})$ has the property that any $(\mu, \mu)$-approximation $G^{\prime}$ of $G$ contains a 3-uniform zero-free Hamilton framework, provided that $1 / M \ll \mu \ll 1$. Indeed, let $\mathcal{H}^{\prime}=\mathcal{H} \cap K_{3}\left(G^{\prime}\right)$, and denote by $V_{i}^{\prime}$ the remainder of the cluster $V_{i}$ in $G^{\prime}$. Now, if $v_{1} \in V\left(G^{\prime}\right)$, then there is a spanning tight component $\mathcal{H}_{1} \subseteq \mathcal{H}^{\prime}$ with edges corresponding to the triangles of $P_{1}, P_{2}, v$. On the other hand, if $v_{1} \notin V\left(G^{\prime}\right)$, then there is a spanning tight component $\mathcal{H}_{2} \subseteq \mathcal{H}^{\prime}$ whose edges correspond to the (remaining) triangles of $C$. Since $P_{1}$ and $C$ are cubes of paths and cycles respectively, both $\mathcal{H}_{1}$ and $\mathcal{H}_{2}$ contain a tetrahedron. Moreover, it is also not hard to see that $\mathcal{H}_{1}$ and $\mathcal{H}_{2}$ have a perfect fractional matchings each. Indeed, in the case of $\mathcal{H}_{1}$ we begin by matching $v_{0}$ arbitrarily on a triangle with ends in $V_{1}^{\prime}$, $V_{2}^{\prime}$ and delete those endpoints from these clusters. Afterwards, we may find perfect fractional matchings in each $\mathcal{H}^{\prime}\left[V_{4 i+1}^{\prime} \cup \cdots \cup V_{4 i+4}^{\prime}\right]$ for $0 \leqslant i \leqslant q-1$, which is possibly by Theorem 2.11 and $1 / M \ll \mu \ll 1$. Repeating the same argument for $P_{2}$ gives a perfect fractional matching of $\mathcal{H}_{1}$. Similarly, we see that $\mathcal{H}_{2}$ has a perfect fractional matching, by first partitioning each $V_{i}^{\prime}$ into almost balanced clusters $V_{i}^{1}, \ldots, V_{i}^{4}$ and then finding perfect fractional matchings in each $\mathcal{H}^{\prime}\left[V_{\sigma(i+1)}^{1} \cup V_{\sigma(i+2)}^{2} \cup V_{\sigma(i+3)}^{3} \cup V_{\sigma(i+4)}^{4}\right]$ for $0 \leqslant i \leqslant 2 n-1$. Hence $G^{\prime}$ does indeed contain a zero-free Hamilton framework.

\section{APPENDIX B. REMARKS ON FRAMEWORKS}

In this appendix we give more details about the remarks on Hamilton frameworks made in Section 3.5.

B.1. Searching for Hamilton frameworks. In the following, we argue that one can search for a $k$-uniform Hamilton framework in a given graph $G$ on $n$ vertices in time $O\left(n^{7 k}\right)$. We did not make a serious effort to optimise the number in the exponent.

We begin by identifying the tight components of $K_{k}(G)$. Let $D$ be the directed graph whose vertices are the ordered $k$-cliques of $G$ and a directed edge $K_{1} K_{2}$ whenever the last $k-1$ vertices of $K_{1}$ coincide with the first $k-1$ vertices of $K_{2}$. Note that $|V(D)|=O\left(n^{k}\right)$ and $|E(D)|=O\left(n^{k+1}\right)$. Recall that two vertices in a directed graph are in the same strong connectivity component if they are on a common closed directed walk. Observe that each tight component of $K_{k}(G)$ corresponds to at most $k$ ! strong connectivity components in 
$G$ (corresponding to all possible reorderings of an edge). This allows us to find all tight components of $G$ by identifying the strongly connected components of $D$, using e.g. Tarjan's algorithm, which runs in time $O(|V(D)|+|E(D)|)=O\left(n^{k+1}\right)$.

Now consider a tight component $C \subseteq K_{k}(G)$. Since $K_{k}(G)$ has at most $n^{k}$ tight components, it suffices to show that one can check in time $O\left(n^{6 k}\right)$ whether $C$ admits a perfect fractional matching and contains a closed tight walk of length coprime to $k$. Finding a perfect fractional matching can be phrased as solving an instance of linear programming with $n^{k}$ variables (one for each edge) and $n$ restrictions (one for each vertex). This can be done in time $O\left(n^{6 k}\right)$ using interior-point methods [66]. Turning to aperiodicity, let $D^{\prime}$ be the directed graph associated with $C$ in the same way as above. Observe that a tight closed walk of length $\ell$ in $C$ corresponds to a closed directed walk of length $\ell$ in $D^{\prime}$ and vice versa. Let $d$ be the greatest common divisor of all directed cycle lengths of $D^{\prime}$. Note that $d$ is also a common divisor of the lengths of all closed directed walks in $D^{\prime}$. Using a depth-first search in $D^{\prime}$, one can determine $d$ in $O\left(\left|V\left(D^{\prime}\right)\right|+\left|E\left(D^{\prime}\right)\right|\right)=O\left(n^{k+1}\right)$ steps [37]. Hence, it is decidable in time $O\left(n^{k+1}\right)$ whether $C$ contains a closed tight walk of length coprime to $k$. Moreover, the same algorithm also finds such a walk when $d$ is coprime to $k$.

B.2. Linked edges. This section is dedicated to the proof of Proposition 3.6. We shall use the following consequence of the Graph Removal Lemma [23]. For every $\varepsilon>0$ and $k \in \mathbb{N}$ there exists $\delta>0$ and $n_{0} \in \mathbb{N}$ such that for all graphs $G$ on $n \geqslant n_{0}$ vertices the following holds: if one needs to delete more than $\varepsilon n^{2}$ edges to eliminate all $k$-cliques of $G$, then $G$ contains at least $\delta n^{k}$ many $k$-cliques.

Proof of Proposition 3.6. Clearly, we only need to show that there exists $\mu^{\prime} \leqslant \mu$ such that every vertex has at least $\mu^{\prime} n^{k}$ linked edges in $K_{k}(G)$. Let $v \in V(G)$ be an arbitrary vertex. Let $G_{v} \subseteq G-v$ be the subgraph formed by all the edges which intersect $N_{G}(v)$. Note that each edge in $K_{k}\left(G_{v}\right)$ intersects $N_{G}(v)$ in at least $k-1$ vertices and does not contain $v$, and thus corresponds to a linked edge of $v$ in $K_{k}(G)$. It is therefore enough to show that $G_{v}$ contains at least $\mu^{\prime} n^{k}$ many $k$-cliques.

We want to show that, after the removal of any $\left(\mu^{2} / 8\right) n^{2}$ edges from $G_{v}$, at least one $k$-clique remains in $G_{v}$. Indeed, suppose we remove $\left(\mu^{2} / 8\right) n^{2}$ edges $Y$ from $G_{v}$. A doublecounting reveals that at most $(\mu / 2) n$ vertices had more than $(\mu / 2) n$ edges removed from them, let $X$ be those vertices. Note that, in particular since $v \notin V\left(G_{v}\right), v \notin X$. In $G$, remove the edges corresponding to $Y$ and also remove the vertices corresponding to $X$, to obtain a subgraph $G^{\prime} \subseteq G$. Note that $v \in V\left(G^{\prime}\right)$. Clearly, $G^{\prime}$ is a $\mu$-approximation of $G$. Since $\left(G, K_{k}(G)\right)$ is a $\mu$-proto-robust Hamilton framework, we deduce $K_{k}\left(G^{\prime}\right)$ is a Hamilton framework. We note that $v \in V\left(G^{\prime}\right)$. Since $K_{k}\left(G^{\prime}\right)$ is a Hamilton framework, it contains a perfect fractional matching. In particular, there is at least one edge $e_{1}$ in $K_{k}\left(G^{\prime}\right)$ containing $v$. Since $v\left(G^{\prime}\right) \geqslant 2 k$, there must also exist an edge $e_{2}$ not containing $v$. Since $K_{k}\left(G^{\prime}\right)$ is tightly connected, there must exist a tight walk between $e_{1}, e_{2}$, and the first edge of that walk not containing $v$ is a linked edge of $v$ in $K_{k}\left(G^{\prime}\right)$. By construction, this corresponds to a $k$-clique in $G_{v}-Y$, as required.

Assuming $n$ is sufficiently large, what we have shown implies that $G_{v}$ satisfies the assumptions of the Graph Removal Lemma [23]. Thus there exist $\mu^{\prime}>0$ such that the number of $k$-cliques in $G_{v}$ is at least $\mu^{\prime} n^{k}$, as desired.

\section{Appendix C. Structural tools}

In this section, we give proofs of a few structural tools used in Section 7 that we have borrowed from our joint work with Garbe, Lo and Mycroft [27].

C.1. Allocation. In this section, we show Proposition 7.6.

Proof of Proposition 7.6. We will consider an integer flow $f$ on $\hat{\mathcal{H}}$. This means that $f$ assigns integers to the directed edges of $\hat{\mathcal{H}}$, such that $f\left(x, x^{\prime}\right)=-f\left(x^{\prime}, x\right)$ for every edge $x x^{\prime} \in E(\hat{\mathcal{H}})$. Consider a part $U \in \mathcal{U}$. Since $\hat{\mathcal{H}}[U]$ is connected and $\sum_{v \in U} \mathbf{b}(v)=0$, we can choose an integer 
flow $f$ such that each vertex $x \in U$ receives flow $\sum_{x x^{\prime} \in \hat{\mathcal{H}}[U]} f\left(x^{\prime}, x\right)=\mathbf{b}(x)$. Indeed, we can construct such a flow in an iterative fashion, starting from an empty flow. If we are not done with our current flow $f$, then there must exist distinct vertices $x$ and $y$ in $U$ such that $0 \leqslant \sum_{x x^{\prime} \in E(\hat{\mathcal{H}}[U])} f\left(x^{\prime}, x\right)<\mathbf{b}(x)$ and $\mathbf{b}(y)<\sum_{y y^{\prime} \in E(\hat{\mathcal{H}}[U])} f\left(y^{\prime}, y\right) \leqslant 0$. We can send a unit flow from $y$ to $x$ along a path joining those vertices, by changing the flow in the edges of the path by one. Clearly, this iteration takes at most $\sum_{v \in U}|\mathbf{b}(v)| \leqslant s n$ steps. Each step changes the flow of an edge at most by one, thus we have that in the final flow, each $x x^{\prime} \in E(\hat{\mathcal{H}}[U])$ satisfies $\left|f\left(x, x^{\prime}\right)\right| \leqslant s n$. Repeating this for the others parts of $\mathcal{U}$ yields the desired flow.

We now define $\mathbf{w} \in \mathbb{Z}^{E(\mathcal{H})}$ as follows. Initially, we set $\mathbf{w}=0$. Consider each $x x^{\prime} \in E(\hat{\mathcal{H}})$ in turns. Suppose there is a positive flow $c$ from $x$ to $x^{\prime}$, that is $f\left(x, x^{\prime}\right)=c \geqslant 0$. Pick $Q_{x x^{\prime}} \in L_{\mathcal{H}}(x) \cap L_{\mathcal{H}}\left(x^{\prime}\right)$. We decrease the edge weight $\mathbf{w}\left(\{x\} \cup Q_{x x^{\prime}}\right)$ by $c$ and increase the edge weight $\mathbf{w}\left(\left\{x^{\prime}\right\} \cup Q_{x x^{\prime}}\right)$ by $c$.

The resulting $\mathbf{w}$ satisfies $\mathbf{b}(v)=\sum_{v \ni e} \mathbf{w}(e)$ for all $v \in V(\mathcal{H})$ by our construction. Note that for each edge $e \in E(\mathcal{H})$ there are at most $k n$ edges $x x^{\prime} \in E(\hat{\mathcal{H}})$ such that $e=\{x\} \cup Q_{x x^{\prime}}$ (indeed, there are $k$ choices for $x \in e$ and at most $n$ choices for $x^{\prime} \notin e$ ). Since each $x x^{\prime}$ has flow of size at most $s n$, we get $\|\mathbf{w}\|_{\infty} \leqslant k s n^{2}$.

C.2. Fractional matchings and bounded degree covers. Here we prove Lemma 7.7 (Bounded degree cover). To do so, we require a few definitions. Let $k \in \mathbb{N}$. We say that a hypergraph $\mathcal{G}$ is a $[k]$-graph if $|e| \in[k]$ for every edge $e \in E(\mathcal{G})$. We use the following non-standard form of restriction for $[k]$-graphs. Let $\mathcal{G}$ be a $[k]$-graph and let $X \subseteq V(\mathcal{G})$. Then $\mathcal{G} \cap X$ is the $[k]$-graph with vertex set $X$ and edge set $E(\mathcal{G} \cap X):=\{e \cap X: e \in E(\mathcal{G})\}$. For convenience, we write in the following $e \in \mathcal{G}$ instead of $e \in E(\mathcal{G})$ and $\mathbb{R}^{\mathcal{G}}$ instead of $\mathbb{R}^{E(\mathcal{G})}$ if the context is clear.

The following proposition follows immediately from these definitions.

Proposition C.1. Let $\mathcal{G}$ be $a[k]$-graph which admits a perfect fractional matching $\mathbf{w}$ and let $X \subseteq V(\mathcal{G})$. Then $\mathbf{w}^{\prime} \in \mathbb{R}^{\mathcal{G} \cap X}$ defined by $\mathbf{w}^{\prime}\left(e^{\prime}\right)=\sum_{e \in \mathcal{G}}: e \cap X=e^{\prime} \mathbf{w}(e)$ for each edge $e^{\prime} \in \mathcal{G} \cap X$ yields a perfect fractional matching $\mathbf{w}^{\prime}$ in $\mathcal{G} \cap X$.

Proposition C.2. Let $\mathcal{G}$ be a $[k]$-graph on $n$ vertices which admits a perfect fractional matching $\mathbf{w}$. Then for any set $X \subseteq V(\mathcal{G})$ with $|X|<n / k$, there is a non-empty edge of $\mathcal{G}$ which does not intersect $X$.

Proof. Since $\sum_{e \in \mathcal{G}}: v \in e=\mathbf{w}(e)=1$ for every $v \in V(\mathcal{G})$, we have $\sum_{e \in \mathcal{G}: e \cap X \neq \emptyset} \mathbf{w}(e) \leqslant|X|$ and $\sum_{e \in \mathcal{G}}|e| \mathbf{w}(e)=n$. It follows from the latter that $\sum_{e \in \mathcal{G}: e \neq \emptyset} \mathbf{w}(e) \geqslant n / k>|X|$, and so there must be some non-empty edge of $\mathcal{G}$ which does not intersect $X$.

Proposition C.3. Let $\mathcal{G}$ be a $[k]$-graph on $n$ vertices which admits a perfect fractional matching $\mathbf{w}$. Then there exists a perfect fractional matching $\mathbf{w}^{\prime}$ in $\mathcal{G}$ in which at most $n$ edges have non-zero weight.

Proof. Let $P=\left\{A_{\mathcal{G}} \mathbf{1}_{e}: e \in \mathcal{G}\right\} \subseteq \mathbb{R}^{V(\mathcal{G})}$ be the incidence matrix of $\mathcal{G}$. Since $\mathcal{G}$ admits a perfect fractional matching, the all-ones vector $\mathbf{1} \in \mathbb{R}^{V(\mathcal{G})}$ lies in the positive cone generated by the vectors of $P$, i.e. it can be written as a positive combination of those vectors. By the conic version of Carathéodory's theorem (see, e.g., [5, Section 1.2, Proposition 1.2.1]), 1 can be written as a positive combination of at most $n$ vectors in $P$, say $\mathbf{1}=\sum_{i \in[n]} \lambda_{i} A_{\mathcal{G}} \mathbf{1}_{e_{i}}$ for some $\lambda_{i} \geqslant 0$ and $e_{i} \in \mathcal{G}$. It follows immediately that $\lambda_{i} \leqslant 1$ for all $i \in[n]$. The proof is complete by setting $\mathbf{w}^{\prime}=\sum_{i \in[n]} \lambda_{i} \mathbf{1}_{e_{i}}$.

Proof of Lemma 7.7. Fix $k$. Suppose for a contradiction that the theorem does not hold, and let $\mathcal{G}$ be a counterexample of minimal order. So $\mathcal{G}$ is a $[k]$-graph on $n$ vertices which admits a perfect fractional matching $\mathbf{w}$ but does not admit a $\left(k^{2}+1\right)$-cover. By Proposition C.3, we may assume that at most $n$ edges of $\mathcal{G}$ have non-zero weight in $\mathbf{w}$. So we may assume that $n>k^{2}+1$. Let $\mathcal{H}$ be the spanning subgraph of $\mathcal{G}$ consisting of all such edges, namely $\mathcal{H}=\{e \in \mathcal{G}: \mathbf{w}(e) \neq 0\}$. Define $Y \subseteq V(\mathcal{H})$ to consist of all vertices of $\mathcal{H}$ with degree at least 
$k^{2}+1$ in $\mathcal{H}$. Then $\left(k^{2}+1\right)|Y| \leqslant k|\mathcal{H}| \leqslant k n$, so $|Y|<n / k$. By Proposition C.2, it follows that there is a non-empty edge $e^{*} \in \mathcal{H}$ which does not intersect $Y$; choose such an edge and set $X:=V(\mathcal{H}) \backslash e^{*}$. By Proposition C.1, there exists a perfect fractional matching $\mathbf{w}^{\prime}$ in $\mathcal{H} \cap X$, and since $\mathcal{G}$ was a minimal counterexample, it follows that $\mathcal{H} \cap X$ admits a $\left(k^{2}+1\right)$-cover $\mathcal{C}^{\prime}$. We now form a $\left(k^{2}+1\right)$-cover $\mathcal{C}$ in $\mathcal{H}$ as follows. For each edge $e \in \mathcal{C}^{\prime}$ choose an edge $f(e) \in \mathcal{H}$ with $e \subseteq f(e)$ and $f(e) \cap X=e$ (such an edge $f(e)$ must exist by definition of $\mathcal{H} \cap X$ ), and set $\mathcal{C}:=\left\{f(e): e \in \mathcal{C}^{\prime}\right\} \cup\left\{e^{*}\right\}$. Then each vertex of $X$ is contained in the same number of edges in $\mathcal{C}$ as in $\mathcal{C}^{\prime}$ (that is, between 1 and $k^{2}+1$ ). Furthermore, each vertex of $e^{*}$ is contained in at least one edge of $\mathcal{C}$ (namely $e^{*}$ ) and at most $k^{2}$ edges of $\mathcal{C}$ (since $e^{*}$ does not intersect $Y$, so each vertex of $e^{*}$ has degree at most $k^{2}$ in $\mathcal{H}$, and $\mathcal{C} \subseteq \mathcal{H}$ ). We conclude that $\mathcal{C}$ is a $\left(k^{2}+1\right)$-cover of $\mathcal{H}$ and therefore of $\mathcal{G}$, as required.

(R. Lang) Universität Heidelberg, Institut für Informatik, Im Neuenheimer Feld 205, 69120 Heidelberg, Germany

Email address: lang@informatik.uni-heidelberg.de

(N. Sanhueza-Matamala) Departamento de Ingeniería Matemática, Facultad de Ciencias Físicas y Matemáticas, Universidad de Concepción, Chile

Email address: nicolas@sanhueza.net 